$T 10 \cdots 25443$
$u C-80$

\title{
ANALYTICAL AND EXPERIMENTAL STUDIES RELATING TO THE SIMULATED START-UP OF IN-CORE THERMIONIC REACTOR SYSTEMS
}

\author{
by \\ James Garfield Guppy
}

JUNE 1970
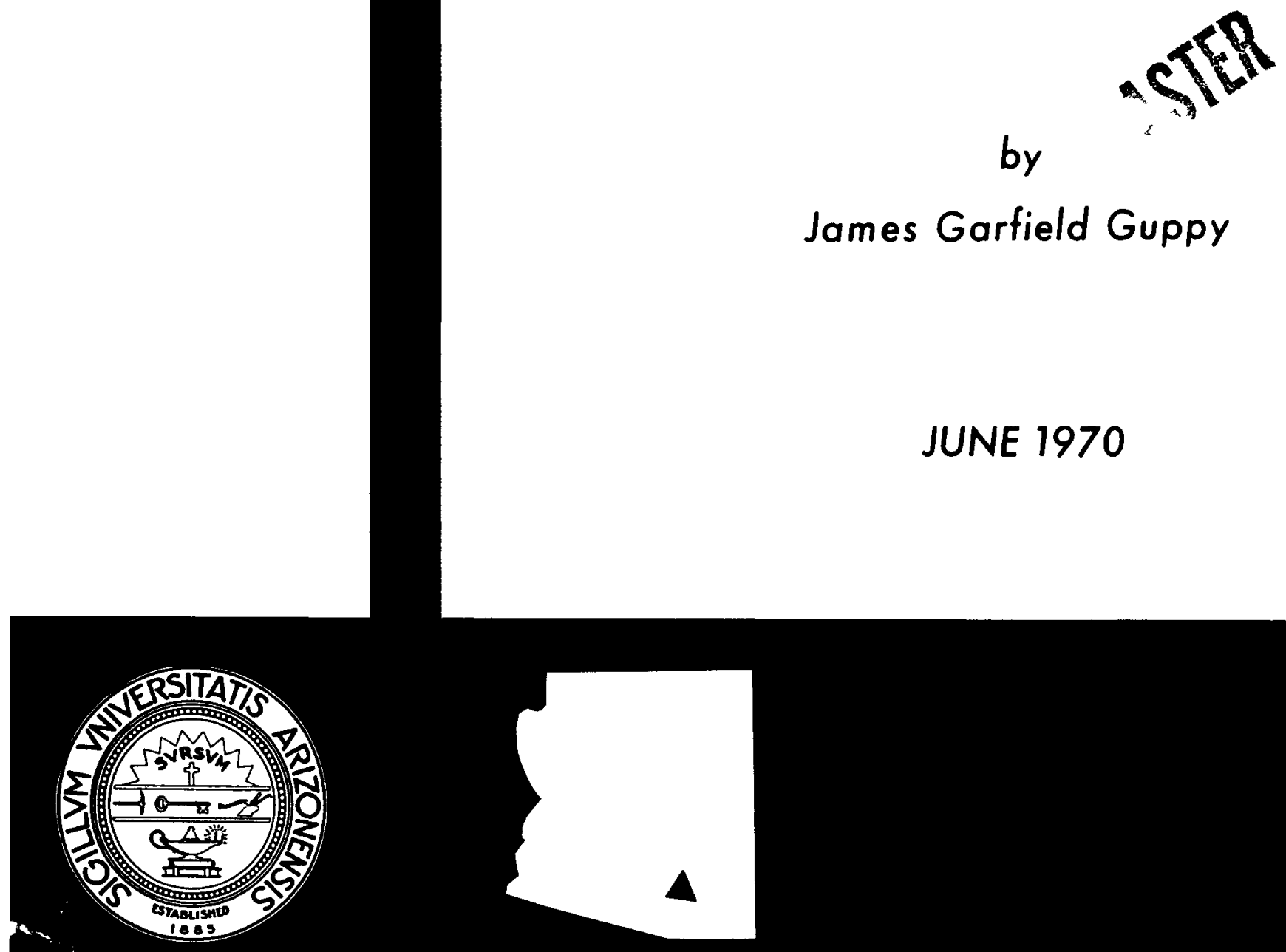


\section{DISCLAIMER}

This report was prepared as an account of work sponsored by an agency of the United States Government. Neither the United States Government nor any agency Thereof, nor any of their employees, makes any warranty, express or implied, or assumes any legal liability or responsibility for the accuracy, completeness, or usefulness of any information, apparatus, product, or process disclosed, or represents that its use would not infringe privately owned rights. Reference herein to any specific commercial product, process, or service by trade name, trademark, manufacturer, or otherwise does not necessarily constitute or imply its endorsement, recommendation, or favoring by the United States Government or any agency thereof. The views and opinions of authors expressed herein do not necessarily state or reflect those of the United States Government or any agency thereof. 


\section{DISCLAIMER}

Portions of this document may be illegible in electronic image products. Images are produced from the best available original document. 
ANALYTICAL AND EXPERIMENTAL STUDIES RELATING TO

THE SIMULATED START-UP OF IN-CORE THERMIONIC REACTOR SYSTEMS

by

James Garfield Guppy

Department of Nuclear Engineering
The University of Arizona
Tucson, Arizona 85721

June 1970

A dissertation submitted to the faculty of the Department of Nuclear Engineering in partial fulfillment of the requirements for the degree of Doctor of Philosophy in the Graduate College of the University of Arizona. Supported by the U. S. Atomic Energy Commission under Contract AT- $(04-3)-670$. 


\section{ACKNOWLEDGMENTS}

The author wishes to express his appreciation to

Dr. Richard L. Brehm for his advice and assistance during the performance of this research.

The author is indebted to the United States Atomic Energy Commission for providing financial support for this study under Contract AT-(04-3) -670 .

The author is grateful to Henrik G. Gronroos and Jerry P. Davis for their assistance and to the Jet Propulsion Laboratory for providing the opportunity to use certain facilities during the course of this work.

The University of Arizona Computer Center is acknowledged for the generous grant of computer time made available for this research. 
TABLE OF CONTENTS

Page

LIST OF ILLUSTRATIONS • . • • • • • • . . . . vii

LIST OF TABLES • • • • • • • • • • • • • •

ABSTRACT • • • • • • • • • • • • • •

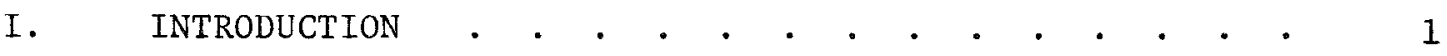

Purpose . . . . . . . . . . . . . . . 1

System Simulation Philosophy . . . . . . . 4

Content and Organization . . . . . . . . . 5

II. REACTOR SYSTEM ANALYSIS • • • • • • • • • • •

Development of a System Model •

The Single Diode Thermionic Reactor
Representation . . . . . . . . 10

Reactor Kinetics Description . . . . 12

Transient Thermal Equations . . . . 15

Solution Methodology . . . . . . . . 30

Comparison Between Analog and Digital Approaches . 30

A Simplified Model of Converter Energy
Transport . . . . . . . . . . . 31

Step in Power. . . . . . . . . . . .

Change in Load . . . . . . . . . . . 41

Discussion of Results . . . . . . . . 44

III. THERMIONIC ANALYSIS . . . . . . . . . . . . . 45

Basic Thermionic Operation . . . . . . . . 46

Development of Converter Analysis . . . . . . 49

Plasma Description . . . . . . . . . 50

Solution of Boltzmann Equations . . . 52

Solution of Macroscopic Equations . . 53

Sheath Analysis . . . . . . . . . . 58

Surface Analysis . . . . . . . . . . 60

Work Function Determination . . . . 60

Schottky Effects • • . . . . . . 62

Numerical Method of Solution . . . . . $\cdot 63$

Extension to Transient Analysis • • . • • • 66

Inclusion of the Load Line . . . . . . 68 
TABLE OF CONTENTS-Continued.

Page

Enhancement of Convergence . . . . . . 69

Specific Modifications Incorporated . . 69

Convergence Criteria Used . . . . . 72

Use of the Transient Thermionic Analysis . . . 72

Specification of Parameters . . . . . 72

Frequency of Usage • . . . • • . . . . 73

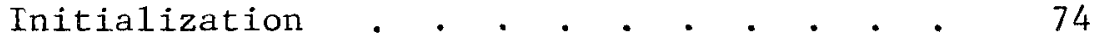

Comparison to Richardson-Dushnan Approach • . • 76

Static Comparisons . . . . . . . . . 76

Transient Comparisons . . • . . . . • 82

Positive Step in Power . . . . . . 82

Change in Load . . . . . . . . . 83

Discussion of Results . . . . . . . . 83

IV. EXPERIMENTAL STUDIES • • • • • • • • • • •

Experimental Arrangement . . . . . . . . . 90

Analytical Model . . . . . . . . . . . 92

Comparison Studies. . . . . . . . . . . 97

Steady State Analysis . . . . . . . . 97

Transient Analysis . . . . . . . . . 103

Three Parallel Diode Model . . . . 103

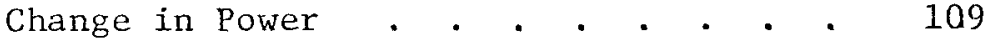

Change in Load. . . . . . . . . 112

Thermionic Burnout . . . . . . . 115

Discussion of Results . . . . . . . . 120

V. SIMULATED REACTOR SYSTEM START-UP • • • • • • . 122

Reactor System Description . . . . . . • . 122

Start-up Cases . . . . . . . . . . . . 124

Constant Output Voltage Start-up . . . . 127

Constant Emitter Temperature Start-up . . . 131

Discussion of Results . . . . . . . . . . 135

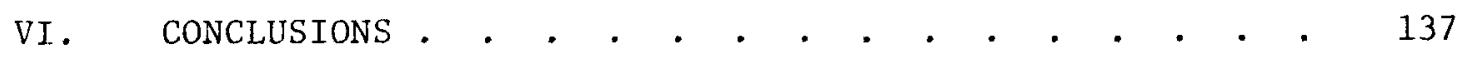

APPENDIX: SYSTEM DYNAMICS PROGRAM . • . • . . . 140

LIST OF REFERENCES • • • • • • • • • • • • 190 


\section{LIST OF ILLUSTRATIONS}

1.1. Overall Thermionic Reactor System . . . . . . .

2.1. Typical Representation of Series-Connected In-core Diode Assembly for Flashlight Configuration . . . . 11

2.2. Representation of Regions for Single Diode Approximation . . . . . . . . . . . . . .

2.3. Reactor System Diagram Showing Location of Coolant Loop Temperatures . . . . . . . . . . . . 23

2.4. "Uphil1" Mode Voltage Profile for R-D Analysis . • . 36

2.5. Comparison of Nonlinear Systen Response for +2 c Step . 38

2.6. Comparisons of Nonlinear and Linear System Responses for +2 c Step . . . . . . . . . . . . . .

2.7. Comparison of Nonlinear System Response for Change in Load . . . . . . . . . . . . . . . .

2.8. Comparisons of Nonlinear and Linear System Responses for Change in Load . . . . . . . . . . . .

3.1. Basic Parameters and Processes Involved in Thermionic Converter Operation . . . . . . . . . . . . 47

3.2. Typical Voltage Profile Across Thermionic Diode • • . 59

3.3. Relation of Surface and Plasma Current Densities . . . 61

3.4. Typical Steady-State I-V Curve with Superimposed Load Line. . . . . . . . . . . . . . .

3.5. Steady-State Comparisons of R-D and Present Thermionic

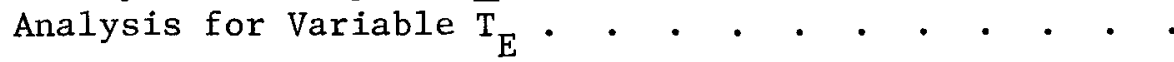

3.6. Steady-State Comparisons of $R-D$ and Present Thermionic Analysis for Variable $\overline{\mathrm{T}}_{\mathrm{C}} \cdot \cdot \cdot \cdot \cdot \cdot \cdot \cdot \cdot \cdot \cdot \cdot \cdot \cdot \cdot$ 
LIST OF ILLUSTRATIONS---Continued

Figure

Page

3.7. Comparison of Present Thermionic Analysis to $R-D$

Approach for +2 c Step . . . . . . . . . . . .

3.8. Comparison of Present Thermionic Analysis to $R-D$ Approach for Change in Load . . . . . . . . . 85

4.1. Experimental Diode Assembly . . . . . . . . . . 91

4.2. Simulated Diode Assembly . . . . . . . . . . . 93

4.3. Steady-State Comparisons of Experimental and Analytical I-V Curves. . . . . . . . . . . . . . .

4.4. Three Parallel-Connected Diode Model . . . . . . .

4.5. Comparison of Three Parallel Diode Model and Experimental System Responses for + Power Ramp . . . . • . .

4.6. Comparison of Single Average Diode Model and Experimental System Responses for + Power Ramp . . . . . . . .

4.7. Comparison of Three Parallel Diode Model and Experimental System Responses for Decrease in Load . . . . . .

4.8. Comparison of Single Average Diode Model and Experimental System Responses for Decrease in Load . . . . . . .

4.9. Effect of Heat Input on Emitter Temperature at Constant Reservoir Temperature . . . . . . . . . . .

4.10. Comparison of Three Parallel Diode Model and Experimental System Responses for Thermionic Burnuut . . . . .

4.11. Comparison of Single Average Diode Model and Experimental System Responses for Thermionic Burnout . . . . .

5.1. Constant Voltage Start-up . . . . . . . . . . 130

5.2. Constant Emitter Temperature Start-up . . . . . . . 134

A.1. Program Structure for Transient System Analysis . • . 141 


\section{LIST OF TABLES}

Table

Page

2.1. Reactor System Equations . . . . . . . . . . . 29

2.2. Equilibrium Conditions and System Parameters for Reactor Model Comparisons . . . . . . . . . . . 32

2.3. Dynamic Equations for Reactor System Comparisons . . . 33

3.1. Ranges of Validity of Thermionic Analysis . . . . . 50

3.2. Reference Operating Conditions . . • . • . . . . 77

4.1. Parameters for Experimental Studies . . . . . . . 98

4.2. Dynamic Equations for Experimental Studies . . . . . 99

5.1. Ful1 Power Equilibrium Conditions and System Parameters for Start-up Studies . . . . . . . . . . . 125

5.2. Dynamic Equations for Start-up Studies . . . . . . 126

5.3. Initial Conditions for Constant Voltage Start-up • • . 129

5.4. Initial Conditions for Constant Emitter Temperature
Start-up . . . . . . . . . . . . . . 133 
ABSTRACT

An analytical representation of a nuclear reactor containing in-core thermionic devices suitable for transient studies is formulated. The resulting model is applicable to situations involving substantial changes in system operating conditions, as would be experienced during start-up transients.

Neutron kinetics and heat transfer are represented by "nodal" descriptions. Contributions from all system regions of importance are retained to produce realistic transient response. The resulting set of equations is coupled to a digital computer integration routine to solve for the dynamic response.

The thermionic converter physics is described by a complex iterative numerical scheme based on a diffusion approximation to the plasma processes. Other thermionic processes included are surface and Schottky effects, plus an accounting of the electrostatic sheaths present. The analysis is extended to include general application to thermionic diodes undergoing transients.

The digital description of the reactor model is tested against a comparable analog computer simulation and shown to yield better accuracy. The complex thermionic analysis is compared to a simpler converter physics description and found to be far superior in predicting the electrical characteristics of the converter for large changes in operating conditions. The thermionic analysis is also compared 
with transient experimental diode data over wide ranges of converter operations and shown to produce excellent agreement. Application of the model to system start-up is described for two postulated start-up approaches encompassing either constant diode voltage or constant emitter temperature.

This thermionic reactor model is shown to be very useful in obtaining insight and understanding into the overall system dynamic behavior during large changes in system operating conditions. Furthermore, since the thermionic analysis can be decoupled easily from the system model, separate application to studies involving only transient diode operations may be accomplished.

An important finding of these analytical studies is that under certain conditions, the results obtained assuming an average and uniform description of the temperature distributions, especially for the emitter surface, may not be sufficiently accurate to represent all of the important aspects of diode transient behavior. Analytical studies involving the complete reactor model demonstrate that simple control methods may be adequate to produce very reasonable response during system transients. 
CHAPTER I

INTRODUCTION

\section{Purpose}

The purpose of this study is to investigate the dynamic behavior, particularly during start-up, of a nuclear reactor utilizing in-core thermionic diodes. Such reactors have potential usage as power sources in future space missions. Thermionic conversion promises an inherent reliability over other electricity producing methods since the process requires no moving parts. However, the operation of a reactor containing such devices poses certain problems which are as yet unresolved.

A reactor core containing thermionic devices will necessarily combine a variety of technical problems into one piece of hardware. The high temperatures required for efficient converter operation produce material and compatability problems. They may also cause fuel swelling which affects diode spacing and could lead to electrical failures. Coolant activation by neutron absorption necessitates a twoloop heat rejection system so that radiator shielding is not required. The United States Atomic Energy Commission is presently considering construction of such a reactor, and one of the major goals of that project will be to check system stability and dynamic behavior.

A schematic of the contemplated reactor system is shown in Figure 1.1. Within the reactor, the thermionic diodes are stacked 


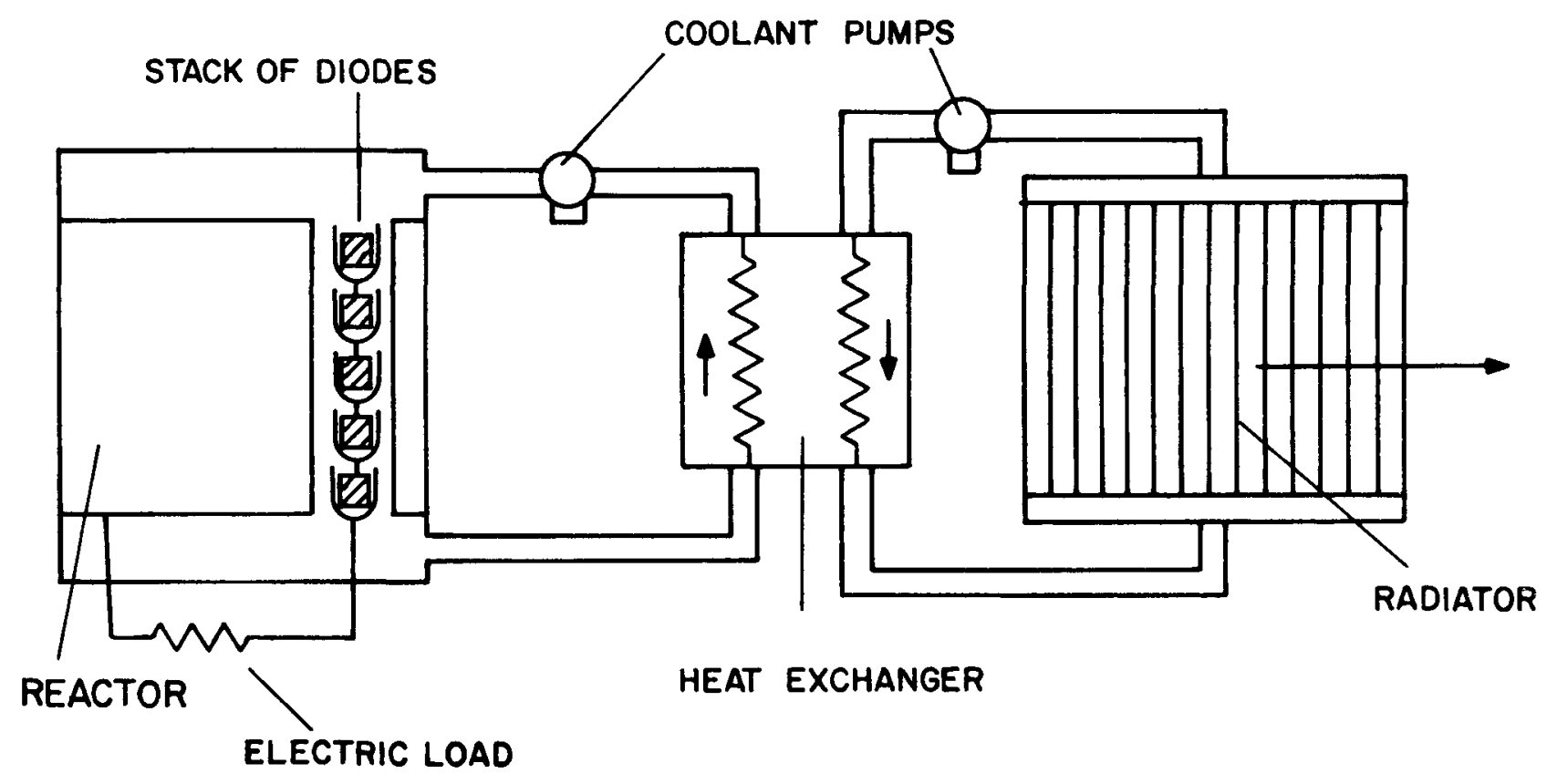

Figure 1.1. Overall Thermionic Reactor System. 
within a common sheath in a manner similar to the common flashlight. The resultant fuel element may consist of five to ten or more single diodes connected in series. A portion of the thermal power produced in the fuel is directly converted to electricity by the thermionic process and is drawn off through the electrical load. The remaining waste heat is conducted through the diode to the primary coolant, rejected across the heat exchanger to the secondary coolant, and subsequently radiated to space.

of prime interest in this research are those areas pertaining to the dynamic behavior of the system. Since the nuclear fuel is coupled intimately to the actual electrical output, the resulting response characteristics to electric load changes are quite dissimilar to those of conventional power plants. A1so, the physical makeup of this type of reactor leads to the possibility of destabilizing feedback contributions from the fuel region. This combination of effects makes it imperative that the dynamics of such a system be analyzed and understood.

Studies of thermionic reactor response have previously been aimed at investigations about a given full power operating point (Gronroos and Davis, 1968; Schock, 1968). While this allows the resulting analytical models to be simplified considerably, it also limits their areas of applicability. Consequently, the questions associated with large changes in the system variables, as for example during start-up, have to date been unanswered. 
With reference specifically to the start-up problem, the types of questions yet to be resolved include: How should the reactor be brought initially to power? When should the thermionic operation be phased in? Should the control system attempt to produce a constant emitter temperature or a constant output voltage? The purpose of this study is not necessarily to produce definitive answers to any of these questions nor to set the actual standards to be used but rather to provide a versatile model with which to conduct these kinds of studies.

\section{System Simulation Philosophy}

In the development of a system description for application to cases involving large changes in system state variables, an effort must be made to retain a reasonably accurate model over the entire anticipated ranges of these variables. This philosophy applies not only to the determination of the dynamic equations depicting the system's transient thermal response but also to the analysis required for calculation of the thermionic converter physics. These two areas constitute the entire system model and are each equally important.

Some sinulation detail has been sacrificed in order to keep the model, certainly a preliminary effort at best, within manageable bounds. For example, spatial considerations are minimized to an extent consistent with other assumptions and approximations within the system model. However, all regions of the system thought to be of importance in determining transient response are included so that their contributions may be accounted for at least approximately. This also permits the retention of realistic time constants for system simulation. 
The validity of some of the simplieations and ceduccions employed was investigated as a prelude to this work. The valinity of other assumptions, notably those assuciated with the use of average values of parameters and the converter physics, has been established by other researchers. These will be indicated in the course of developing the system mode1.

In order to allow general applications, the developed description is not restricted to any single specific reactor concept. The two aspects (i.e., the dynamic reactor system equations and the thermionic anaIysis), although related mathematically, are treated such that different systems may be analyzed.

Whenever actual systems are investigated during this study, realistic values for the describing parameters are employed so as to yield reasonable and constructive results. In addition, most of the effort is directed toward the open loop system response, since the study of control systems extends beyond the scope of this work.

\section{Content and Organization}

In the development of a useful system description, extensive effort must be devoted to each of the two previously mentioned main areas of concern. That is, before the model is actually utilized to study thermionic reactor start-up applications, much initial groundwork must be undertaken to develop the model and verify its worth.

Chater II illustrates how the actual describing equations for the reactor simulation are derived. This procedure is also applicable to a subsequent set of dynamic equations used in Chapter IV. Neutron 
kinetics and heat transfer are represented by "point" model descriptions, accomplished by eliminating spatial variations through volume-averaging techniques. The resulting equations are solved with a digital computer integration routine and initially tested by comparison with published results for a thermionic reactor concept simulated on an analog computer (Gronroos, 1967).

A procedure to calculate the various thermionic quantities of interest is discussed in Chapter III. The basis of this work is a digital computer program, SIMCON (Wilkins, 1968), for determining thermionic converter performance characteristics. Although SIMCON cannot be utilized directly in the dynamics model, it provides an excellent starting point from which to devise a usable scheme. This method by Wilkins is extended to allow applications to converters undergoing transients. The resulting method is then incorporated into the system model, and investigations are conducted to determine the relative worth of this more complicated approach over simpler thermionic descriptions.

Chapter IV constitutes an important segment of the research reported here. Response of the proposed analytical converter model is compared to direct transient experimental data obtained from a thermionic test facility. The accuracy and validity of the calculational scheme are demonstrated over wide ranges of actual diode operations.

An unanticipated and important consequence of the studies in Chapter IV is the demonstration of conditions leading to a 
potential inadequacy of spatial-averaging methods to describe the thermal response of converter systems. A method of accommodating this inadequacy in a relatively simple way is presented, and the validity established by comparison with experimental results.

Some results of simulated reactor system start-up are reported in Chapter $V$. Two sample cases of possible start-up schemes are illustrated. Both depict transitions from low initial thermal power to final equilibrium operating points. The first describes a procedure designed to maintain constant diode output voltage, while the second involves a constant emitter temperature start-up approach.

Chapter VI summarizes the conclusions and indicates recommendations for future work. Particular attention is paid to possible applications of this method to analyses involving multiple arrays of series-connected diodes. 
CHAPTER II

REACTOR SYSTEM ANALYSIS

The dynamic characteristics of a thermionic power plant are quite different from conventional reactor systems. Conventionally, load changes are reflected to the power region by variations in the coolant conditions, and are thus delayed effects. In the thermionic reactor, the fuel region is directly coupled to the electrical output through the thermionic process. Consequently, any perturbations in the electrical output are experienced almost instantly by the power producing region. In addition, compactness of the reactor for space applications requires that the fuel be highly enriched. This leads to the possibility of very smal1 positive prompt reactivity feedback from the fuel region. For these reasons, it is essential to investigate system response to various disturbances which may be introduced into such a thermionic reactor system.

A limited number of investigations along these lines are reported in the unclassified literature. These include Gronroos (1967), Gronroos and Davis (1968), Schock (1968), Weaver, Gronroos, Guppy and Davis (1969), and Brehm, Hetrick, and Schmidt (1969).

The earliest effort was that by Gronroos (1967), who used a complex analog computer simulation to investigate the open loop system response of such a system. Weaver, Gronroos, Guppy and Davis (1969) employed the same model to study controlled system dynamics. While the 
reactor description is reasonably comprehensive, the thermionic approximation used limited severely the region of application of the model.

The purpose of this work is to develop and establish the validity of a more general model capable of representing a thermionic reactor system over wide ranges of operation. As noted previously, the effort involved may be divided into two major areas; the reactor system representation and the converter processes description.

A suitable approach for representing the converter physics is presented in Chapter III. The next section of this chapter is devoted to the reactor model development. The method of solution of the corresponding set of equations is then discussed and, utilizing the same representation for converter physics as used by Gronroos (1967), sample cases to verify the results obtained by Gronroos are tested.

\section{Development of a System Model}

The two main reactor concepts possible arise from the location of the thermionic elements, either in-core or out-of-core. In this study, systems involving in-core converters are considered. Furthermore, for this type of reactor, the fuel region may be either internal or external to the emitter surface. In this study the internally-fueled concept is employed, thus heat is transferred outward to the coolant. However, a model similar to that developed here could also be applied to the externally-fueled concept.

While the converters are separated spatially, several may be connected electrically in series to form a "flashlight." Such a stack 
of diodes is shown in Figure 1.1. A more detailed representation of a series-connected diode is shown in Figure 2.1. Typical dimensions for the various converter regions are indicated.

Thermionic converters are inherently low-voltage, high-current devices, while the system electrical requirements call for high voltage and low current. Consequently, to enhance the electrical output, axial core sections consist of strings of several diodes connected in series instead of single long thermionic elements. In addition, long converters experience excessive lead losses and depressed thermionic operation resulting from axial temperature distributions. The analytical description of all diodes in such a reactor core incorporating explicitly the detailed power and coolant temperature distributions throughout the reactor is a complex formulation beyond the scope of this study. As a first attempt to produce a system model for analyzing transient thermionic reactor response, a gross spatial simplification is first made.

The Single Diode Thermionic Reactor Representation

The radial and axial power distributions of an operating reactor can be "flattened" to some extent by varying the fuel concentration. In fact, in order to utilize the diodes so that no single diode is severely limiting, axial power flattening must be carried out. Thus, an approximation which considers all diodes in the reactor to be operating under the same power and temperature conditions probably does not constitute a major restriction in terms of the dynamic representation. 


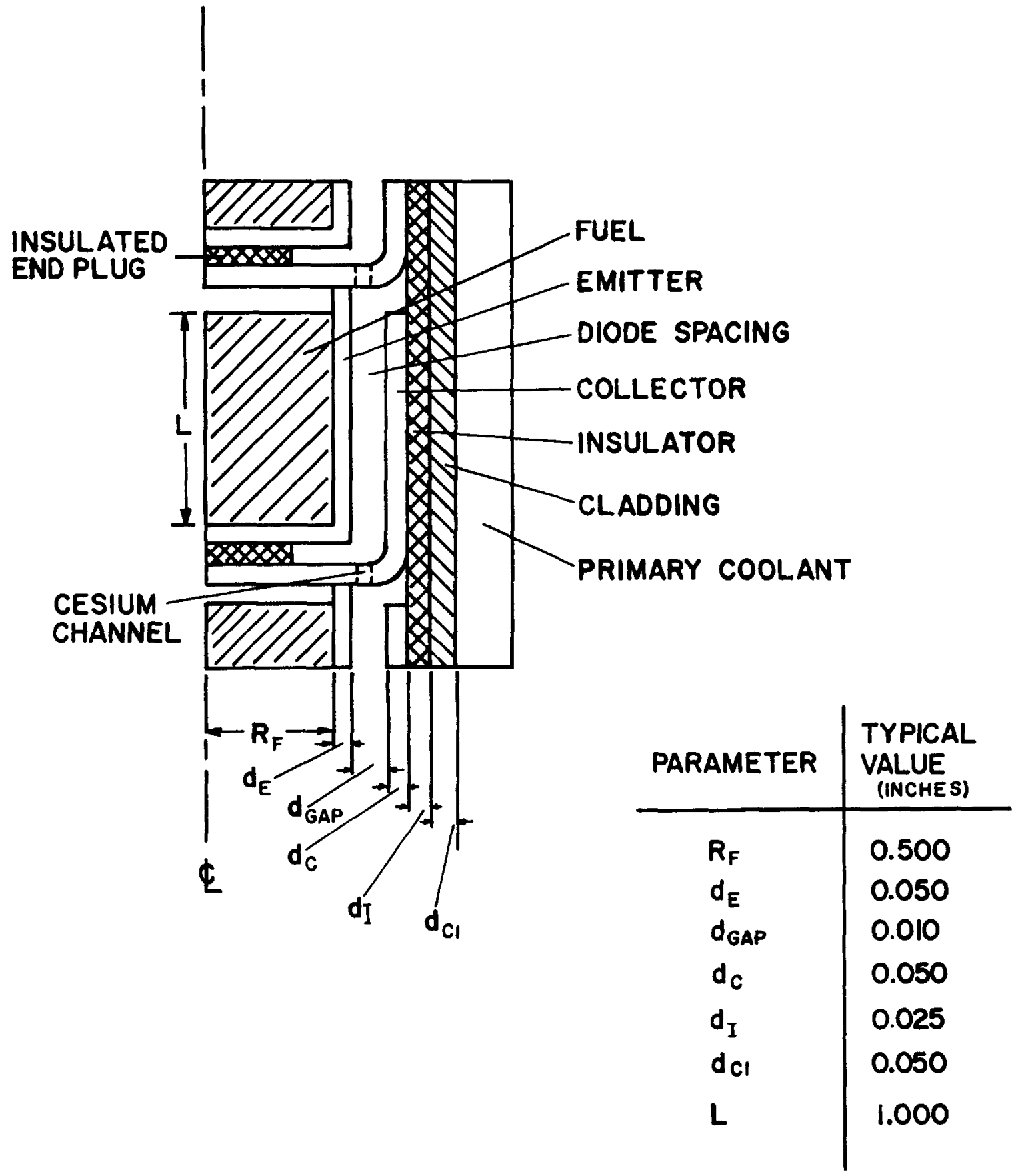

Figure 2.1. Typica1 Representation of Series-Connected In-core Diode Assembly for Flashlight Configuration 
By maintaining separate regions within the diode and treating each region individually, realistic dynamic behavior can be preserved, as will be demonstrated. The representation of the regions of interest for the single cylindrical converter model used is shown in Figure 2.2. The dynamic equations to be formulated require the specification of the time dependent reactor power as well as the temperatures for the various thermal regions. The reactor kinetics model is discussed first.

Reactor Kinetics Description. With the deletion of spatial dependence, the power density in the fuel is assumed identical at each point. Thus, the reactor kinetics can be suitably represented by the point reactor model. If the delayed neutron production is described by one average group, the reactor power equations may be written

$$
\begin{aligned}
& \frac{d n}{d t}=\frac{\rho(\rho-1)}{\ell} n+\lambda c^{\prime} \\
& \frac{d c^{\prime}}{d t}=\frac{\beta n}{\ell}-\lambda c^{\prime} \\
& \rho(t)=\rho_{0}+\sum_{j} \alpha_{j}\left(\bar{T}_{j}-\bar{T}_{j 0}\right)
\end{aligned}
$$

where

$$
\begin{aligned}
n(t) & =\text { neutron density or power } \\
c^{\prime}(t) & =\text { delayed neutron percursor concentration } \\
\rho(t) & =\text { reactivity of the system in fractions of } \beta \\
\beta & =\text { delayed neutron fraction } \\
\ell & =\text { neutron generation time }
\end{aligned}
$$




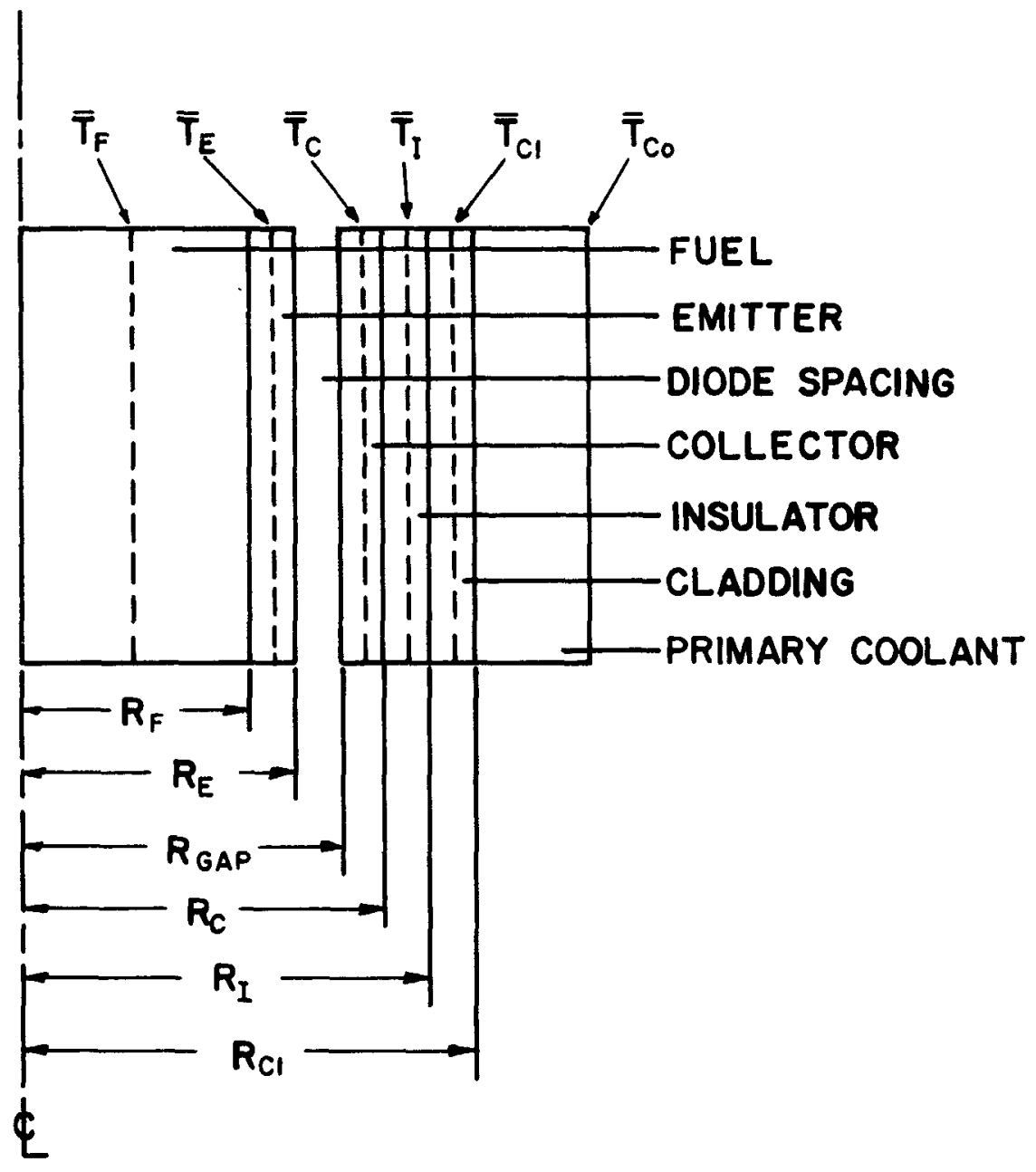

Figure 2.2. Representation of Regions for Single Diode Approximation 


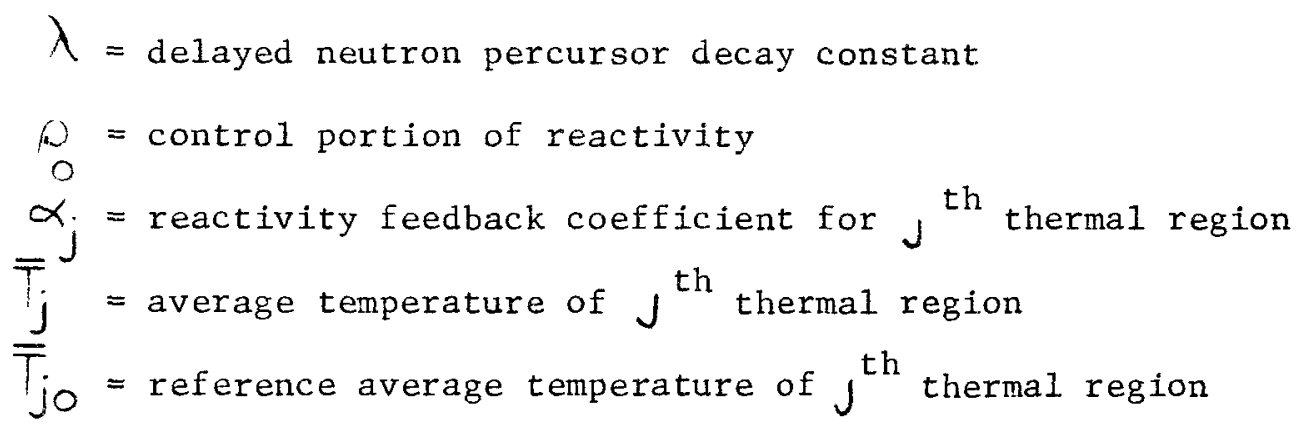

The parameters $\beta, \ell$, and $\lambda$ characterize the type of reactor and fueI under consideration. Since values of $\ell$ for fast spectrum thermionic reactors can be around $10^{-7}$ seconds, severe scaling problems can arise for any numerical analysis.

For this system, 1ittle accuracy is lost by applying the "prompt jump" approximation to Equation (2-1) (Hetrick, 1970). When $l$ is small and $\rho<\beta$, the term $\beta(\rho-1) n / l$ in $(2-1)$ is large and negative, while $\lambda c^{\prime}$ is large and positive. Thus, in such cases, $d n / d t$ is a very small difference between a large negative and a large positive number and may be neglected.

By applying this restriction and redefining $c^{\prime}$, the reactor equations (2-1) and (2-2) become

$$
\begin{aligned}
& n=\frac{\lambda c}{1-\rho} \\
& \frac{d c}{d t}=n-\lambda c
\end{aligned}
$$

where

$$
c \equiv \frac{\ell c^{\prime}}{\beta}
$$


The generation time $(\ell)$ has consequently been circumvented, and the scaling problems greatly alleviated. Instead of eliminating $c$ by differentiating $(2-4)$, the equations are left in this form for computer programming purposes. Thus, the complicated specification of $d p / d t$ is not required. It may be noted that the order of the systen: has been reduced by one.

Transient Therma1 Equations. The temperature regions of interest for the one diode model (Figures 1.1 and 2.2) are fuel, emitter, collector, insulator, cladding, reactor coolant, primary and secondary side of heat exchanger, and radiator. The thermal describing equations for each region may be written

$$
P_{j} V_{j} c_{p_{j}} \frac{d \overline{T_{j}}}{d t}=q_{\text {inj }}-7_{o u t_{j}}
$$

where

$$
\begin{aligned}
t & =\text { time } \\
F_{j} & =\text { density of } j \text { th region } \\
V_{j} & =\text { volume of } j^{\text {th }} \text { region } \\
q_{p_{j}} & =\text { specific heat of } j^{\text {th }} \text { region } \\
F_{j}(t) & =\text { spatially-averaged temperature of } j^{\text {th }} \text { region } \\
q_{i n j} & =\text { heat input rate to } j^{\text {th }} \text { region } \\
q_{\text {out }} & =\text { heat removal rate from } j^{\text {th }} \text { region }
\end{aligned}
$$

The product $P_{j} C_{P_{j}}$ represents the volumetric heat capacity of the $j^{\text {th }}$ region. 
The heat quantities $\left(q_{i n}, q_{\text {out }}\right.$ ) are generally described in terms of the spatially-averaged temperatures of adjacent regions and of the inherent physical parameters of the particular materials in question. Although these expressions are different from region to region, it is of interest to outline the general development of one of these "point" approximations to the dynamic heat transfer. In this instance, the fuel temperature transient equation is derived. Since the power is produced uniformly, the heat input rate to the fueled region is of the form

$$
q_{i n}=V_{F} \cdot r
$$

where $V_{F}$ is the fuel volume. The rate of heat conducted from the fuel into the emitter may be expressed

$$
\begin{aligned}
q_{\text {out }}=h_{F, E} A_{F}\left(\bar{T}_{F}-\bar{T}_{E}\right) & \\
A_{F} & =2 \pi R_{F} L_{F}
\end{aligned}
$$

where

$$
\begin{aligned}
& \text { subscript } F=\text { fuel, } E=\text { emitter } \\
& h_{F, E}=\text { effective heat transfer coefficient between fuel and } \\
& \text { emitter } \\
& A_{F}=\text { area of fuel-emitter interface } \\
& R_{F}=\text { outer fuel radius as shown in Figure } 2.2 \\
& L_{F}=1 \text { ength of fuel }
\end{aligned}
$$

To calculate $h_{F, E}$, use is made of the steady-state conduction law. Thus, Goutf in (2-9) may also be written: 


$$
q_{F} u_{F}=-\left.K_{F} A_{F} \frac{d T_{F}(r)}{d r}\right|_{r=R_{F}}
$$

where $K_{j}$ is the heat conductivity of the $j^{\text {th }}$ region. The spatial steady-state temperature variations for a cylindrical assembly in which the heat generation rate is uniform may be represented as

$$
\begin{aligned}
& T_{F}(r)=T_{F}(0)-\frac{n r^{2}}{4 h_{F}} \quad 0 \leq r \leq R_{F} \\
& T_{E}(r)=T_{E}\left(R_{E}\right)+\Gamma_{F}\left(R_{F}\right)-T_{E}\left(R_{\zeta}\right), \frac{\ln \left(R_{E} / r\right)}{\mid r\left(R_{E} / R_{F}\right)} \\
& \text { wnere } R_{F} \\
& T_{F}\left(R_{F}\right)=T_{E}\left(R_{F}\right)=T_{F}(c)-\frac{n R_{F}^{2}}{4 K_{F}}
\end{aligned}
$$

Equation (2-12) assumes no heat generation due to gamma radiation absorption in the emitter. The various $R$ are shown in Figure 2.2 . The analysis may proceed with the specification of the spatiallyaveraged temperatures.

A simplification is made here pertaining to all diode regions except the fuel. Since the fuel is assumed to be the sole powerproducing region, its steady-state temperature distribution is parabolic as shown by Equation (2-11). The spatial temperature variations for the emitter and all outer diode materials are logarithmic, as indicated by Equation (2-12). Due to the thinness of these regions compared to the fuel radius, these expressions do not deviate 
substantially from a linear function. Thus, while a normal cylindrical volume averaging technique is applied to the fuel

$$
\bar{T}_{F} \equiv \frac{1}{V_{F}} \int_{0}^{R_{F}} 2 \operatorname{rr}{ }_{F} T_{F}\left(r^{\prime}\right) d r=T_{F}(n)-\frac{n R_{F}^{2}}{8 K_{F}}
$$

negligible accuracy is lost by assuming the spatially-averaged temperatures for the remaining diode regions to be the linear average of the respective surface temperatures. Specifically, for the emitter this becomes

$$
\overline{T_{E}} \equiv \frac{1}{2}\left[T_{F}\left(R_{F}\right)+T_{E}\left(R_{E}\right)\right]
$$

This assumption greatly reduces the required mathematics for the averaging procedures and is quite adequate for the actual situation involved.

The heat transfer coefficient $\left(h_{F, E}\right)$ in (2-9) may be determined with the stipulation of the interface condition denoting continuity of heat across a boundary. This is of the form

$$
-\left.K_{F} A_{F} \frac{d T_{F}(r)}{d r}\right|_{r=R_{F}}=-\left.K_{E} \Delta_{F} \frac{d^{T}(r)}{d r}\right|_{r=R_{F}}
$$

The substitution of the derivative of (2-11) into (2-10) yields an expression for the left-hand side of (2-9). Equations (2-14) and (2-15) are used in the right-hand side of (2-9). This yields

$$
\frac{{ }_{n} R_{F}}{2}=h_{F E}\left\{T_{F}\left(0-\frac{{ }^{2} R_{F}^{2}}{3 K_{F}}-\frac{1}{2} \Gamma_{F}\left(R_{F}\right)+T_{E}\left(R_{E}\right)\right]\right\}
$$


Use of the derivatives of (2-11) and (2-12) in (2-16) and solution of the resulting equation for $T_{E}\left(Q_{E}\right)$ gives

$$
\left.T_{L} ! R_{E}\right)=T_{F}\left(R_{F}\right)-\frac{n R_{F}^{2}}{2 K_{E}}-\ln \left(R_{E} / R_{F}\right)
$$

Equation (2-13) may be solved for $T_{F}(0)$ in terms of $T_{F}\left(R_{F}\right)$. Substitution of this and (2-18) into (2-17) eliminates $n$ and the interface temperatures to produce

$$
h_{F, E}=\frac{2}{R_{F}}\left[\frac{1}{1 / 2 K_{F}+\ln \left(R_{E} / R_{F}\right) / K_{E}}\right]
$$

Thus, the representation of the average fuel temperature may be expressed by the equation

$$
P_{F} V_{F C_{F}} \frac{d \overline{T_{F}}}{d t}=V_{F} \cdot n-h_{F, E} \AA_{F}\left(\bar{T}_{F}-\bar{T}_{E}\right)
$$

In writing Equation (2-20), as well as any subsequent similar expressions, it is assumed for transient analysis that the spatial temperature distributions remain unchanged from the initial distributions. Previous studies have been conducted in this area pertaining to the fuel region in particular (Landrot, Bliaux, and List, 1965; Gronroos, 1967). For the case of uniform heat generation, it was found that the fuel temperature profile remained essentia1ly parabolic during transients. This earlier research also demonstrated that the representation of the fuel by a single lumped region produced almost identical 
results with the situation where up to six radial fuel regions were considered.

Thus, the forms of the distribution functions represented in (2-11) and (2-12) for the fuel and emitter respectively, are assumed to remain constant during transients. This further implies that heat transfer terms in the form of Equation (2-9) are valid, with the temperatures now time dependent.

The heat received by the emitter is that conducted in from the fuel region. The heat transferred from the emitter across the diode gap to the collector is determined by analysis of the thermionic processes involved. The detailed representation of these processes is discussed in Chapter III. A simple approach to this representation is shown later in this chapter. In general, the emitter thermal equation is of the form

$$
\begin{gathered}
P V_{E} V_{E} C_{E} \frac{d \overline{T_{E}}}{d t}=h_{F, E} A_{F}\left(\bar{T}_{F}-\bar{T}_{E}\right)-A_{E} a_{E}^{\prime \prime} \\
A_{E}=2 \pi R_{E} L_{E}
\end{gathered}
$$

where $q_{E}^{\prime \prime}$ is the emitter heat flux as set by the thermionic processes. The collector heat flux (denoted $q_{C}$ ") is simply described by the emitter heat flux minus the electrical power produced. While this is not strictly true, the other minor contributions and losses to the collector heat term are neglected in this analysis. Thus, the equation is written

$$
P_{C} V_{C} c_{P_{C}} \frac{d \bar{T}}{d t}=A_{E} q_{C}^{\prime \prime}-h_{C, I} A_{C}\left(\bar{T}_{C}-\overline{T_{I}}\right) \quad A_{C}=2 \pi P_{C} L_{C}
$$


where the effective heat transfer coefficient between the collector and insulator $\left(h_{C_{1} I}\right)$ is given by

$$
h_{C_{1} I}=\frac{2}{R_{C}}\left[\frac{1}{\ln \left(R_{C} / R_{G A P}\right) / K_{C}+\ln \left(R_{I} / R_{C}\right) / K_{I}}\right]
$$

The insulator equation may be expressed in a similar fashion

$$
P_{I} V_{I} C_{D_{I}} \frac{d T}{d t}=h_{C I} A_{C}\left(\bar{T}_{C}-\bar{T}_{I}\right)-h_{I C I} A_{I}\left(\bar{T}_{I}-\bar{T}_{C l}\right)
$$

where

$$
h_{I, C l}=\frac{2}{R_{I}}\left[\begin{array}{c}
1 \\
1,1 K_{I} / R_{C} / K_{I}+\ln \left(R_{C l} / R_{I}\right) / K_{C l}
\end{array}\right]
$$

While the heat received by the cladding is described by the last term of $(2-24)$, the determination of the heat conducted into the reactor coolant results in a different form for $h_{\mathrm{Cl}, \mathrm{CO}}$. Specifically

$$
\begin{aligned}
q_{\text {out }}=q_{\text {in }}=h_{C l, c o} A_{C l}\left(\bar{T}_{C l}-\bar{T}_{C o}\right) \\
=A_{C l}=2 \pi R_{C l} L_{C l}
\end{aligned}
$$

where $\bar{T}_{C O}$ is assumed to be the average bulk coolant temperature adjacent to the reference diode. The interface Equation (2-16) in this instance becomes

$$
\begin{aligned}
& -K_{C 1} A_{c 1} \frac{d T_{C 1}(r)}{d r} \mid=h A_{c 1}\left[T_{c 1}\left(R_{c 1}\right)-\bar{T}_{c o}\right] \\
& r=R_{C l}
\end{aligned}
$$


where $h$ is the actual heat transfer coefficient between the cladding surface temperature and the bulk coolant temperature. This quantity is determined from various flow parameters associated with the particular coolant and cladding material under investigation. The resulting expression for the effective heat transfer coefficient coupling the cladding to the coolant is

$$
h_{C I, C O}=\frac{2}{R_{C l}}\left[\frac{1}{\left(n\left(R_{C l} / R_{I}\right) / K_{C l}+2 /\left(h R_{C l}\right)\right.}\right]
$$

Thus, the cladding transient equation is written as

$$
P_{C l} V_{C l} c_{P} \frac{d T_{C l}}{d t}=h_{I, C l} \Delta_{I}\left(T_{I}-\bar{T}_{C l}\right)-h_{C l, C O} A_{C l}\left(\bar{T}_{C l}-\bar{T}_{C O}\right)
$$

If the single reference diode being considered is assumed to be the central converter in any given axial string of thermionic elements (see Fig. 2.3), the average bulk coolant temperature as used in (2-27) and (2-29) may be represented by

$$
\bar{T}_{c o} \equiv \frac{1}{2}\left(\bar{T}_{C O_{i}}+\bar{T}_{C o_{e}}\right)
$$

where

$$
\begin{aligned}
& T_{\mathrm{CO}} \quad=\text { average bulk coolant temperature at reactor inlet } \\
& T_{\mathrm{CO}_{e}} \quad=\text { average bulk coolant temperature at reactor outlet }
\end{aligned}
$$

as shown in Figure 2.3.

Since all diodes in the reactor are assumed to duplicate the operation of the reference device, the heat received by the coolant 


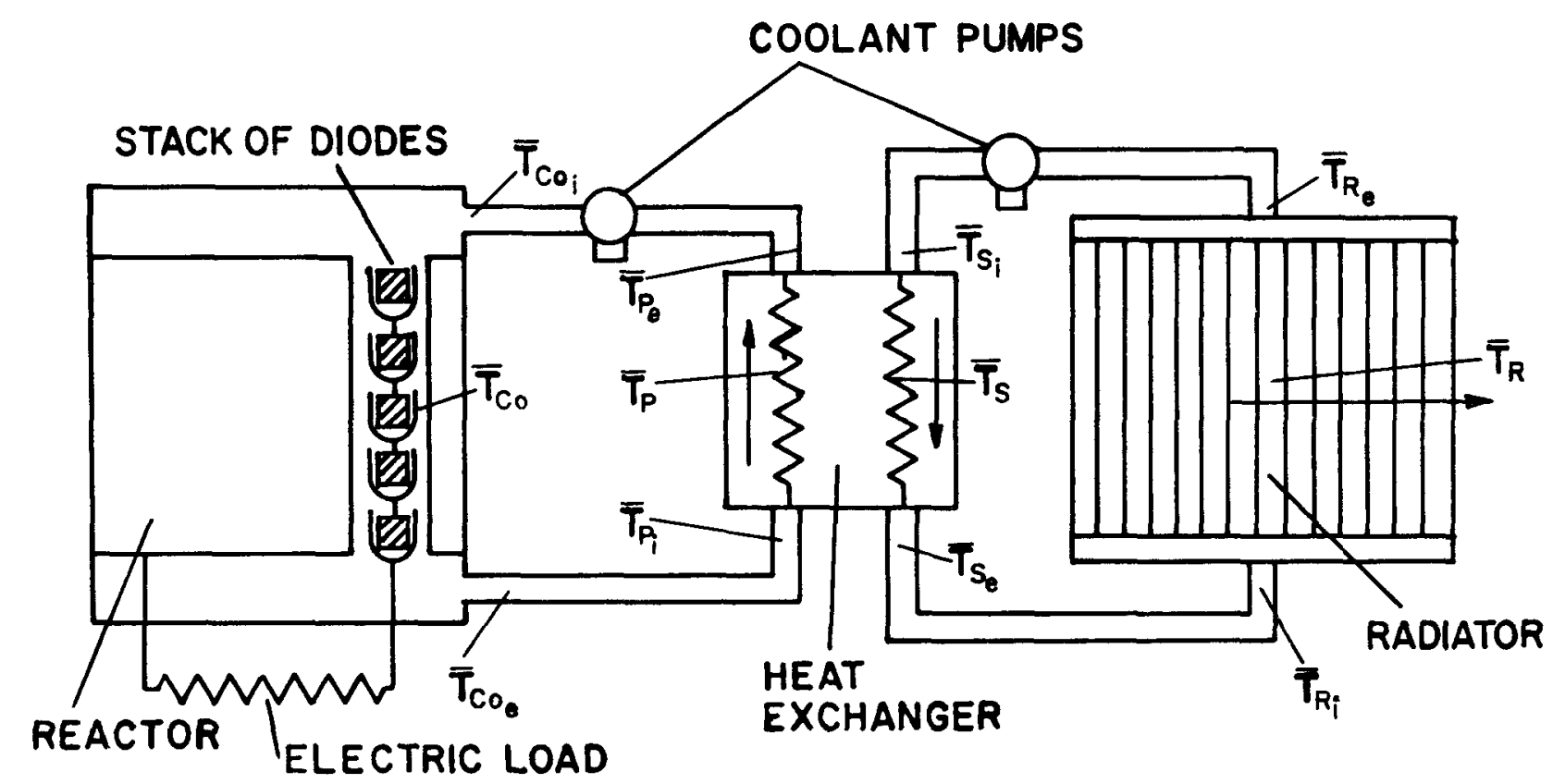

Figure 2.3. Reactor System Diagram Showing Location of Coolant Loop Temperatures 
from any string is given by the last term of (2-29) multiplied by the number of elements in the stack. The heat removed from the reactor by the coolant may be expressed in terms of the inlet and outlet temperatures and certain coolant parameters. Thus, the coolant equation for any string of diodes is

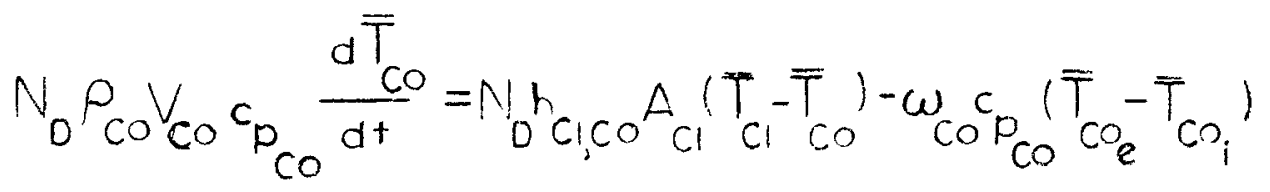

where

$$
\begin{aligned}
V_{C O} & =\text { coolant volume per diode } \\
N_{O} & =\text { number of diodes per stack } \\
\omega_{C O} & =\text { coolant mass flow rate } \\
A_{C l} & =2 \pi R_{C l} L_{C l}=\text { cladding surface area }
\end{aligned}
$$

By a similar analysis, the total heat reaching the primary side of the heat exchanger is the last term of (2-31) multiplied by the number of diode stacks in the reactor core. The resulting primary side thermal equation becomes

$$
\begin{aligned}
& M_{P} c_{p} \frac{d T_{P}}{d t}=N_{P} \omega_{C O} c_{P_{C O}}\left(\bar{T}_{P_{i}}-\bar{T}_{P_{e}}\right)-\left({ }_{H} A_{H}\left(\overline{T_{P}}-\overline{T_{S}}\right)\right. \\
& \bar{T}_{P} \equiv \frac{1}{2}\left(\bar{T}_{P_{e}}+\bar{T}_{P_{i}}\right)
\end{aligned}
$$

where

$$
\begin{aligned}
& M_{P}=P_{P} V_{P}=\text { mass of primary side of heat exchanger } \\
& \Gamma_{P}=\text { number of diode stacks in parallel } \\
& \bar{T}_{P_{i}}=\text { average bulk coolant temperature at primary side inlet }
\end{aligned}
$$




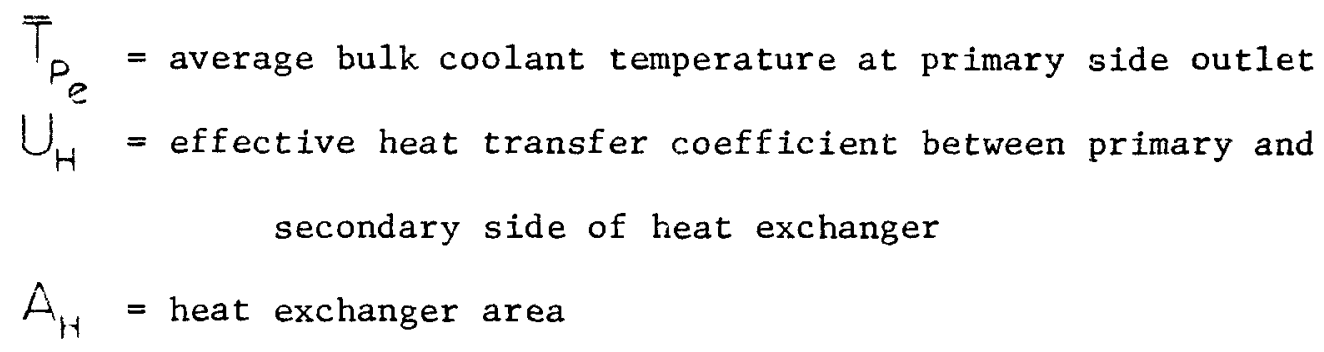

In general, the coolant is delayed because a finite time is required for the coolant to leave the reactor and enter the primary side. In addition, the piping system has a finite heat capacity and heat loss rate. These factors all contribute to an effective delay in the transfer of thermal energy from the reactor to the heat exchanger and similarly back to the reactor. To account for these effects, equations may be modeled by the form

$$
\begin{aligned}
& \bar{T}_{f_{i}}(t)=\bar{T}_{C_{e}}\left(t-T_{1}\right) \\
& \bar{T}_{C o}(t)=\bar{T}_{P_{e}}\left(t-T_{2}\right)
\end{aligned}
$$

where $\mathcal{T}_{j}$ is the $j{ }^{\text {th }}$ time delay and the actual energy loss is neglected.

The same treatment of coolant delay is valid for the secondary side and radiator analysis. The describing equation for this portion of the heat exchanger is

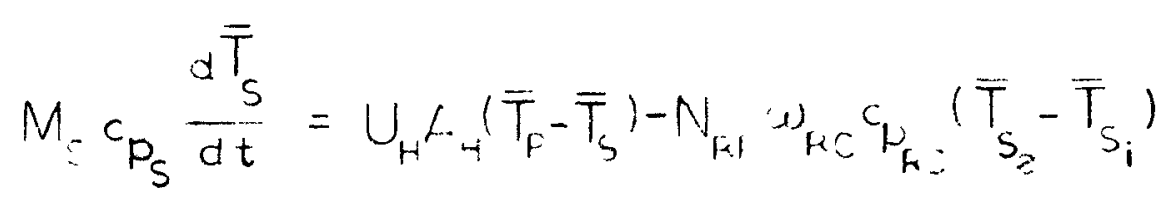




$$
\bar{T}_{s} \equiv \frac{1}{2}\left(\bar{T}_{S_{i}}+\bar{T}_{S_{e}}\right)
$$

where

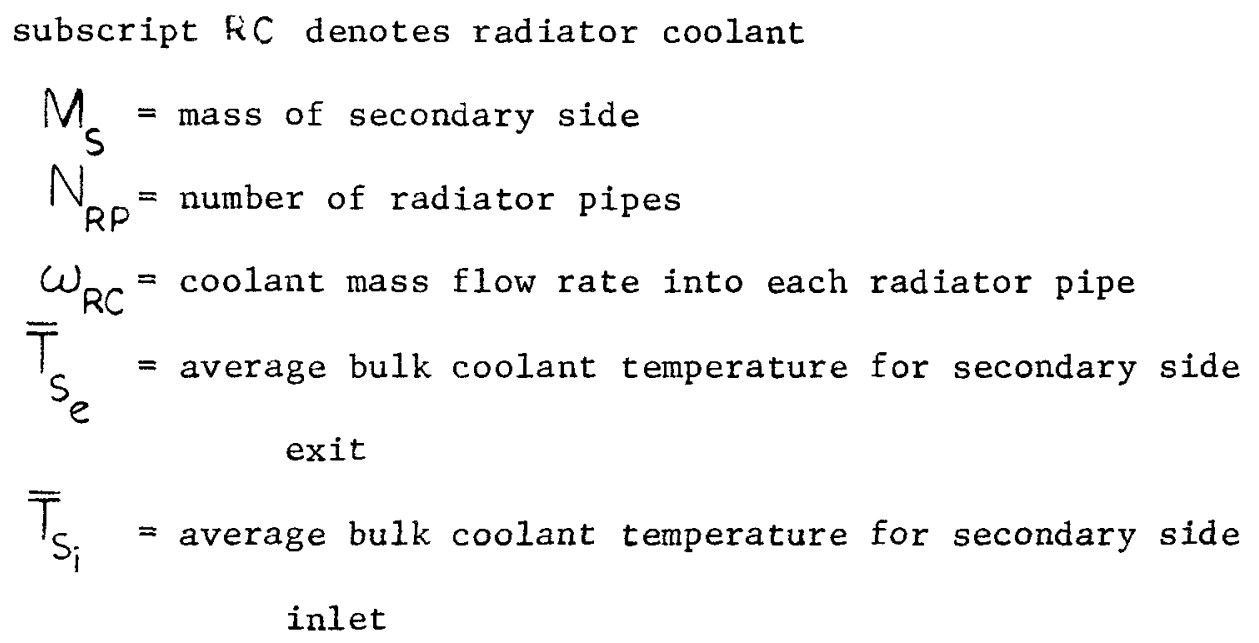

The radiator transient thermal equation for each pipe (assumed identica1)_may be expressed

$P_{R} V_{R} c_{P_{R}} \frac{d T_{R}}{d t}=\omega_{R C} c_{P_{R C}}\left(\bar{T}_{R}-\bar{T}_{R}\right)-\sigma \epsilon_{R} A_{R} \bar{T}_{R}^{4}$

$$
\bar{T}_{R} \equiv \frac{1}{2}\left(\bar{T}_{R_{e}}+\bar{T}_{R_{i}}\right)
$$

where

$$
\begin{aligned}
T_{R_{i}} & =\text { average bulk coolant temperature at radiator inlet } \\
T_{R_{e}} & =\text { average bulk coolant temperature at radiator exit } \\
\epsilon_{R} & =\text { emissivity of radiator surface } \\
\sigma & =\text { Stefan-Boltzmann constant }
\end{aligned}
$$




$$
\Delta_{R}=\text { surface area of each radiator pipe }
$$

The secondary coolant delay times may be included as

$$
\begin{aligned}
& \bar{T}_{S_{1}}(t)=\bar{T}_{R_{e}}\left(t-T_{3}\right) \\
& \bar{T}_{R_{i}}(t)=\bar{T}_{S_{e}}\left(t-T_{4}\right)
\end{aligned}
$$

The last term in (2-38) assumes that no appreciable temperature drop occurs by heat conduction through the radiator walls and that the fourth power of the ambient environmental temperature is negligible when compared to $\bar{T}_{R}^{4}$.

This completes the developnent of a general set of equations to describe the transient heat transfer. Thus Equations (2-3) through (2-41) constitute a reasonably complete model for the representation of a diode thermionic reactor system in which coolant transport delays are included.

Although this coolant $\mathrm{Iag}$ is snown to be easily accounted for, the present analysis can be simplified if these effects are neglected. The general response of the overall system is not thought to be greatly altered by this deletion for realistic situations. Therefore, in all transient cases reported in this study, the delay times have been assumed to be zero, although the actual validity of this assumption remains to be established.

For this instance of no coolant transport lag, the resulting system equations may be reduced by two, with no loss of accuracy. When 
all exit coolant temperatures are equal to the corresponding inlet temperatures of their respective adjacent regions, it follows that

$$
\begin{aligned}
& \bar{T}_{C o}(t)=\bar{T}_{P}(t) \\
& \bar{T}_{S}(t)=\bar{T}_{R}(t)
\end{aligned}
$$

Thus, the reactor coolant and primary side equations ((2-31)

and (2-32)) may be collapsed to give

$$
\begin{aligned}
\left(N_{S} \mu_{P}, P_{C:} C_{P}+M_{P O} c_{P}\right) \frac{d T_{C O}}{d t} & =N_{S} N_{P} h_{C I, C O} A_{C l}\left(\bar{T}_{C l}-\bar{T}_{C O}\right)- \\
& -\left(U_{H} A_{H}\left(\bar{T}_{C O}-\bar{T}_{R}\right)\right.
\end{aligned}
$$

Also, addition of (2-36) with (2-38) produces

$\left(M_{S} c_{P_{S}}+N_{R I} P_{R} V_{R} c_{P_{R}}\right) \frac{d \bar{T}_{R}}{d t}=U_{H} A_{H}\left(\bar{T}_{C O}-\bar{T}_{R}\right)-N_{R P} \sigma \epsilon_{R} A_{R} \bar{T}_{R}^{4}$

Thus, four equations are reduced to two. For convenience, the final resulting set of equations for the reactor system model to be studied is listed in Table 2.1 and the respective reference equation numbers are noted.

No restriction has been placed on any of the physical parameters involved during the development of the system transient equations. Thus, the inclusion of their temperature dependence is possible. In the cases reported here, however, constant values for the material properties under consideration are assumed. 
TABLE 2.1 .

Reactor System Equations

Power:

$$
\begin{aligned}
& n={ }_{1-\rho}^{\lambda c}(2-4) \quad f=P_{c}+j_{j}^{j} \alpha_{j}\left(T_{j}-\bar{T}_{j o}\right) \\
& \frac{d c}{d t}=n-\lambda c
\end{aligned}
$$

Fuel:

$$
\stackrel{U V}{V} c_{p_{F}} \frac{d T_{F}}{d t}=V_{F} n-h_{F, E} L_{r}\left(T_{r}-\bar{T}_{E}\right)
$$

Emitter:

$$
P_{E} V_{E} C_{E} \frac{d \overline{i_{E}}}{d t}=h_{F, E} A_{F}\left(\bar{T}_{F}-\bar{T}_{E}\right)-A_{E} q_{E}^{\prime \prime}
$$

Collector:

\section{Insulator:}

$$
{ }_{C} V_{C} c_{P_{C}} \frac{d \bar{c}}{d t}=A_{E} c_{C}^{\prime \prime}-h_{C, I} A_{C}\left(\overline{T_{C}}-\bar{T}_{I}\right)
$$

$$
P_{I}^{V_{I} c_{P_{I}}} \frac{d_{I}^{\bar{I}}}{d t}=h_{C, I} A_{C}\left(\bar{T}_{C}-\bar{T}_{I}\right)-h_{I, C l} A_{I}\left(\bar{T}_{I}-\bar{T}_{C l}\right)
$$

Cladding :

$$
P_{C l} V_{C l} c_{P_{C 1}} \frac{d \bar{T}_{C 1}}{d t}=h_{I, C 1} L_{I}\left(\bar{T}_{I}-\bar{T}_{C l}\right)-h_{C 1, C O} \Delta_{C l}\left(\bar{T}_{C l}-\bar{T}_{C O}\right)
$$

Coolant :

$$
\begin{aligned}
\left(N_{S} P_{P} P_{C O} V_{C O} c_{P_{C O}}+M_{P} c_{P}\right) \frac{d \bar{T}}{d t} & =M_{S} N_{P}{ }^{h} C l, C O A_{C l}\left(\bar{T}_{C l}-\bar{T}_{C O}\right)- \\
& -U_{H} A_{H}\left(\bar{T}_{C O}-\bar{T}_{R}\right)
\end{aligned}
$$

Radiator:

$$
\left(M_{S} c_{p_{S}}+N_{R H} P_{R} V_{R} c_{P}\right) \frac{d \bar{T}_{R}}{d t}=\bigcup_{H} A_{H}\left(\bar{T}_{C O}-\bar{T}_{R}\right)-N_{R P} \sigma \epsilon_{R} A_{R} \bar{T}_{R}^{4} \quad(2-45)
$$


With the specification of a set of dynamic equations, the problem of the actual solution of these equations must be addressed. This aspect is discussed in the next section.

Solution Methodology

The resulting system equations, as shown in Table 2.1 , contain various nonlinearities. In particular, the determination of ${ }^{\prime \prime}{ }_{E}$ (discussed in detail in Chapter III) requires a complex iterative analysis. For these reasons, the use of a digital instead of analog computer is selected. In this study, the $\operatorname{CDC} 6400$ digital computer at the University of Arizona Computer Center is employed to perform the numerical computations.

The solution of the set of differential equations describing the system transient response necessitates use of an integration scheme. Preliminary work in this study was done with a routine obtained from Los Alamos (Lewis and Stoval1, 1965). However, a more advanced and readily usable method was later secured from the Jet Propulsion Laboratory (Krogh, 1969).

This scheme consists of a very fast predictor-corrector method and easily handles a set of nonlinear first-order differential equations. All transient results shown here are run with this newer integration routine. A listing of the program is included in the appendix.

\section{Comparison Between Analog and Digital Approaches}

In order to check the validity of the reactor model and integration method, comparison studies were conducted. The model used 
for these tests was that reported by Gronroos (1967). His treatment involved a large and complex analog computer simulation, mentioned previously.

Gronroos' system of equations described a thermionic reactor plant comparable to that shown in Table 2.1. In making these comparison runs, the equations and coefficients used in that study were reproduced exactly. The materials and associated physical parameters are listed in Table 2.2. The actual differential equations and respective time constants are shown in Table 2.3 .

A Simplified Model of Converter Energy Transport

The thermionic analysis is handled in both models by assuming that a Richardson-Dushman (Angrist, 1965) approach is valid. In order to specify $q_{E}^{\prime \prime}$ (the emitter heat flux), the diode current must be calculated. This is approximated by writing Richardson-Dushman electron emission current densities for the emitter and collector surfaces. Specifically

$$
\begin{gathered}
J=J_{E}-J_{C} \\
\left.J_{E}=f_{E} A T_{E}^{2} \operatorname{exi-} \frac{\left.\Gamma_{C}+J R_{L}\right)}{K T_{E}}\right] \\
J_{C}=f_{C} A T_{C}^{2} \exp \left(-e \phi_{C} / K T_{C}\right) \\
V_{O}=J \cdot R_{L}
\end{gathered}
$$


TABLE 2.2

Equilibrium Conditions and System Parameters for Reactor Model Comparisons

\begin{tabular}{|c|c|c|c|c|c|c|c|c|}
\hline \multicolumn{9}{|c|}{ System Material Parameters } \\
\hline Item & Unit & $\begin{array}{l}\text { Fue1 } \\
\text { UC }\end{array}$ & $\begin{array}{l}\text { Emitter } \\
\quad W\end{array}$ & $\begin{array}{c}\text { Collector } \\
\mathrm{Nb}\end{array}$ & $\begin{array}{c}\text { Insulator } \\
\mathrm{Al}_{2} \mathrm{O}_{3}\end{array}$ & $\begin{array}{l}\text { Cladding } \\
\mathrm{Nb}\end{array}$ & $\begin{array}{c}\text { Coolant } \\
\mathrm{Li}-7\end{array}$ & $\begin{array}{c}\text { Radiator } \\
\text { SS }\end{array}$ \\
\hline$\overline{\mathrm{T}}_{i}$ & ${ }^{\circ} \mathrm{K}$ & 2150 & 2050 & 1300 & 1266 & 1232 & 1225 & 1185 \\
\hline$\rho_{j}$ & $\mathrm{gm} / \mathrm{cm}^{3}$ & 9.9 & 18.00 & 8.30 & 3.2 & 8.35 & 0.44 & 8.35 \\
\hline $\mathrm{C}_{\mathrm{P}}$. & $\mathrm{w}-\mathrm{s} / \mathrm{gm}-{ }^{\circ} \mathrm{K}$ & 0.268 & 0.188 & 0.31 & 1.19 & 0.31 & 4.14 & 0.837 \\
\hline$k_{j}^{j}$ & $\mathrm{w} / \mathrm{cm}-{ }^{\circ} \mathrm{K}$ & 0.230 & 1.59 & 0.63 & 0.0347 & 0.63 & & \\
\hline$R_{j}$ & $\mathrm{~cm}$ & 1.500 & 1.60 & 1.725 & 1.755 & 1.85 & & 1.13 \\
\hline$L_{j}$ & $\mathrm{~cm}$ & 5.00 & 5.00 & 5.00 & 6.0 & & & 200 \\
\hline$\alpha_{j}$ & $\$ /{ }^{\circ} \mathrm{K}$ & $+4 \times 10^{-5}$ & $-8 \times 10^{-5}$ & $-1.5 \times 10^{-4}$ & 0 & $-1.5 \times 10^{-4}$ & $-1.5 \times 10^{-4}$ & 0 \\
\hline
\end{tabular}

\begin{tabular}{|c|c|c|c|c|c|c|c|c|c|c|c|}
\hline \multicolumn{6}{|c|}{ Thermionic Parameters } & \multicolumn{6}{|c|}{ Other Parameters } \\
\hline Item & Unit & Value & Item & Unit & Value & Item & Unit & Value & Item & Unit & Value \\
\hline $\mathrm{J}$ & $\mathrm{amp} / \mathrm{cm}^{2}$ & 10.0 & $\mathrm{~d}$ & mil & 10.0 & $\mathrm{n}_{0}$ & watt $/ \mathrm{cm}^{3}$ & 82.0 & $\mathrm{~N}_{\mathrm{RP}}$ & none & 50 \\
\hline$J_{E}$ & $a m p / c^{2}$ & 11.0 & $\mathrm{~N}_{\mathrm{S}}$ & none & 5 & $\mathrm{~h}$ & $\mathrm{w} / \mathrm{cm}^{2}-{ }^{\circ} \mathrm{K}$ & 6.0 & $\varepsilon_{\mathrm{R}}$ & none & 0.8 \\
\hline $\mathrm{J}_{\mathrm{C}}$ & $\operatorname{amp} / \mathrm{cm}^{2}$ & 1.0 & $\mathrm{~N}_{\mathrm{P}}$ & none & 50 & $\mathrm{M}_{\mathrm{P}}$ & $\mathrm{Kgm}$ & 29.5 & $\lambda$ & $\sec ^{-1}$ & 0.1 \\
\hline$q_{E}^{\prime \prime}$ & watt $/ \mathrm{cm}^{2}$ & 58.3 & $\varepsilon_{E}$ & none & 0.3 & $\mathrm{C}_{\mathrm{P}_{\mathrm{D}}}$ & $\mathrm{w}-\mathrm{s} / \mathrm{gm}-{ }^{\circ} \mathrm{K}$ & 0.837 & $\beta$ & none & 0.0064 \\
\hline $\mathrm{v}_{0}$ & volt & 0.6 & $\varepsilon_{\mathrm{C}}$ & none & 0.3 & ${ }_{\mathrm{M}}^{1} \mathrm{P}$ & $\mathrm{Kgm}$ & 29.5 & & & \\
\hline$R_{L}$ & $\Omega-\mathrm{cm}^{2}$ & 0.06 & $h_{E, C}$ & $\mathrm{w} / \mathrm{cm}^{2}-{ }^{\circ} \mathrm{K}$ & 0.0165 & $\mathrm{C}_{\mathrm{P}}$ & $\mathrm{w}-\mathrm{s} / \mathrm{gm}-{ }^{\circ} \mathrm{K}$ & 0.837 & & & \\
\hline$\phi_{\mathrm{C}}$ & ev & 2.1 & & & & $\mathrm{U}_{\mathrm{H}} \mathrm{A}_{\mathrm{H}}^{\mathrm{S}}$ & watt $/{ }^{\circ} \mathrm{K}$ & 16200 & & & \\
\hline
\end{tabular}


33

TABLE 2.3 .

Dynamic Equations for Reactor System Comparisons

$$
\begin{aligned}
& n=\frac{0.1 c}{1-\rho} ; P=P_{j}+\sum_{j} \alpha_{j}\left(\bar{T}_{j}-\bar{T}_{j}\right) \\
& \frac{d c}{d t}=n-0.1 c \\
& \frac{d \bar{T}_{E}}{d t}=3.03: n-0.308 \geq\left(\overline{T_{F}}-\bar{T}_{E}\right) \\
& \frac{d T_{E}}{d t}=1.254\left(\overline{i r}_{F}-\bar{T}_{E}\right)-3.051 q_{E}^{\prime \prime} \\
& \frac{d T_{C}}{d t}=3.112 q_{C}^{\prime \prime}-5.635\left(T_{c}-T_{I}\right) \\
& \frac{d \bar{T}_{I}}{d t}=4.639\left(\bar{T}_{C}-\bar{T}_{I}\right)-4.539\left(\bar{T}_{I}-\bar{T}_{C l}\right) \\
& \frac{d \bar{T}_{C 1}}{d t}=6.928\left(T_{I}-T_{C l}\right)-3.154\left(T_{C l}-\bar{T}_{C O}\right) \\
& \frac{d T_{C O}}{d t}=3.095\left(\bar{T}_{C l}-\bar{T}_{C o}\right)-0.578\left(\bar{T}_{C O}-\bar{T}_{R}\right) \\
& \frac{d \bar{T}_{R}}{d t}=0.28\left(\bar{T}_{C O}-\bar{T}_{R}\right)-5.73 \times 10^{-12} \bar{T}_{R}^{4}
\end{aligned}
$$


where

$$
\begin{aligned}
& \text { subscript } E=\text { emitter; } C=\text { collector } \\
& 1=\text { net diode electron current } \\
& U_{j}=\text { electron emission current density from } j^{\text {th }} \text { surface } \\
& \bar{T}_{j}=\text { average temperature of } j^{\text {th }} \text { surface } \\
& V_{0}=\text { diode output voltage } \\
& R_{L}=10 a d \text { resistance } \\
& \phi_{C}=\text { collector work function } \\
& \AA=\text { Richardson constant } \\
& =\quad \text { electron charge } \\
& K=\text { Boltzmann constant } \\
& f \text { = patchiness factor for } j^{\text {th }} \text { surface } \\
& \text { With the determination of } J, q_{E}^{\prime \prime} \text { is calculated from: } \\
& q_{E}^{\prime \prime}=Q_{e}+Q_{r}+Q_{C S}
\end{aligned}
$$

where

$$
\begin{aligned}
& Q_{e}=\text { electron cooling } \\
& Q_{r}=\text { radiation cooling } \\
& \gamma_{C S}=\text { cesium conduction }
\end{aligned}
$$

These various terms are expressed

$$
\begin{aligned}
& Q_{e}=J\left(J \cdot R_{L}+\phi_{C}\right)+\frac{2 K}{e}\left(J_{E} \cdot \ddot{T}_{E}-U_{C} \cdot \bar{T}_{C}\right) \\
& \lambda_{r}=\frac{\sigma}{\left(1 / \epsilon_{E}+1 / \epsilon_{C}-1\right)}\left(\bar{T}_{E}^{4}-\bar{T}_{C}^{4}\right)
\end{aligned}
$$




$$
Q_{c s}=h_{E, c}\left(\bar{T}_{E}-\bar{T}_{i}\right)
$$

where

$$
\begin{aligned}
& \sigma=\text { Stefan-Boltzmann constant } \\
& \epsilon_{j}=\text { emissivity of } j \text { th surface } \\
& n_{E, C}=\text { effective heat transfer coefficient for cesium } \\
& \text { conduction }
\end{aligned}
$$

Equation (2-51) contains quantities to account for the electron kinetic energy components present in the postulated "uphil1" mode diode. The voltage profile across the converter for this mode appears as shown in Figure 2.4. Specification of the emitter work function is not required for this type approximation.

So that function generators for the exponential terms will not be required for the analog computer simulation, Equations (2-47) and (2-48) were differentiated and solved explicitly for $d J_{E} / d t$ and $d J_{C} / d t$. Consequently, the need to specify the patchiness factors ( ${ }_{E}$ and ${ }^{f_{C}}$ ) is eliminated. This same technique is used in the digital comparison.

Two transient cases are studied, with the digital results being directly compared to the analog computer response. They depict a step insertion of reactivity, and a step change in load resistance. 


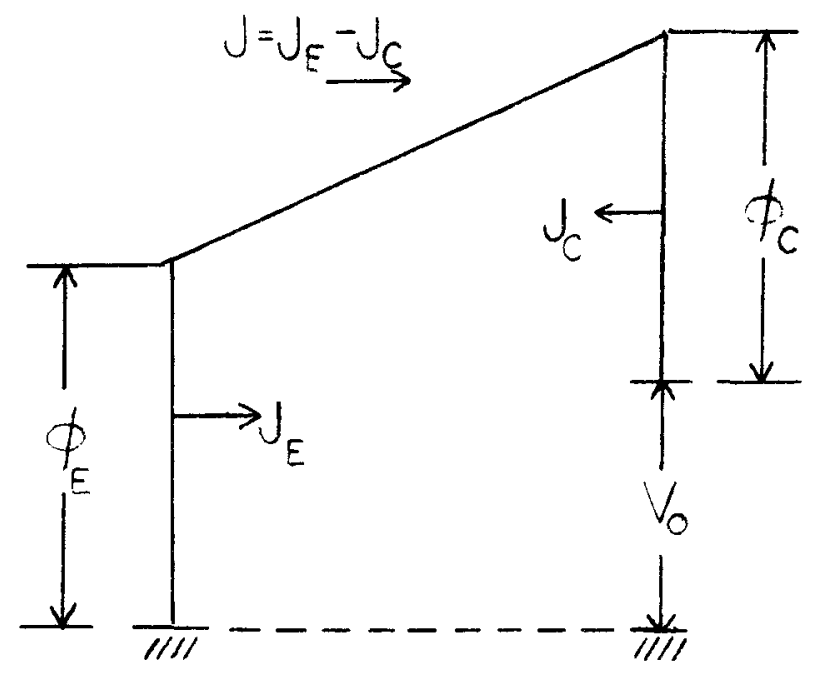

$J=$ net diode electron current

$J_{E}=$ emitter surface electron current density

$ل_{C}=$ collector surface electron current density

$\phi_{E}=$ emitter work function

$\phi_{C}=$ collector work function

$V_{0}=$ diode output voltage

Figure 2.4. "Uphill" Mode Voltage Profile for R-D Analysis 
Step in Power

With the reactor system operating at equilibrium power, a two-cent step in reactivity is inserted. This means that $\vec{r}$ in Equation (2-3) is incremented to $0.02 \beta$. A perturbation in reactivity such as this is considered small and is well within the region of validity of the prompt jump approximation.

The responses of several important variables are shown in Figure 2.5. The power density immediately increases and the system undergoes a transient while establishing a new equilibrium operating point. The power gradually stabilizes as the feedback contributions from the various thermal regions compensate for the input reactivity. As seen from these time traces, such an open loop thermionic reactor is very slow to respond due to the weakness of the stabilizing feedback effects inherent in the system. Thus, small reactivity perturbations lead to large changes in operating conditions.

The comparisons between analog and digital responses are close, but by no means exact. Since a discrepancy exists, a further test is conducted to determine which of the two solution methods is correct and where the source of the discrepancy lies.

Perhaps the most obvious next step is to ascertain if both models are accurately accommodating the nonlinear effects. These can sometimes introduce inaccuracies, particularly on analog computers. A $2 c$ step in reactivity does not constitute a large perturbation as can be seen from the results of Figure 2.5 (maximum changes in any of the variables are of order $15 \%$ ). It is therefore reasonable to 

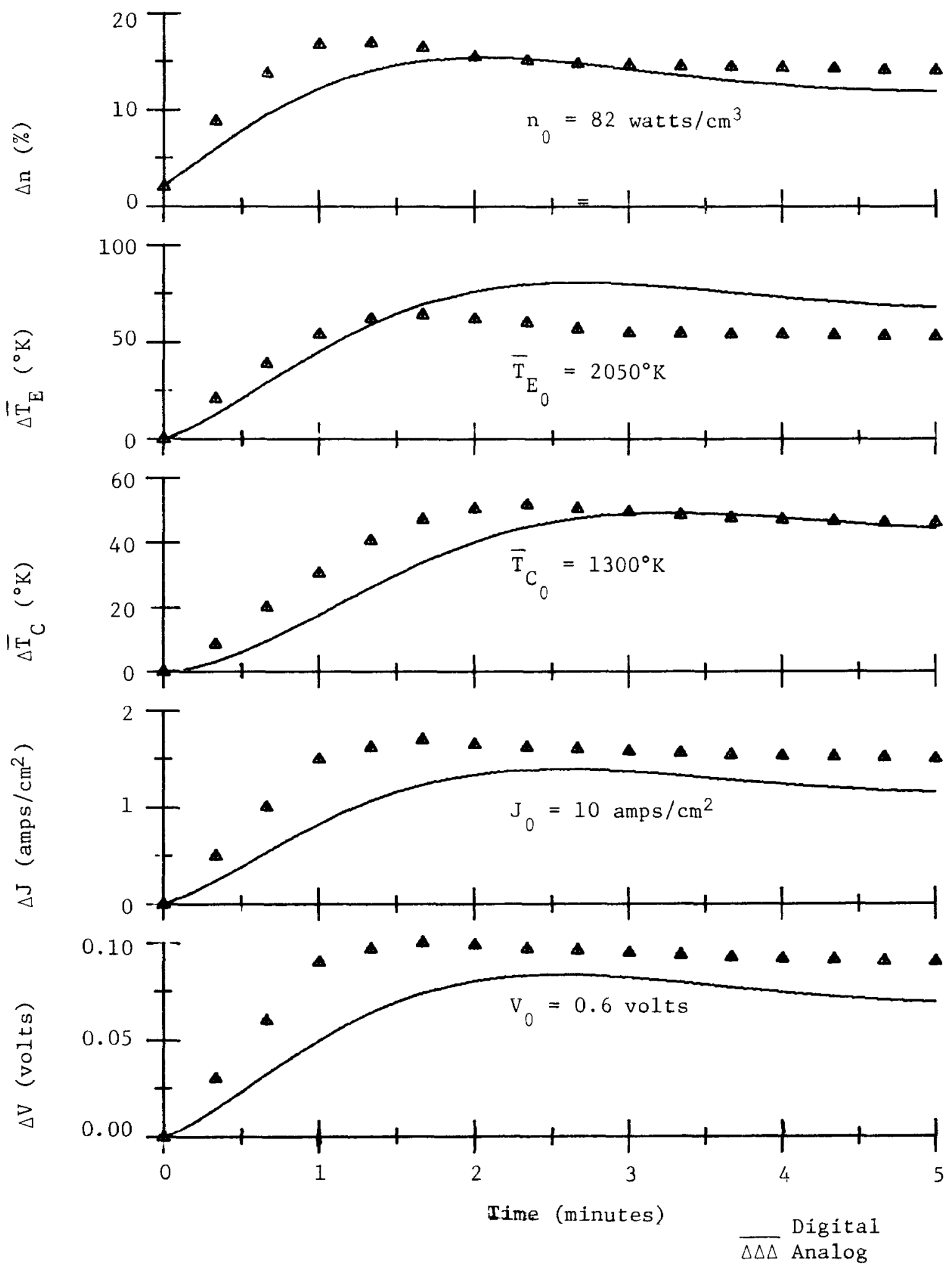

Figure 2.5. Comparison of Nonlinear System Response for $+2 c$ Step. 
postulate that a linearized approximation of the same model should result in almost the same response.

While the system equations for the digital computer model can be linearized and the corresponding response obtained, results from a corresponding linearization of the complete analog simulation are not available. However, responses from a reduced version of the general analog model are available and can be utilized for comparative purposes.

The equations for this second analog system resulted from the linearization and reduction of the general analog description to a set of four first-order differential equations involving composite regions. The four equations include the reactor power, a combined fuel-emitter region, the collector region, and a lumped heat rejection representation incorporating all regions from the insulator to the radiator. This reduced linear model was developed in confunction with control studies for a thermionic reactor system (Weaver, Gronroos, Guppy, and Davis, 1969). These studies showed that such a reduction introduces only small errors in the values of the important variables being calculated.

The comparison, consequently, is between the linearized complete digital simulation and the linear reduced version of the analog simulation. The case of the $2 c$ step in reactivity was rerun with the linearized digital system and compared with the available corresponding analog results. The nonlinear digftal, the linear digital, and the linear (reduced version) analog results are compared in Figure 2.6. It may be observed that all results are now in 

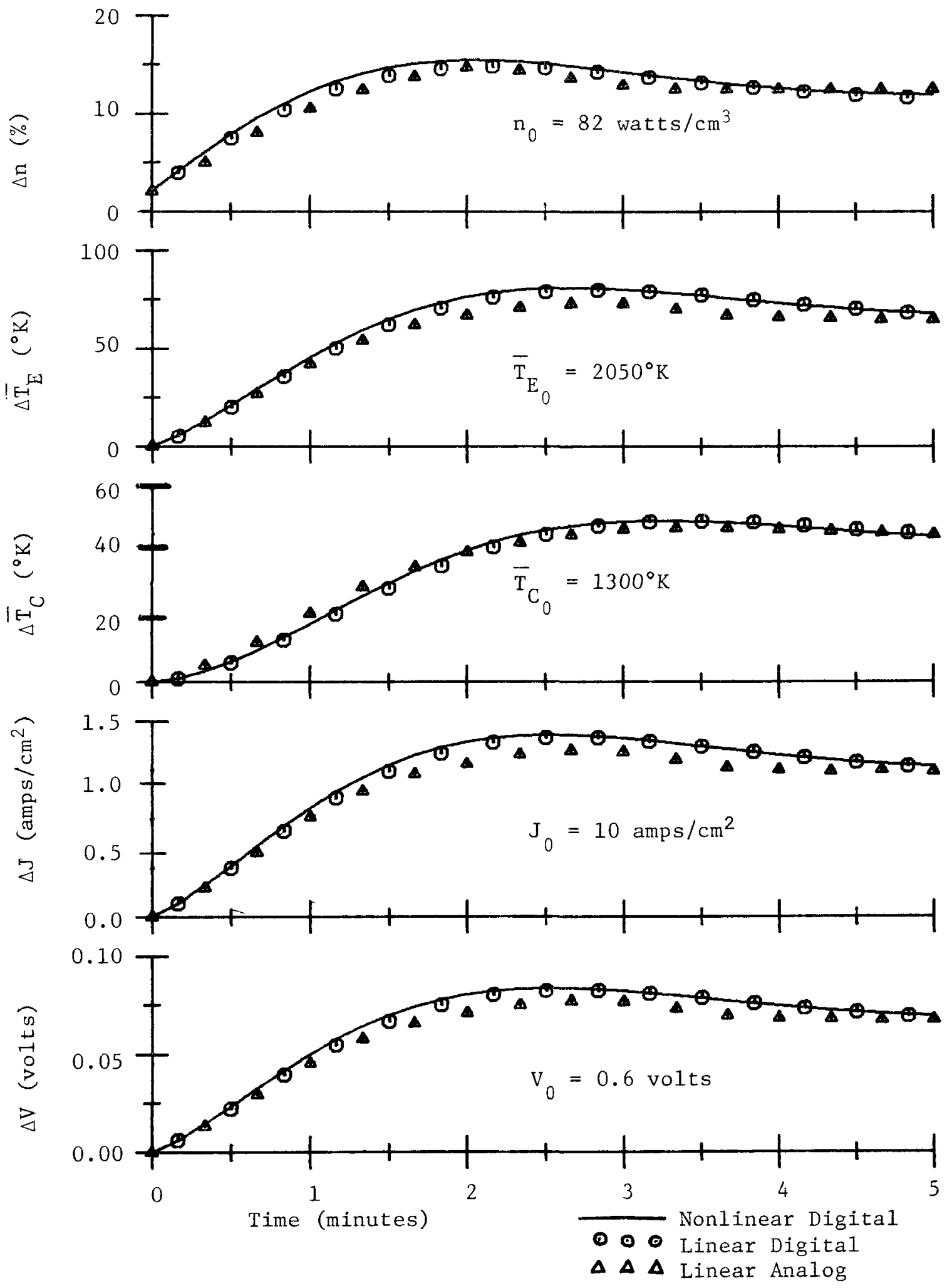

Figure 2.6. Comparisons of Nonlinear and Linear System Responses for +2 c Step 
excellent agreement. The nonlinear digital traces compare very closely both to the linear digital and the linear analog system responses as expected. The validity of using the reduced analog version for this comparison is also well demonstrated. The inaccuracies with the nonlinear analog results are thought to be attributable to electronic multiplier error.

Change in Load

With the reactor system again at equilibrium power, the resistive load is instantaneously decreased by $33 \%$. With the resistance lessened, electron cooling instantaneously increases, lowering the emitter temperature and raising the collector. No external control is applied to the reactor power so that it fluctuates slightly due to feedback effects until a new equilibrium level is attained. The nonlinear results are compared in Figure 2.7. The digital nonlinear response is compared to linearized digital and linearized analog system traces in Figure 2.8 .

The discrepancies between the nonlinear digital and nonlinear analog results are observed to be more serious than in the previous case. Also, the comparisons of the nonlinear digital and linearized models are not as close for this case. However, a $33 \%$ change in 1oad is a major perturbation and leads to the breakdown of the 1inearized equations describing the thermionic effects. Indeed, Figure 2.8 shows that the comparisons are close for the power and temperature curves but deteriorate for the current and voltage responses. 

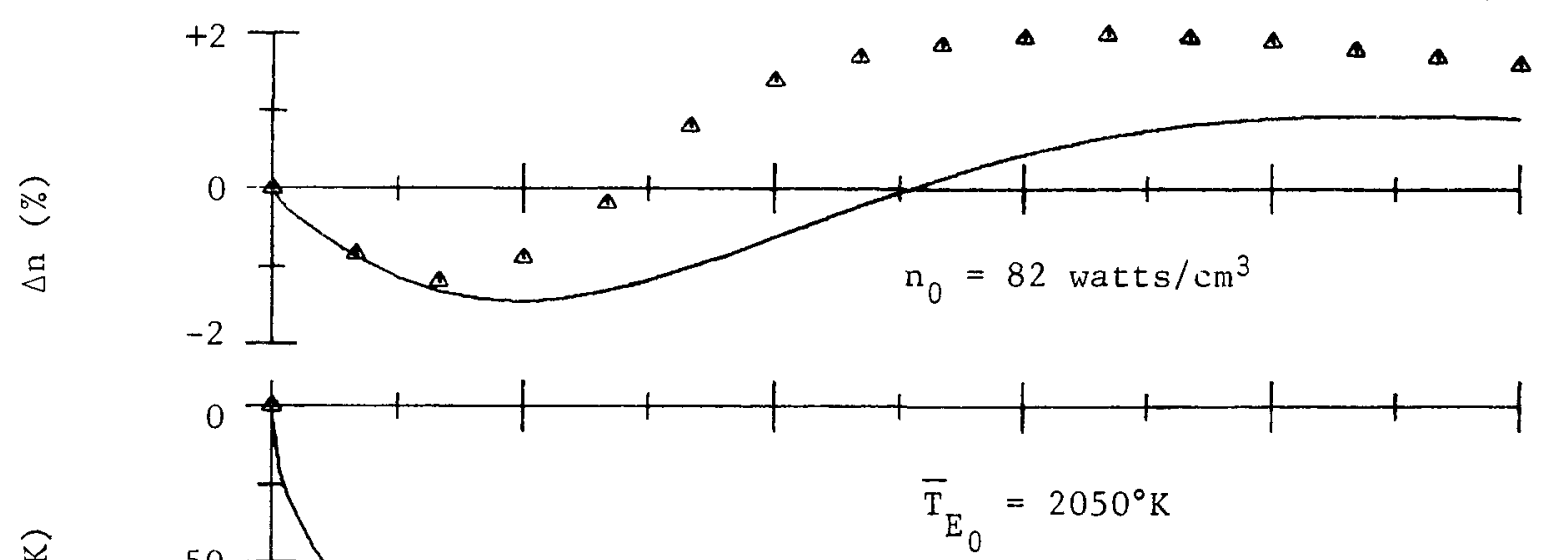

$\stackrel{\widehat{\vartheta}}{\mathcal{C}}$

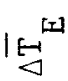

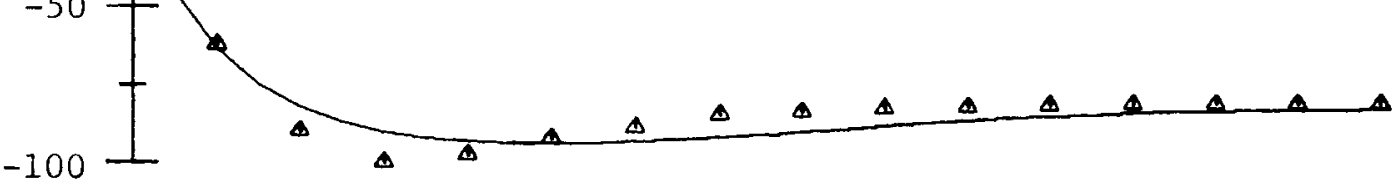

$\stackrel{2}{2}$

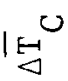

$\overline{\mathrm{T}}_{\mathrm{C}_{0}}=1300^{\circ} \mathrm{K}$

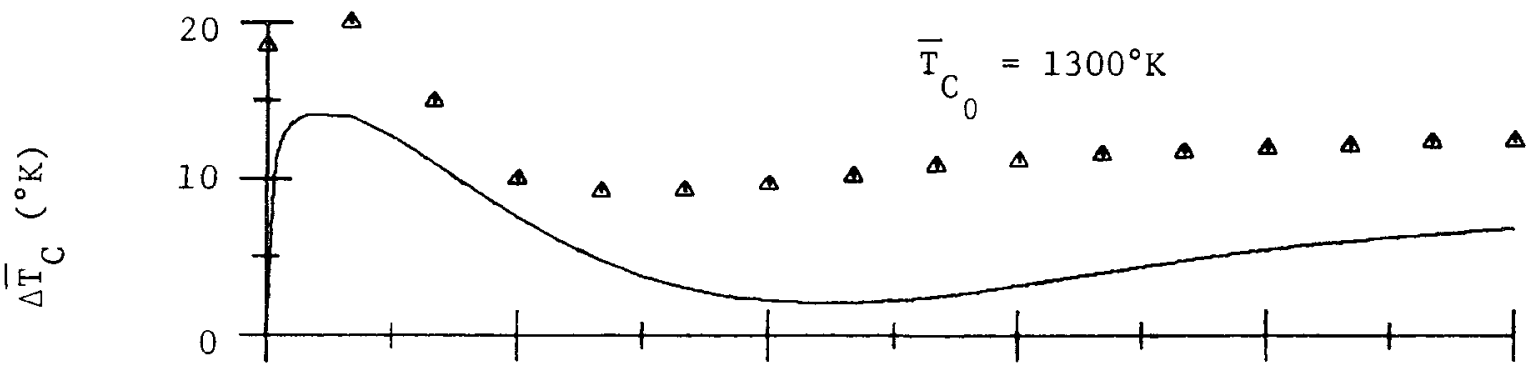

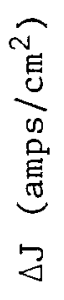

47

$\mathrm{J}_{0}=10 \mathrm{amps} / \mathrm{cm}^{2}$

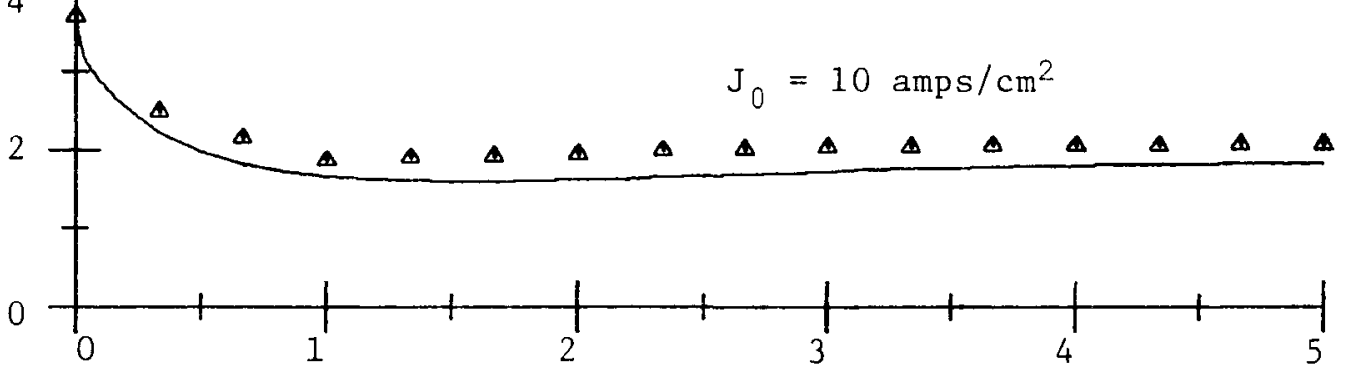

Time (minutes)

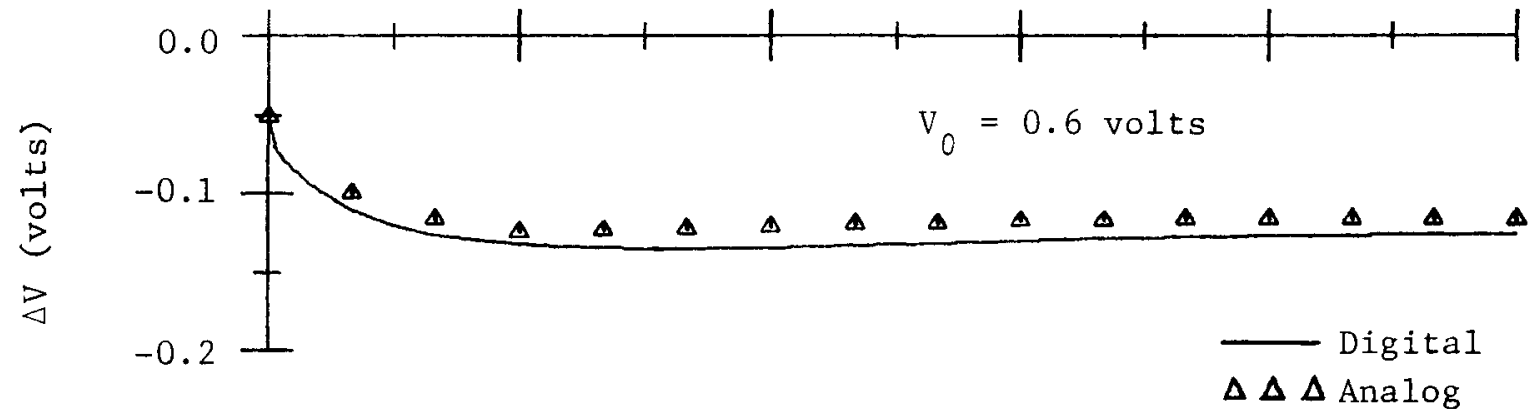

Figure 2.7. Comparison of Nonlinear System Response for Change in Load 

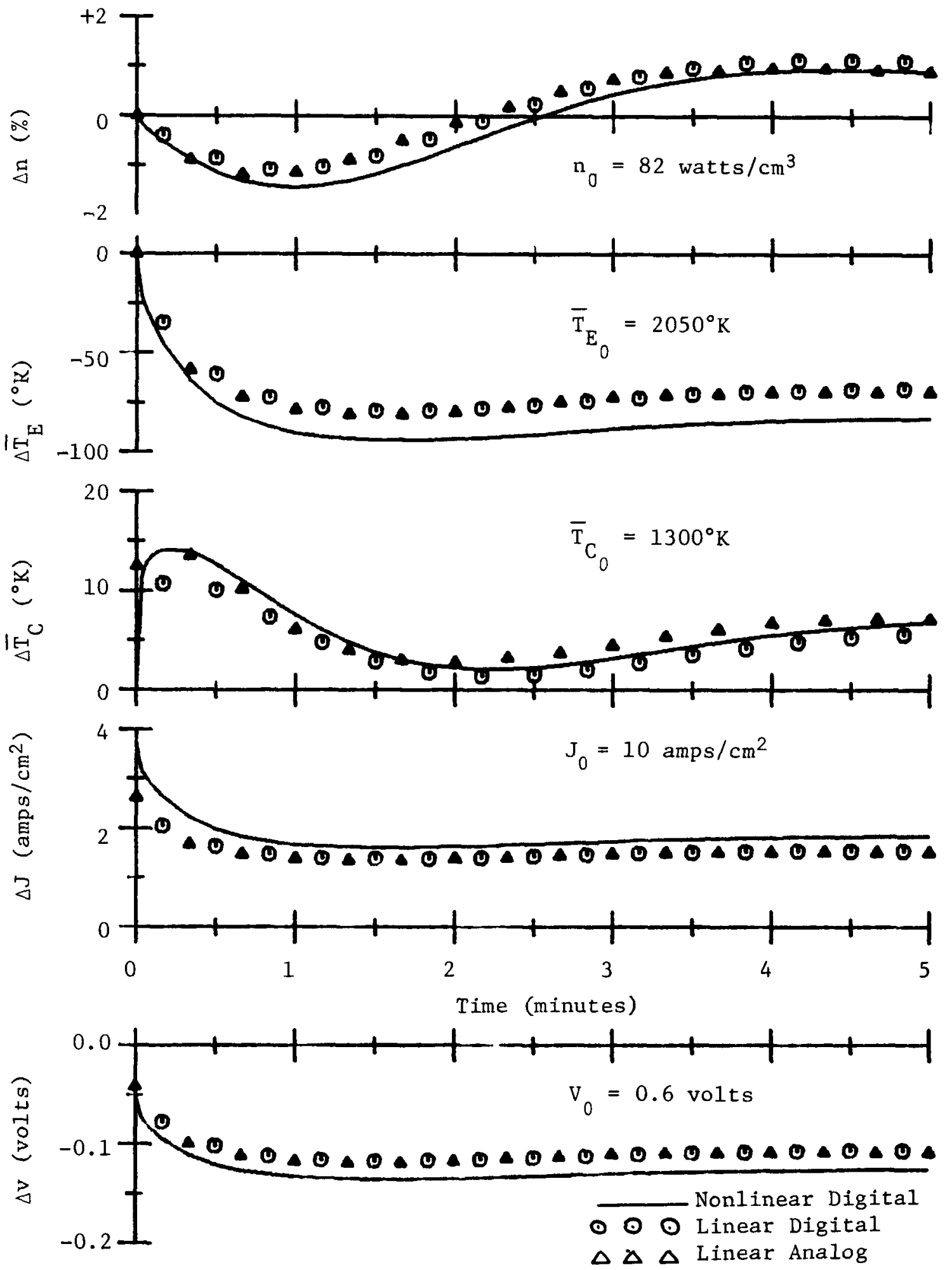

Figure 2.8. Comparisons of Nonlinear and Linear System Responses for Change in Load 
Discussion of Results

On the basis of the above comparisons, it is concluded that the reactor system describing equations are producing the correct response. Also, the digital integrator is capable of yielding accurate results. In addition, these studies appear to have indicated that the analog computer results for this highly nonlinear system may be in error.

The results of these typical transient cases point out the sluggish response characteristics of a thermionic reactor, as it takes several minutes for the system to stabilize following a perturbation. This behavior may be attributed to the various heat capacities and heat 1 oss rates as well as the small feedback coefficients inherent in this type of system. These results indicate that such a reactor should be relatively easy to control. 
CHAPTER III

THERMIONIC ANALYSIS

In this chapter description of the processes involved in thermionic converter operation is developed. The quantities of prime importance in system dynamic studies are the heat removed by the diode, the output current, and the output voltage. Various complex phenomena such as volume ionization, surface physics, and Schottky effects contribute to the determination of these quantities. The relative importance of these effects shifts as the diode operating conditions vary. Thus, any comprehensive model to describe converter performance over wide operating ranges must correctly account for these processes. Numerous theories have been developed while investigating the various aspects separately. Volume phenomena were studied by Hansen and Warner (1963a, 1963b, 1964), Shavit and Hatsopoulos (1964), Rasor (1965), Hansen (1965b), Warner (1965), and Wilkins and Gyftopoulos (1966a, 1967). Surface contributions were investigated by Rasor and Warner (1964), Levine and Gyftopoulos (1964), and Steiner and Gyftopoulos (1967). Schottky effects have been reported by Hansen (1965a, 1967). However, only Wilkins has successfully produced a computational model capable of representing a diode's performance characteristics over a wide range of operating variables. This model forms the basis of the digital computer code SIMCON (Wilkins, 1968), which is now in extensive use at several laboratories. 
SIMCON is utilized to calculate the steady-state parameters of interest in this study. The problem at hand, however, entails the description of a thermionic diode suitable for transient analysis. Using SIMCON as a starting point, work shown in this chapter extends this analysis to allow applications to a diode experiencing transients. Modifications to enhance convergence, as well as usage of this method with the system dynamic equations are discussed. The importance of proper initialization of certain critical parameters within the converter analysis is emphasized.

Finally, comparisons are drawn between this present model and the Richardson-Dushman approach for a diode description. Since this latter method is simpler, it is of interest to determine whether the more complex analysis developed here is indeed warranted to depict adequately the transient converter performance.

\section{Basic Thermionic Operation}

A thermionic converter consists essentially of two electrode surfaces: a high temperature emitter operating at around $2000^{\circ} \mathrm{K}$ and a lower temperature collector operating at about $1000^{\circ} \mathrm{K}$. The electrodes are separated by a spacing of several thousandths of an inch. A fraction of the emitter surface electrons escape from the surface, traverse the gap, enter the collector, and produce electrical power by returning to the emitter through an external load (see Fig. 3.1). When the diode is operated in a vacuum, this electron current becomes space charge limited at a very low value. However, if a gas (notably cesium vapor) is introduced into the interelectrode space, the diode efficiency can be greatly enhanced. In the case of cesium, some 


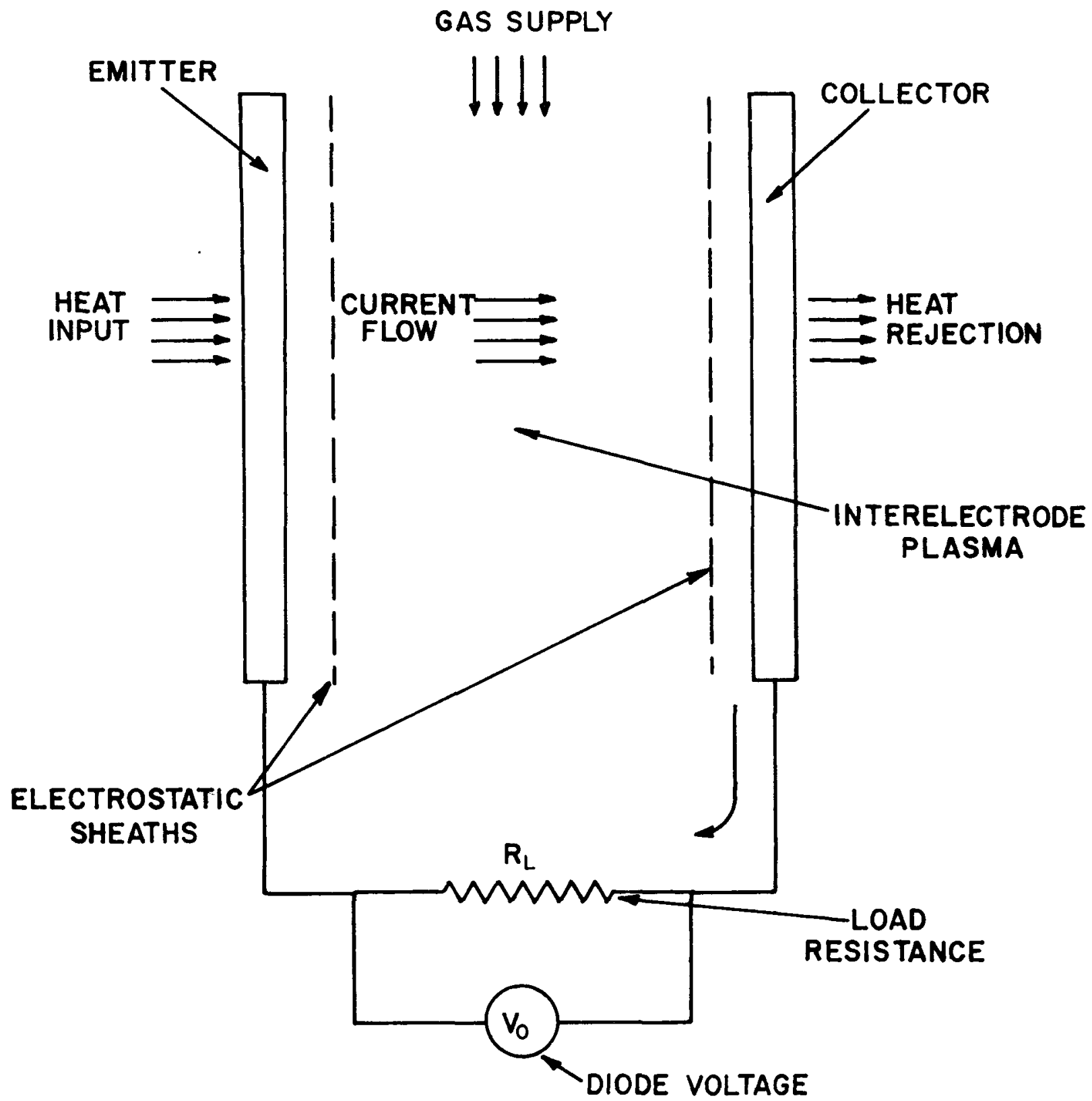

Figure 3.1. Basic Parameters and Processes Involved in Thermionic Converter Operation 
of the cesium atoms striking the hot surfaces become ionized. Also, electron-cesium collisions produce volume ionization in the gap. These positive ions will be attracted to the region of negative space charge and help neutralize it. If the cesium pressure in the converter is sufficiently high (approximately one to five torr), almost complete space charge neutralization can be achieved. This allows electron currents of appreciable magnitudes to traverse the gap.

In addition to this advantage, presence of cesium vapor results in two more gains. Gas atoms are adsorbed onto the emitter and collector surfaces. This causes the effective surface work functions to be lowered, since the work function for cesium is less than those of commonly used enitter and collector materials. The lowering of the emitter work function leads to increased electron emission currents from that surface. The lowering of the collector work function enhances the output voltage.

Since electrons collide with cesium atoms in the gap, some will be scattered back to the emitter. In spite of this, the high pressure cesiated thermionic converter has superior operating characteristics over non-cesiated types and can reach efficiencies of more than ten per cent.

These electron-cesium interactions further complicate any analytical model used to describe such a gas filled diode. Indeed, at cesium pressures and electron currents of practical interest, this effect is so great that almost the entire gap is collision dominated. The motion of ions and electrons becomes randomized and requires a plasma transport solution. 
Furthermore, the cesiated diode has the added complexity caused by the existence of electrostatic sheaths adjacent to the electrodes. These arise from variations in ion production near the surfaces, and they determine to a large extent the net current to or from the electrodes. These sheaths are thin compared to the diode spacing, but their presence must be accounted for.

Thus, the description of a gas filled thermionic diode requires the complex intermeshing of three main phenomena; i.e., interelectrode plasma, electrostatic sheaths, and surface effects.

\section{Development of Converter Analysis}

As stated, the only thermionic model developed to date which successfully incorporates all these requirements and is applicable over wide ranges of normal diode operation is the SIMCON digital code by Wilkins. The regime of validity of this analysis is listed in Table 3.1 .

The method requires the specification of the diode thermal conditions and certain known physical constants for the particular surface materials and interelectrode gas under study. It then calculates the converter output voltage and transport heat for known thermal conditions once a desired output current is selected.

The model also permits "adjustments" to be made to various internal mechanisms by changing certain input parameters. Thus, the results of the analysis can be "calibrated" to duplicate any given experimental data to a good degree of accuracy.

However, this method is designed to yield thermionic converter performance characteristics for a set of static thermal conditions and is not amenable as written to describe an operating diode experiencing 
TABLE 3.1

Ranges of Validity of Thermionic Analysis

\begin{tabular}{lr}
\multicolumn{1}{c}{ Parameter } & \multicolumn{1}{c}{ Range } \\
Emitter Temperature & $1650-2300^{\circ} \mathrm{K}$ \\
Collector Temperature & $700-1200^{\circ} \mathrm{K}$ \\
Cesium Temperature & $550-670^{\circ} \mathrm{K}$ \\
Output Current & Positive Values \\
Diode Spacing & $6-20$ mils
\end{tabular}

temperature or load transients. In this study, the SIMCON code is extended to allow application to diodes undergoing transients, and certain refinements are made to enhance its reliability. Before discussing these modifications, it is useful to understand the underlying principles and restrictions upon which SIMCON is formulated. As noted above, the proper description of a thermionic diode entails the inclusion of three main processes. The plasma analysis, as modeled by Wilkins and Gyftopoulos (1966b), considers a low energy, three component, two temperature plasma consisting of electrons, ions, and neutral particles. The electrostatic sheath analysis serves as a set of boundary conditions which allow solution to the plasma differential equations. The surface effects are treated separately, but must be correctly accounted for in the electrostatic sheath boundary conditions. The plasma analysis is discussed first.

Plasma Description

Describing equations must be written to represent the distribution of the particles of interest in the plasma. By initially 
assuming that neutral-electron and neutral-ion interactions occur much less frequently than neutral-neutral collisions, the distribution of neutral particles will be essentially unperturbed by motions of the charged particles. Thus, the velocity distribution of the monatomic neutrals is restricted to pure Maxwellian and describing equations need only be written for electrons and ions. If the electric field is the only external force present, the steady-state Boltzmann equations for electrons and ions are of the form

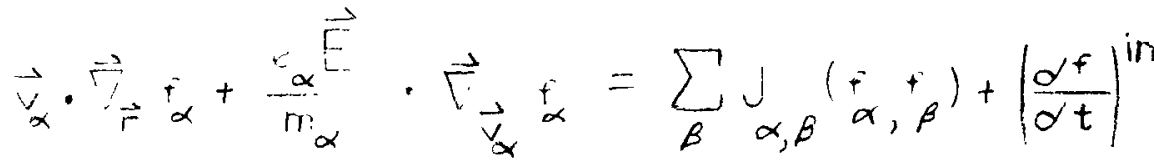

$$
\begin{aligned}
& \alpha=e, i ; \beta=e, i, 0
\end{aligned}
$$

where

$$
\begin{aligned}
\text { subscripts } & e=\text { electrons; } i=\text { ions; } 0=\text { neutrals } \\
e_{\alpha} & =\text { charge of species } \alpha \\
m_{\alpha} & =\text { mass of species } \alpha \\
\vec{v}_{\alpha} & =\text { velocity of species } \alpha \\
f_{\alpha}\left(\vec{r}_{,} \vec{v}_{\alpha}\right) & =\text { distribution function of species } \alpha \\
\overrightarrow{E_{w}}(\vec{r}) & =\text { electric field } \\
J_{\alpha, \beta}(f, f, \beta & =\text { elastic collision integral for interactions between } \\
\left(\frac{\partial f}{\partial t}\right)^{i n} & =\text { inelastic collision integral for species }
\end{aligned}
$$

From these two Boltzmann equations, the macroscopic mass, momentum, and energy conservation equations for each species may be obtained by multiplying each equation by $1, n_{\alpha} \vec{v}_{\alpha}$, and $m_{\alpha} v_{\alpha}^{2} / 2$ respectively and integrating each result over the $\alpha$-velocity space. 
A set of six equations is obtained which may be solved once the charged particle distribution functions ( $f_{k}$ and $f_{i}$ ) are known. The two original Boltzmann equations are used to determine these distribution functions. However, before this is accomplished, several valid simplifying assumptions are made which allow explicit solutions of these equations.

Solution of Boltzmann Equations. For collision dominated plasmas, the charged particle distribution functions are nearly Maxwellian. Thus, solutions may be sought of the form

$$
{ }_{\alpha}\left(\vec{r}, \vec{r}_{\alpha}\right)=f_{\alpha}^{0}\left(\vec{r}, \vec{v}_{\alpha}\right)\left[1+\phi_{\alpha}\left(\vec{r}, \vec{v}_{\alpha}\right) ;|\phi|<1\right.
$$

where:

$$
f_{\alpha}^{0}\left(\vec{r}_{,} v_{\alpha}\right)=r_{\alpha}\left(\begin{array}{ll}
r_{\alpha} \\
\hdashline \pi K_{E} T_{\alpha}
\end{array}\right)^{2} \operatorname{exk}\left(\begin{array}{c}
-m_{\alpha} v_{\alpha} \\
\left.2 K_{\beta}\right]_{\alpha}
\end{array}\right)
$$

and

$$
\alpha=e, i
$$

$$
\begin{aligned}
n_{\alpha}(\vec{r}) & =\text { particle density of species } \alpha \\
T_{\alpha} & =\text { temperature of species } \alpha \\
K_{F} & =\text { Boltzmann constant }
\end{aligned}
$$

This simplification permits linearization of the nonlinear Boltzmann equations.

When only short range, two body interactions are considered, the elastic collision integrals $J_{\alpha, \beta}\left(f_{\alpha}, f_{f}\right)$ may be written in a special Boltzmann form (Chapman and Cowling, 1961). If the further assumption is made that $m_{e} / m_{\beta}<<(\beta=i, 0)$, these integrals may be subsequently reduced with the end result of decoupling the electron and 
ion Boltzmann equations in velocity space. Also, the electron-ion and electron-neutral elastic energy terms in the macroscopic conservation equations reduce to zero from this condition.

Due to multiple collisions between ions and neutrals, the temperature of these heavy particles may be assumed to be the same, i.e., $T_{i}=T_{0}$. It is therefore unnecessary to retain the macroscopic ion energy conservation equation.

If consideration is restricted to low energy plasmas, a requirement which thermionic diodes nearly fulfill, the inelastic collision integrals, $\left(\alpha_{\alpha}^{\prime} / \alpha t\right)^{i n}$, may be neglected, since these effects occur predominantly at higher particle energies. This condition simplifies both Equations (3-1). However, the inelastic source terms in the macroscopic mass conservation equations and the inelastic energy transfer in the macroscopic electron energy equation are still included, since their corresponding elastic terms are already zero.

With these conditions, the two Boltzmann Equations (3-1) may be solved for $\phi\left(\vec{r}, \vec{v}_{\alpha}\right)(\alpha=e, i)$. Solutions obtained are in terms of the complete, orthonormal, Sonine polynomial expansions of order $3 / 2$ (Chapman and Cowling, 1961). As an outgrowth of these solutions, the distribution functions so determined satisfy the macroscopic momentum conservation equations. Consequently, these momentum equations do not require further consideration. The remaining macroscopic equations to be computed have thus been reduced from six to three, the ion energy conservation equation being neglected since $T_{i}=T_{j}$.

Solution of Macroscopic Equations. Before these macroscopic equations are written in their final form, three additional assumptions 
are made so that an explicit solution to these differential equations may be found. Two of these restrictions are generally acceptable, but the third compromises the validity of the model somewhat. However, without this third assumption, the resulting equations may not be directly integrated.

The first assumption states that spatial variations of plasma properties occur in one dimension only. Secondly, the charged particle densities of the electrons and ions in the plasma are assumed to be equal. This fact is not valid in the electrostatic sheaths where the electric field is strong, but in the plasma away from the sheaths, charge neutrality is very nearly preserved. An outgrowth of this assumption is that the net current density across the plasma is a constant and may be expressed

$$
J=J_{e}(x)-J_{i}\left(x_{i}=\right.\text { constant }
$$

where

$$
\begin{aligned}
J & =\text { net current density } \\
J_{e} & =\text { spatially varying electron current } \\
J_{i} & =\text { spatially varying ion current }
\end{aligned}
$$

The third assumption is that spatial variattons of the electron and ion temperatures, as well as those of transport coefficients which appear in the plasma differential equations may be neglected. This constant particle temperature approximation can result in errors when computing such terms as volume ionization, recombination, or boundary conditions, since these are temperature dependent. However, 
predictions by SIMCON to such bulk diode characteristics as current and output voltage have been shown to be of sufficient accuracy to make these restrictions acceptable (Wilkins, 1968). If detailed determination of spatially varying interelectrode processes is desired, the plasma analysis becomes much more complex.

Thus, the remaining three macroscopic conservation equations may be written

$$
\begin{aligned}
& \frac{d U_{e}}{d x}=\frac{d U_{i}}{d x}=e \nu_{i} n_{p}-\beta_{r} n_{p}^{3} \\
& \frac{d q_{e}}{d x}=-J \cdot E-V_{i} \cdot \frac{d U_{i}}{d x}
\end{aligned}
$$

where

$$
\begin{aligned}
q_{e}(x) & =\text { electron kinetic energy flux } \\
n_{p}(x) & =\text { charged particle density } \\
V_{i} & =\text { ionization frequency } \\
\beta_{r} & =\text { three-body recombination coefficient } \\
V_{i} & =\text { ionization potential of interelectrode gas }
\end{aligned}
$$

Terms in the right-hand side of Equations (3-5) describe the inelastic particle source rate for mass conservation, while terms in (3-6) represent the rate of inelastic energy production.

These differentiated quantities (i.e., $J_{e}, J_{i}$, and $q_{e}$ ) also have equations resulting from their respective definitions when the macroscopic properties are first formulated from the distribution functions. The solutions to these definitions are expressed in terms of 
the distribution functions explicitly as

$$
\begin{aligned}
& J_{e}(x) \equiv e \int v_{e} f_{e}\left(x, v_{e}\right) d v_{e}=-e \mu_{e}\left[\theta_{e} \frac{d n_{p}}{d x}+n_{p} E\right] \\
& J_{i}(x) \equiv e \int v_{i} f_{i}\left(x, v_{i}\right) d v_{i}=-e \mu_{i}\left[\theta_{i} \frac{d n_{p}}{d x}-n_{p} E\right] \\
& q_{e}(x) \equiv \int \frac{n_{e} v_{e}}{2} f_{e}\left(x, v_{e}\right) d v_{e}=v_{e}\left[\frac{3}{2}+y_{e}\right] \theta_{e}
\end{aligned}
$$

where

$$
\begin{aligned}
& \mu_{\alpha}=\text { mobility of species } \alpha \\
& \Theta_{\alpha}=\text { voltage equivalent temperature of species } \alpha \\
& \gamma_{e}=\text { electron thermoelectric coefficient }
\end{aligned}
$$

All the various transport coefficients in Equations (3-5) to (3-9) are assumed spatially independent and have definite values in terms of either polynomial expansions or the processes which they describe. The spatially varying quantities to be determined are $J_{e}$, $J_{i}, q_{e}, n_{p}$, and $E$.

Equations (3-5) may be combined with the derivatives of (3-7)

and (3-8) to eliminate $E$ and arrive at the ambipolar diffusion equation in terms of $n_{p}$ only. By making several changes, this may be put in the dimensionless form

$$
\frac{d^{2} y}{d \epsilon^{2}}=-2 y^{2} y\left(1-y^{2}\right)
$$


where

$$
\begin{aligned}
y(\epsilon) & \equiv n_{p} / n_{s} \\
\epsilon & \equiv x / d \\
n_{s} & =\text { Saha equilibrium charged particle density } \\
d & =\text { interelectrode spacing } \\
\nu_{i} / \beta_{r} & =n_{s}^{2} \\
\gamma^{2} & \equiv \nu_{i} d^{2} /\left(2 U_{a}\right) \\
D_{a} & \equiv \mu_{e} \mu_{i}\left(\theta_{e}+\theta_{i}\right) /\left(\mu_{e}+\mu_{i}\right)
\end{aligned}
$$

The ambipolar diffusion equation is integrable and yields solutions involving Jacobi elliptic functions (Abramowitz and Stegun, 1965).

There are two integration constants resulting from the solution of $y(\in)$. The most important (denoted $K$ ) comes from the first integral of $(3-10)$

$$
\frac{d y}{d \epsilon}= \pm \gamma \sqrt{K-y^{2}\left(2-y^{2}\right)}
$$

The magnitude of $K$ determines which Jacobi elliptic function form that $y(\epsilon)$ will take. Furthermore, when $K$ nears unity (corresponding to $Y>5$ ), asymptotic expansions of the elliptic functions may be used to simplify the analysis. Thus, there are three possible solutions to $y(\epsilon)$, the appropriate one being set by the converter operating conditions. In any event, a definite solution to $n_{p}$ may be obtained, subject to boundary conditions.

The electric field may be determined from Equations (3-7) and $(3-8)$ 


$$
E(x)=-\frac{\mu_{e} \theta_{e}-\mu_{i} \theta_{1}}{y\left(\mu_{e}+\mu_{i}\right) d} \cdot \frac{d y}{d \epsilon}-\frac{J_{e}-J_{i}}{e\left(\mu_{e}+\mu_{i}\right) n_{s} y}
$$

This may be integrated to yield the electrostatic potential profile in the interelectrode plasma, subject to boundary conditions. To understand adequately the final technique used to solve the problem now posed, a discussion of the remaining two areas of thermionic diode description (i.e., electrostatic sheaths and surface effects) is necessarv.

Sheath Analysis

The sheath analysis is included as a set of boundary conditions at the electrode surface-plasma interface. This is accomplished by writing electron and ion current and electron kinetic energy flux (i.e., $J_{e}, J_{i}$, and $q_{e}$ ) balances across the sheaths. This yields three boundary conditions at the emitter edge of the plasma and three similar ones at the collector.

These boundary conditions, however, may assume one of two forms depending upon the polarity of the sheath in question. A sheath is said to be accelerating (negative voltage change) if it speeds up an electron traveling toward the collector (see Fjg. 3.2). Similarly, a sheath is retarding (positive voltage change) if it slows an electron traveling toward the collector. In Figure 3.2, the emitter sheath is accelerating $\left(V_{E}<0\right)$ while the collector sheath is retarding $\left(V_{C}>0\right)$. To simplify the analysis, the sheaths are assumed to be either monotonically increasing or decreasing. 

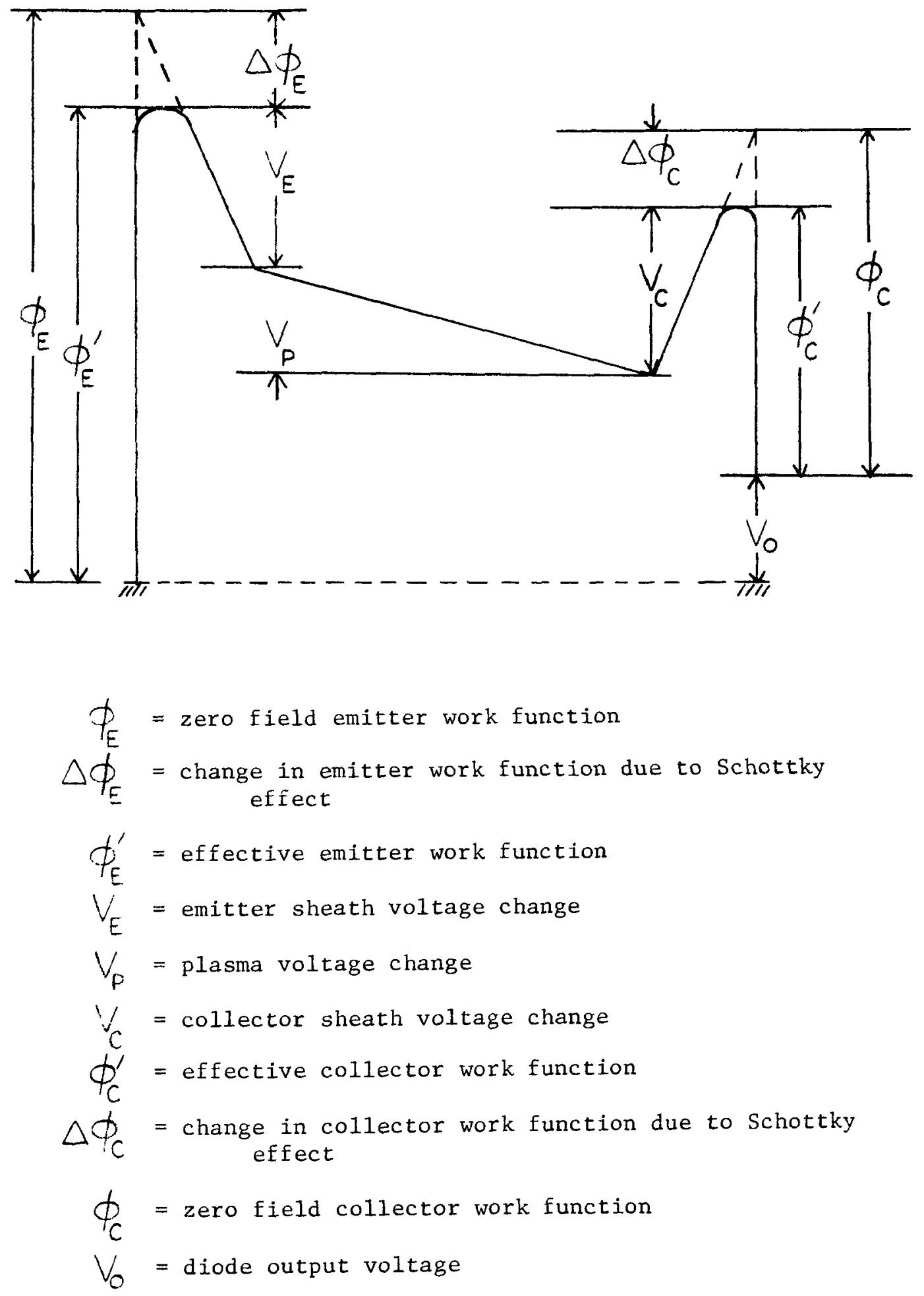

Figure 3.2. Typical Voltage Profile Across Thermionic Diode 
Thus, for any given combination of sheath polarities, six boundary conditions may be supplied. Two are required to determine these sheath voltage changes $\left(V_{E}\right.$ and $V_{C}$ in Fig. 3.2), while the remaining four may be used in specifying $J_{e}, q_{e}, n_{p}$, and $E\left(J_{i}\right.$ being related to $J_{e}$ through Eq. (3-4)).

Surface Analysis

However expressed, these boundary conditions couple the surface electron and ion emission current densities to the plasma electron and ion current densities and the electron kinetic energy flux (see Fig. 3.3). As mentioned earlier, these surface phenomena may be dealt with separately. Also, the emitter surface effects are divorced from those of the collector.

Work Function Determination. Knowing only the surface temperature, interelectrode vapor pressure, and certain physical constants of the material and gas under study, the field-free electron and ion emission current densities may be determined. These current densities follow directly from the Richardson-Dushman equation for electrons, and the Saha-Langmuir equation for ions. They are of the form

$$
\begin{aligned}
& J_{j}=A T_{j}^{2} \exp \left(-\phi_{j} / \theta_{j}\right)
\end{aligned}
$$

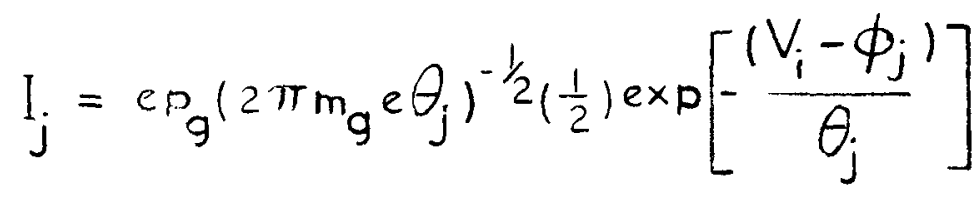

where 


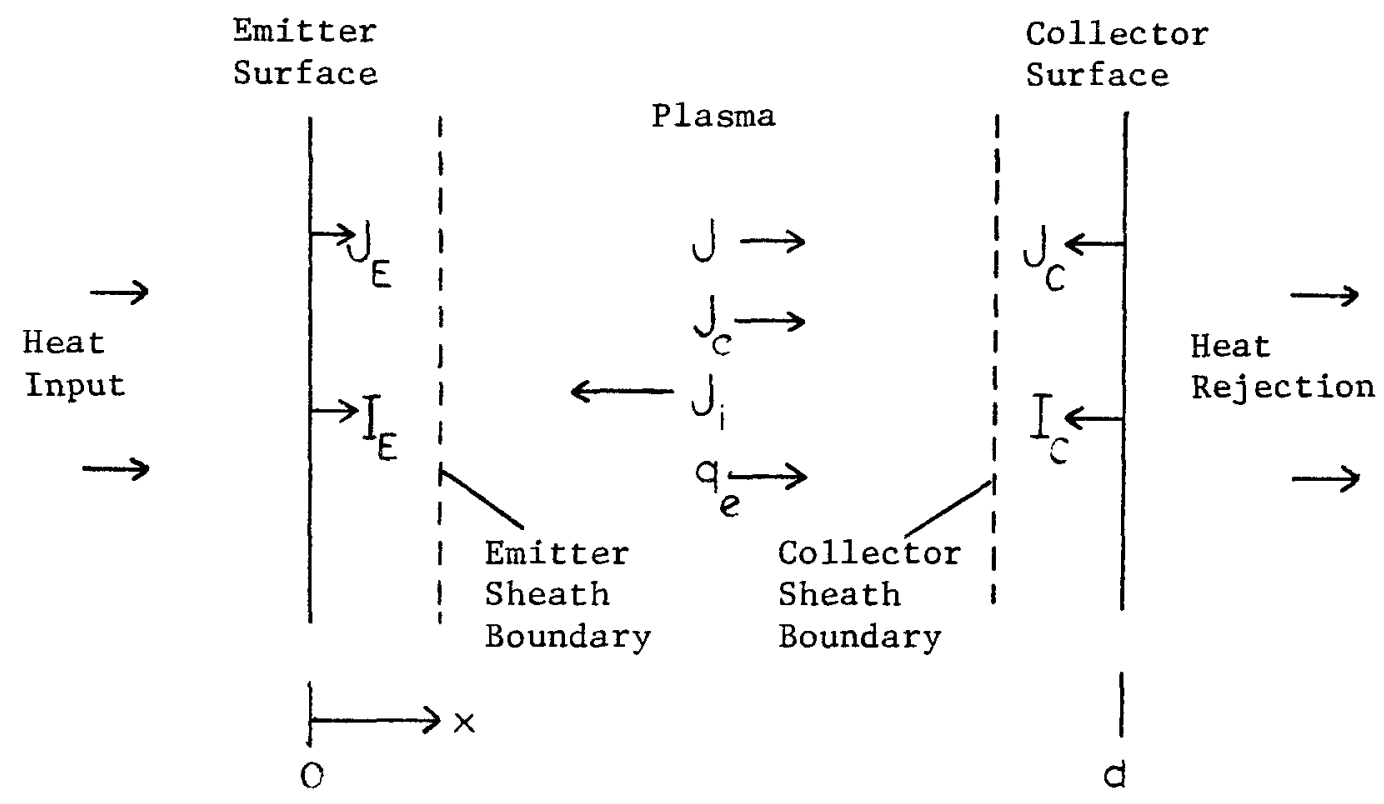

$J_{E}=$ emitter surface electron emission current
$I_{E}=$ emitter surface ion emission current
$J_{C}=$ collector surface electron emission current
$I_{C}=$ collector surface ion emission current
$J_{e}=$ electron current in plasma
$J_{i}=$ ion current in plasma
$J=$ net converter current density
$q_{e}=$ electron kinetic energy flux

Figure 3.3. Relation of Surface and Plasma Current Densities 


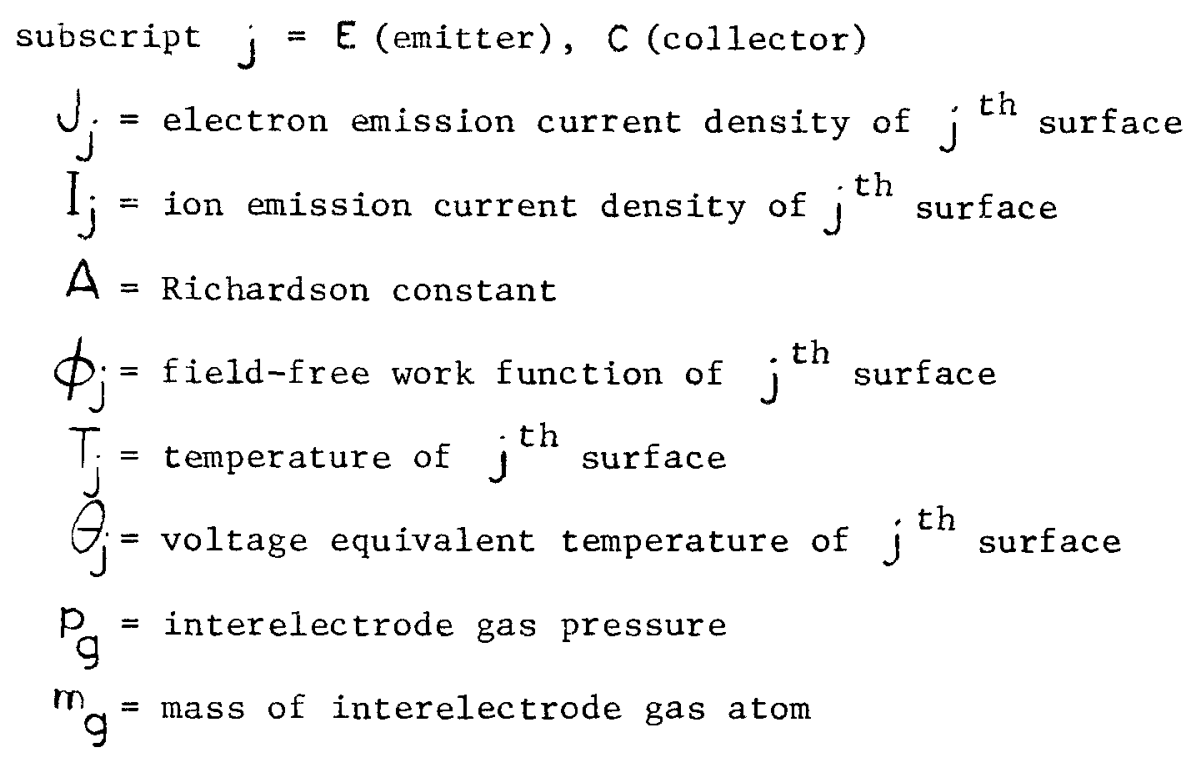

A11 terms in Equations (3-13) and (3-14) are known a priori except $\phi_{j}$. Wilkins follows the analysis developed by Steiner and Gyftopoulos (1967) to determine these field-free surface work functions $\left(\phi_{E}\right.$ and $\phi_{C}$ in Fig. 3.2). This method permits an iterative solution which involves balancing the arrival and departure rates of heavy particles at the electrode surface. Thus, the field-free electrode work function in the presence of an interelectrode gas can be computed, and the corresponding surface emission current densities may be set.

Schottky Effects. One last property which must be included prior to the solution of the plasma differential equations is the Schottky effect on the surface work functions. This effect is caused by the presence of an imposed electric field, in this case the field due to the electrostatic sheath. Consequently, the electrode work function will experience an effective change (see Fig. 3.2). Wilkins models this Schottky effect in terms of the corresponding sheath voltage change. Thus, this correction may be determined simultaneously 
with the boundary condition calculations. It involves multiplying the appropriate ion and electron emission current densities by an additional exponential factor.

Mathematically, the problem is now ready to be solved. However, the actual numerical solution requires a complex iterative procedure.

Numerical Method of Solution

The objective, as modeled by Wilkins, is to calculate the thermionic converter output voltage ( $V_{0}$ in Fig. 3.2) for a given set of operating conditions. This operating point is determined by the quantities $T_{E}, T_{C}, T_{R}, J$, and $d$ where

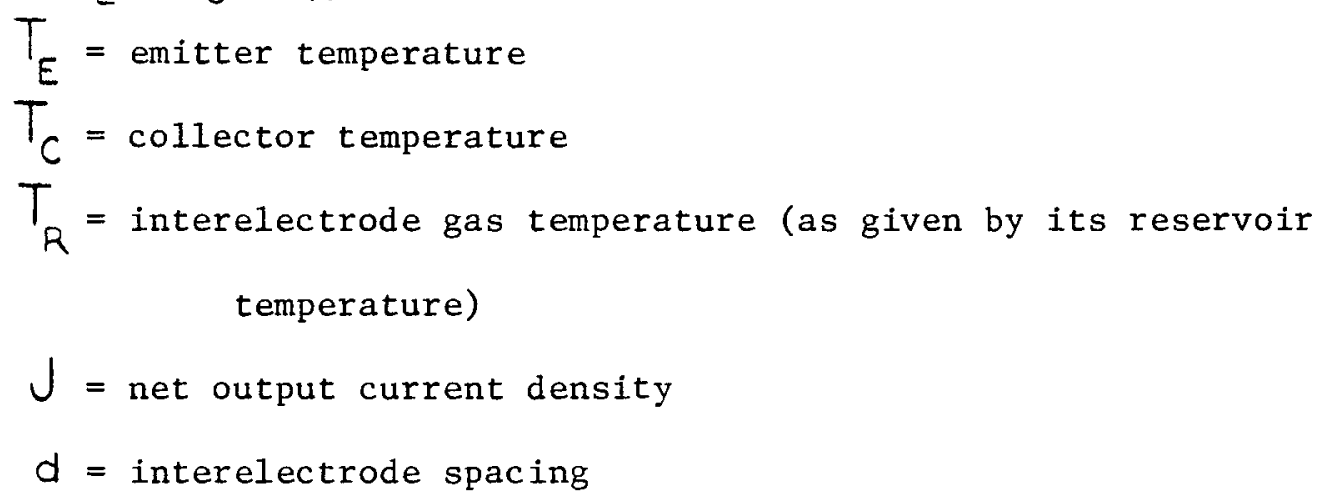

The output voltage is set by

$$
V_{O}=\phi_{E}^{\prime}+V_{E}+V_{P}+V_{C}-\phi_{C}^{\prime}
$$

(see Fig. 3.2). All quantities in the Equation (3-15) may be calculated for the specified thermal conditions once the plasma differential equations, sheath boundary conditions, and surface contributions are determined. The iterative numerical scheme used is now briefly discussed. 
From knowledge of the respective surface and reservoir temperatures, as well as certain input data, the emitter and collector electron and ion emission current densities $\left(J_{E}, J_{C}, I_{E}\right.$, and $\left.I_{C}\right)$ plus the field-free surface work functions $\left(\phi_{E}\right.$ and $\phi_{C}$ ) may be initially set. With the specification of the desired net output current $(J)$, the plasma differential equations can be solved.

Although the ion temperature $\left(T_{i}\right)$ and its voltage equivalent $\left(\theta_{i}\right.$ ) may be expressed in terms of the known surface temperatures, the electron temperature $\left(T_{e}\right.$ or $\left.\theta_{e}\right)$ is not so easily determined. An initial value must be selected so that the solution can proceed. Then, the ambipolar diffusion equation may be integrated to calculate $n_{p}$. Since the sheath boundary conditions are involved in the solution to this plasma differential equation, the determination of $n_{p}$ also yields $J_{e}, J_{i}, q_{e}, V_{e}$, and $\phi_{E}^{\prime}$ at the emitter edge of the plasma, plus $J_{e}, J_{i}, q_{e}, V_{c}$, and $\phi_{c}^{\prime}$ on the collector side.

The electric field equation, (3-12), may now be integrated to set the plasma voltage change $\left(V_{p}\right.$ in Fig. 3.2) since

$$
V_{p} \equiv \int_{0}^{d} E(x) d x
$$

Thus, all terms in the right-hand side of (3-15) are known. Consequently, the diode output voltage for the assumed electron temperature value and the specified converter operating conditions may be computed. However, since the original estimate of the electron temperature may be in error, a check is required. This is accomplished through the use of Equation (3-6) in conjunction with the results of 
the sheath boundary conditions. Integration of (3-6), along with the use of (3-16) and (3-4), produces

$$
q_{e}(x)-a_{e}\left(01=-J \cdot J_{p}-V_{i}\left[J_{i}(d)-J_{i}(0)\right]\right.
$$

If this energy balance is not satisfied, the electron temperature must be corrected and the solution to the various plasma and sheath equations repeated until the conditions of (3-17) are met to within some specified error allowance.

Thus, for a specified converter operating point, the diode output voltage has been determined for a given output current. The other important parameter determined by this analysis is the total heat transported across the diode. This quantity (denoted $q_{E}$ ") is divided into radiation, cesium conduction, electron cooling, and ion cooling contributions and may be written

$$
q_{E}^{\prime \prime}=Q_{r}+r_{c s}+\dot{z}_{e}+\hat{r}_{i}
$$

where

$$
\begin{aligned}
& Q_{r}=\sigma\left[\frac{T_{E}^{4}}{2 E_{E}\left(T_{E}\right)+1 / \epsilon_{C}\left(T_{E} T_{C}\right)-1}-\frac{T_{C}^{4}}{V_{\epsilon_{E}}\left(T_{E, C} T_{C}\right)+1 / \epsilon_{C}\left(T_{C}\right)-1}\right] \\
& Q_{C S}=\frac{1.10 \times 10^{-6}\left(T_{E}^{\frac{3}{2}}-T_{C}^{\frac{3}{2}}\right)}{d+1.15 \times 10^{-5}\left(T_{E}+T_{C}\right) / P_{g}} \\
& Q_{e}=J_{e}(0)\left(\phi_{E}^{\prime}+V_{E}\right)+q_{e}(0) \\
& Q_{i}=-J_{i}(0)\left(\phi_{E}^{\prime}+V_{E}\right)+J_{i}(0) V_{1}
\end{aligned}
$$


and

$$
\begin{aligned}
& \sigma=\text { Stefan-Boltzmann constant } \\
& \epsilon_{E}=\text { temperature dependent emitter emissivity } \\
& \epsilon_{C}=\text { temperature dependent collector emissivity }
\end{aligned}
$$

With constant surface and gas temperatures, (3-19) and (3-20) are invariant. The electron and ion cooling contributions $((3-21)$ and (3-22) respectively) vary with current and voltage and must be evaluated with every converter calculation. The cesium conduction term, (3-20), is obtained from the analysis developed by Kitrilakis and Meeker (1963).

The prime function of SIMCON as written by Wilkins is to vary $J$ and calculate $V_{0}$ while holding the converter thermal conditions constant. When the resulting points are plotted, a typical currentvoltage $(I-V)$ curve is generated as shown in Figure 3.4. While such information is important when analyzing steady-state thermionic converter performance, it is not applicable as such to a diode undergoing a transient. However, work in the next section shows how this transient analysis may be accomplished.

\section{Extension to Transient Analysis}

As shown in Chapter II, the thermionic quantity of prime interest for transient thermal analysis is the emitter heat flux, $q_{E}^{\prime \prime}$. The determination of this, however, requires specification of the diode current and voltage profile (see Eqs. (3-18) to (3-22)). While the SIMCON analysis calculates these various parameters, an additional 


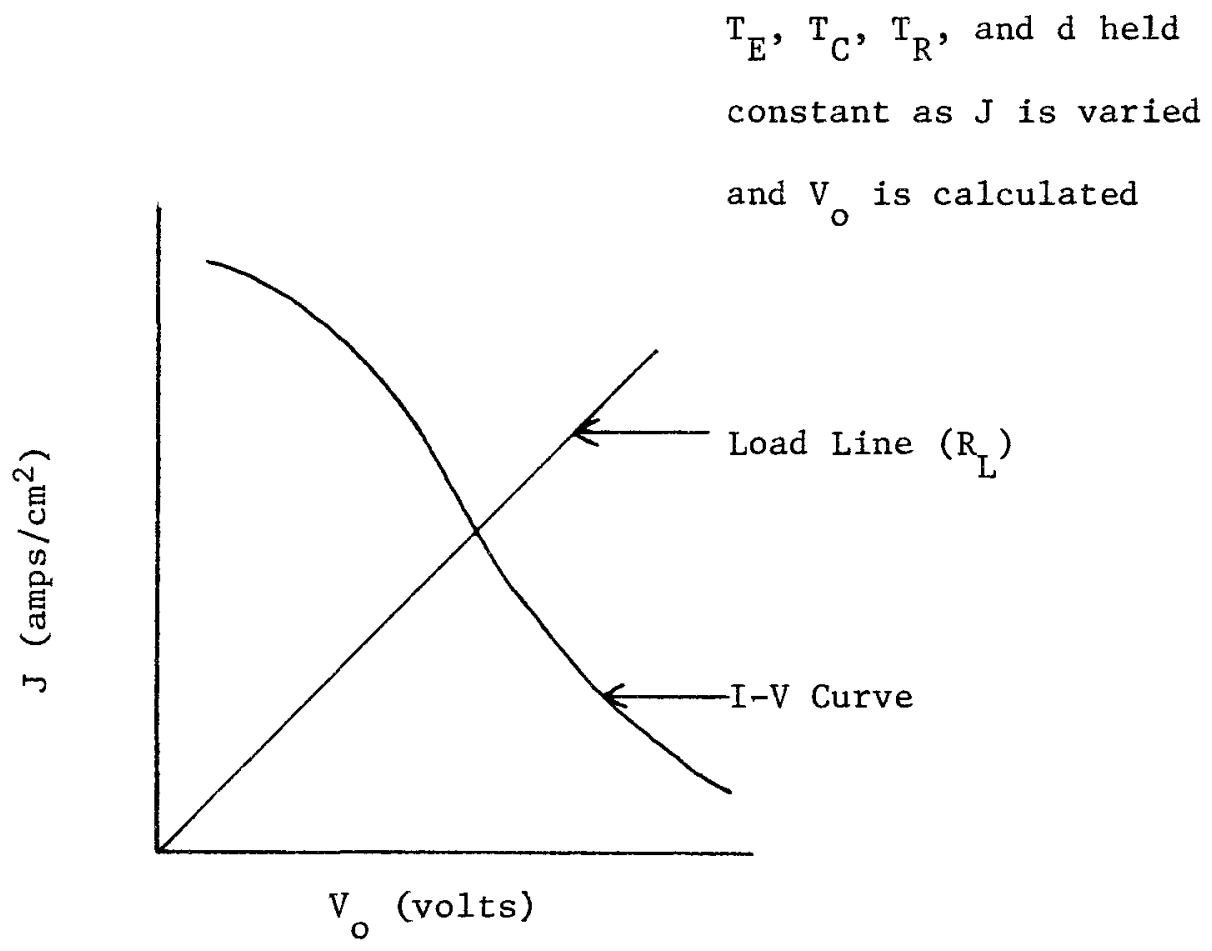

Figure 3.4. Typical Steady-State I-V Curve With Superimposed Load Line 
condition is needed to pinpoint the proper values for transient studies.

Any operating converter will be working across some resistive load. The output voltage is then linearly related to the output current through Ohm's Law

$$
V_{0}=J \cdot R_{L}
$$

where $R_{1}$ is the load resistance. A typical load line is superimposed on the $I-V$ plot shown in Figure 3.4. Thus, this added relationship permits the unique determination of the operating point on any $I-V$ curve.

Incorporation of this load line criterion into the SIMCON code analysis is discussed next. Then, additional modifications made to enhance convergence are noted.

Inclusion of the Load Line

The incorporation of the load line condition into the SIMCON program necessitates an additional iteration on the net output current density (J). The technique is analogous to shifting the current along the appropriate I-V curve as depicted in Figure 3.4. Thus, as with the electron temperature, a value is selected for $J$ and the plasma equations are solved for the corresponding output voltage. Knowing the load resistance, Equation (3-23) is then tested. If the condition is not satisfied, $J$ is incremented and the procedure repeated until the specifications are met. When Eq. (3-23) is satisfied, the values of 
J, $V_{\supset}$, and $q_{E}^{\prime \prime}$ are consequently pinpointed. The required set of diode conditions to determine the thermionic parameters of interest for transient analysis becomes $T_{E}, T_{C}, T_{A}, d$, and $R_{L}$. The variables $T_{E}$ and $T_{C}$ are given by their corresponding thermal describing equations. $T_{R}, d$, and $R_{L}$ are specified separately, but may be time varying. Thus, the SIMCON program, modified to include the given load resistance, can now yield the proper diode current. voltage, and emitter heat flux values as the emitter and collector temperatures fluctuate.

Further discussion of this additional iteration on diode current is contained in the next section concerning convergence. The proper convergence of the thermionic analysis constitutes an important segment of its application to transient studies.

Enhancement of Convergence

Since the entire thermionic analysis deals with an array of nonlinear equations, the calculation of many other pertinent parameters, as well as current and electron temperature, requires an iterative procedure. Inherent with any iterative process is the possibility of convergence failure. When this repetitive thermionic scheme is coupled with the transient system equations, multiple use of the analysis is necessary. To minimize the possibility of nonconvergence, several internal refinements are made in the plasma analysis.

Specific Modifications Incorporated. The important parameters to which iterative modifications are applied include diode current 
$(J)$, electron temperature $\left(T_{e}\right)$, dimensionless plasma charged particle density $(y)$ evaluated at both sheath boundaries, and the sheath voltages $\left(V_{E}, V_{C}\right)$. These critical variables are not allowed to fluctuate beyond set deviation limits from iteration to iteration. This insures smooth transitions as the particular converged solution is sought. The specific deviation limits used in this study are noted in the program listings contained in the appendix. While their values are flexible, the limits indicated have proven from experience to be reasonable.

Also, as previously mentioned, solutions to the ambipolar diffusion equation may assume any of three forms as determined by the integration constant $K$ and parameter $Y$. A separate analysis is required for each form. Since $K$ is not known a priori, a nonconvergence can occur when the cross-over points are approached. Thus, whenever such a failure is detected, an added check is made to see whether the solution lies near a cross-over point. If it does, the calculations are diverted to the proper analysis, and the solution continues, Otherwise, the problem terminates.

All iterations are checked by an iteration counter. If the specified maximum number is exceeded, a nonconvergence has occurred and a proper indication is made. However, since solutions always exist if the specified diode conditions are valid (see Table 3.1), a further check is made before the case is aborted. Provided the variable in question is known to within a specification of two or three times normal, the solution is allowed to proceed. If, however, the error is 
too gross, the problem terminates. It should be mentioned here that in all transient cases attempted during this study, the thermionic analysis never failed to reach a converged solution, providing the input converter specifications remained within the model's region of validity.

In addition to these internal iterations, the entire diode description is continually being used as the surface thermal conditions or other system parameters vary during transients. In order to insure that smooth transitions are made from case to case, previous solutions may be employed. The final converged values of all iterative parameters are stored and subsequently re-used as starting points for the succeeding converter calculation.

Special attention is paid to the initial estimate of the current, as it constitutes the variable for the outside iteration. By using previous values to indicate the direction and slope with which it is currently changing, an initial corrected guess may be made. This allows the thermionic routine to converge usually within two iterations on $J$, rarely more than three. These corrections and initial condition specifications are a11 handled internally within the diode analysis.

The entire thermionic calculation is written so that it can be completely isolated from the remainder of the system description. It is self-contained and requires only the specification of temperatures, load resistance, and other necessary input coniditons. Thus, this portion of the digital program, along with the integration scheme discussed in Chapter II, may be converted into machine language, 
consequently saving extensive compilation time. A complete listing of this transient diode analysis, numerical integrator, and typical system equations for the reactor model discussed in Chapter $V$, are included in the appendix.

Convergence Criteria Used. Due to the necessity of iterative methods for solution, the resulting converged values are known only to within certain prespecified error limits. Most important of these is the accuracy to which the output voltage is determined. Following Wilkins (1968), the diode voltage in this study is calculated to within five millivolts $( \pm 0.005 \mathrm{v})$. Although this limit may be changed, the plasma model used does not warrant any higher degree of accuracy.

The convergence of $V_{O}$ reflects the error of $J$ through Equation (3-23). Since typical values of the load resistance are around five hundredths ohm-centimeter squared $\left(0.05 \Omega-\mathrm{cm}^{2}\right), J$ will be known to approximately one-tenth of an ampere per centimeter squared $\left( \pm 0.1 \mathrm{amp} / \mathrm{cm}^{2}\right)$.

All other iterative parameters are calculated to within one percent of their relative magnitude, or to within an absolute value of 0.01 if their absolute value is less than unity. Again, better accuracy than this is not justified for the plasma analysis utilized.

\section{Use of the Transient Thermionic Analysis}

Specification of Parameters

The required input for the thermionic analysis may be divided into two categories, constant and variable. The constant parameters are those associated with the diode surfaces and interelectrode gas. 
The variable quantities include the gas temperature $\left(T_{R}\right)$, interelectrode spacing $(d)$, load resistance $\left(R_{L}\right)$, and surface temperatures $\left(T_{E}, T_{C}\right)$.

While the gas temperature and spacing are usually held constant during any given run, the possibility of their variance is not precluded. The load is normally specified as either changing incrementally or constant. Whenever any of these parameters are altered, a new diode calculation must be made.

Frequency of Usage

The surface temperatures are determined from the transient thermal equations, and it is their fluctuations that mainly dictate when the thermionic analysis should be used. However, converter calculations at every integration step would lead to prohibitive computational time and, indeed, are not required that frequently. Since most cases run during this study involve transients in which the emitter temperature leads the collector, deviations in the emitter temperature set the thermionic usage criterion. Investigations show that diode computations need only be made whenever the emitter surface temperature changes by five degrees Kelvin $\left(5^{\circ} \mathrm{K}\right)$. The results obtained are of sufficient accuracy to match the responses of calculations made for emitter deviations as low as $1^{\circ} \mathrm{K}$.

Also, as the system transients approach their new equilibria following a perturbation, the situation is reached where the emitter temperature varies less than this prescribed limit. Consequently, the additional criterion is included that diode calculations be made if a 
specified time interval has elapsed since the preceding thermionic computation. Thus, the converter's parameters are adjusted to the new prevailing conditions whenever the emitter changes by $5^{\circ} \mathrm{K}$ or an elapsed time of five seconds has occurred, whichever comes first.

While these limits are flexible, the choice of $5^{\circ} \mathrm{K}$ or 5 seconds has been shown to yield good results. In addition, computational time directly reflects the frequency at which the thermionic analysis is employed. It is therefore advantageous to use the criterion having the widest range while still retaining the desired accuracy.

Initialization

The one remaining problem before analyzing a transient system response is the specification of initial conditions, not only for the integration scheme but also for the thermionic routine. A discussion of an initialization method for the transient thermal equations is delayed until Chapter V.

In order to use any initialization procedure involving the thermionic analysis, the critical parameters within the plasma routine must originally be set at reasonable values. An excellent way to accomplish this, and simultaneously gain a better understanding of converter operation, is to use the steady-state analysis for which SIMCON was originally designed, i.e., the generation of I-V characteristic curves.

Since initial estimates to the emitter and collector temperatures can usually be made, the whole range of possible diode performance at these conditions may be investigated with SIMCON. When 
the initial load resistance is specified, the current and voltage will be set, and appropriate values for the remainder of the critical converter parameters may be selected. A digital program to conduct these steady-state calculations would essentially consist of the transient thermionic analysis listed in the appendix, with the iteration on current deleted.

This steady-state investigation is particularly useful in determining the initial conditions for transient cases involving load changes. In this instance a double set of initial parameters is required to insure the convergence of the thermionic analysis. When the load is altered, a large change is reflected instantaneously by the converter current. The surface temperatures do not change instantaneously, however, so the resulting effect is for the diode to shift its operating point along the initial I-V curve. For the proper analysis of this situation, a new converter calculation must immediately be made. Since the thermionic conditions have changed significantly, it is desirable to start this new computation with the critical iterative parameters near their proper values. From knowledge of the initial surface temperatures, the results of a steadystate $I-V$ curve analysis may be used in specifying these parameters. Once the variables involved are properly initialized, the transient thermionic analysis proceeds automatically as its usage is required during any system run.

Thus, a complete model capable of describing a single thermionic diode experiencing transients has been formulated and its 
versatility discussed. Applications of this model to representations of a thermionic reactor system during start-up transients are discussed in Chapter $V$. Studies to test the validity of the analysis over wide operating ranges as encountered during start-up are described in Chapter IV. Before this is done, however, it is of interest to investigate whether this more complicated converter approach is warranted. Thus, the next section shows the results of comparisons made between the Richardson-Dushman method, discussed in Chapter II, and the current thermionic analysis.

\section{Comparison to Richardson-Dushman Approach}

A simplified thermionic model employing the Richardson-Dushman (R-D) approach was described in Chapter II. It was used there in conjunction with a set of system equations to check the results of a digital computer integration method with those from an analog computer. Here this same system is again studied, but from the standpoint of comparing the $\mathrm{R}-\mathrm{D}$ method with the present diode treatment. To accomplish this, the analysis must first be "calibrated" to steady-state $\mathrm{R}-\mathrm{D}$ results.

Static Comparisons

The R-D model is designed for use with a thermionic reactor system description to investigate perturbations from a normal steadystate condition at full power. To make a proper comparison, the present analysis must be referenced to this initial operating point. This set of reference conditions is listed in Table 3.2. 
As mentioned earlier in this chapter, the thermionic analysis has the capability of being "calibrated" to reproduce any given experimental data. The data to be duplicated here are the I-V curves produced by using the equations $(2-46)$ and $(2-49)$. The resulting $I-V$ curve for the specified reference conditions of Table 3.2 is shown by the solid 1ine $\#_{2}$ in Figure 3.5 .

TABLE 3.2

Reference Operating Conditions

Parameter

Value

Emitter Temperature

Collector Temperature

Diode Spacing

Collector Work Function

Total Emitter Heat Flux

Diode Current

Output Voltage

Load Resistance $2050^{\circ} \mathrm{K}$

$1300^{\circ} \mathrm{K}$

$10 \mathrm{mil}$

2.1 electron volts

58.25 watts $/ \mathrm{cm}^{2}$

$10 \mathrm{amps} / \mathrm{cm}^{2}$

0.6 volts

$0.06 \Omega-\mathrm{cm}^{2}$

The emitter and collector materials of interest here are tungsten and niobium, respectively. Once the required physical constants for these surfaces are obtained, a steady-state I-V curve analysis, such as described in the preceding section, may be conducted. The emitter and collector temperatures are given in Table 3.2 . By adjusting certain surface and plasma parameters (Wilkins and 


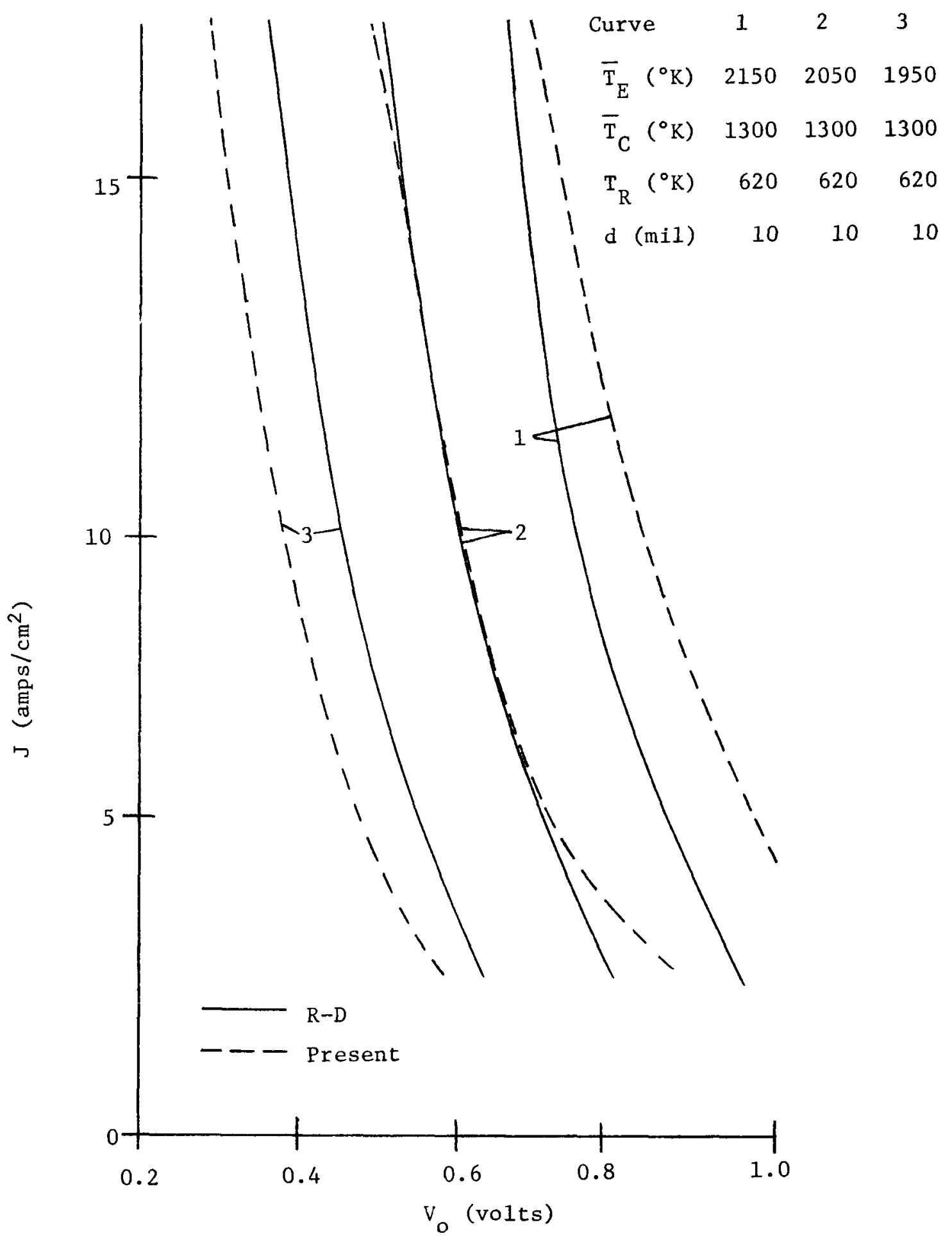

Figure 3.5, Steady-State Comparisons of R-D and Present Thermionic Analysis for Variable $\bar{T}_{E}$ 
McCandless, 1969), the $I-V$ curves produced with the thermionic analysis may be altered somewhat. They may be shifted either right or left, and the slope at higher current values can be varied. These corrections do not change the resulting transport heat values (Equation (3-18)) by any appreciable amount, but rather lead to variations in the output voltage for a specified diode current.

It may be noted that a collector temperature of $1300^{\circ} \mathrm{K}$ is above the normal range as listed in Table 3.1. Thus special attention is paid to the collector parameters, so that its surface analysis converges properly. The appropriate collector values are adjusted so that a field-free work function of 2.1 electron volts is computed for a surface temperature of $1300^{\circ} \mathrm{K}$.

A discrepancy arises between the two thermionic models for the calculation of the radiation and cesium conduction contributions to the emitter heat flux (Equations $(2-52),(2-53)$, and $(3-19),(3-20)$, respectively). The computations by the $R-D$ model are substantially different from those of the present analysis. The cesium conduction term for the R-D model is approximately three times larger than values given by $(3-20)$. The radiation differences are not as gross, but are noticeable. However, since the determination of these quantities does not depend upon thermionic processes, the representations used in the R-D model are also employed here for this particular comparison study.

An important parameter which the R-D approach cannot set or vary is the reservoir temperature. With the final adjustment of this 
as well as the other appropriate input specifications, the resulting I-V curve reproduction as shown by the dashed line $\left.\right|_{2}$ in Figure 3.5 is accomplished. The agreement through the mid-range, where load lines of interest lie, is excellent. The reservoir temperature used to produce this plot is $620^{\circ} \mathrm{K}$. While the comparison to the reference equilibrium I-V curve is good, the agreement worsens as the surface temperatures deviate from these steady-state values.

For $100^{\circ} \mathrm{K}$ deviations in emitter temperature, while holding the collector constant, comparison of corresponding I-V plots between the two methods vary by more than 0.05 volts as shown in Figure 3.5 . It may be noted that the shifts in the I-V curves are in the same direction for both methods, but the effects are significantly greater with the present analysis than the $R-D$ mode 1 .

These differences are even more pronounced when the emitter temperature remains constant and the collector varies. Figure 3.6 shows the resulting comparisons for an emitter temperature of $2050^{\circ} \mathrm{K}$ when the collector temperature changes $50^{\circ} \mathrm{K}$ from its equilibrium value. The R-D curves scarcely move, while those calculated with the present method deviate as much as 0.15 volts.

However, as mentioned above, a $1300^{\circ} \mathrm{K}$ collector temperature is much higher than those of normal operating diodes. Since a major contribution to changes in output voltage during a transient arises from the temperature dependence of the surface work functions, it would be expected that fluctuations in this high collector temperature would be strongly felt. This important effect is not accounted for in the $\mathrm{R}-\mathrm{D}$ model, but is in the present treatment. 


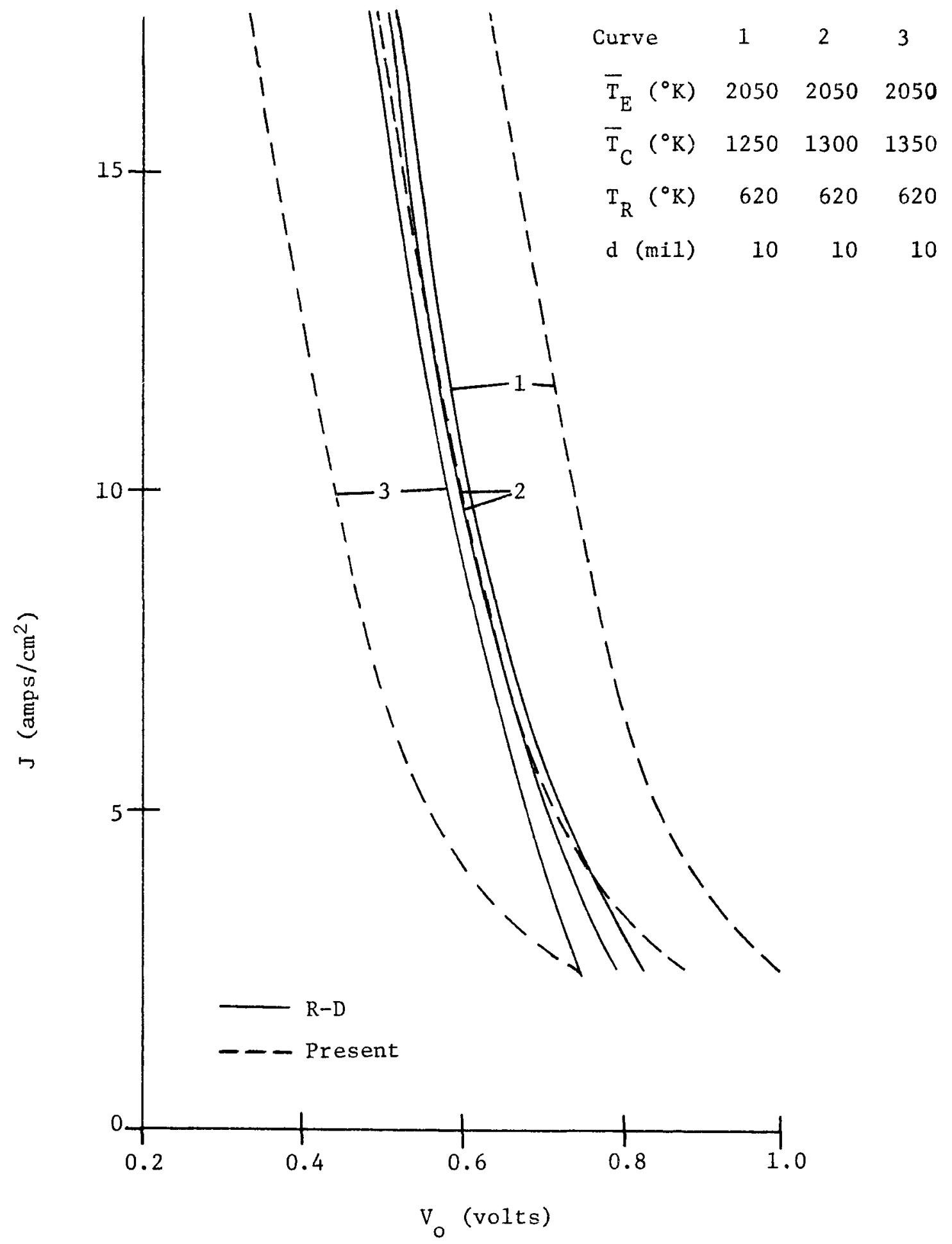

Figure 3.6. Steady-State Comparisons of $R-D$ and Present Thermionic Analysis for Variable $\overrightarrow{\mathrm{T}}_{\mathrm{C}}$ 
These various factors lead to interesting results when transient responses are studied. The comparisons of two such cases are discussed next.

Transient Comparisons

Once the specifications 1 isted in Table 3.2 are satisfied by the thermionic analysis, the remaining system temperatures are automatically equal to those for the transient cases studied in Chapter II. Also, since steady-state calculations are made when matching the reference equilibrium I-V curve, values are available for the critical plasma parameters in the thermionic analysis over the entire range of possible initial converter operating conditions.

The transients studied represent the same cases reported in Chapter II. Since the digital results are shown there to be of better accuracy than those from the analog computer analysis, the digital results are used for the following comparisions.

Positive Step in Power. The system of transient equations used is a duplicate of that shown in Chapter II by Table 2.3. The difference lies in the determination of $q_{E}^{\prime \prime}, J$, and $V_{O}$. $q_{E}$ " is set by Equation (3-18). However, $Q_{r}$ and $Q_{C S}$ are calculated from Equations $(2-52)$ and (2-53), while $Q_{e}$ and $Q_{i}$ come from (3-21) and (3-22). Values of $J$ and $V_{0}$ result from the present thermionic analysis.

With the reactor system operating at equilibrium power, a two-cent step in reactivity is inserted. The resulting transient 
responses of major parameters are shown in Figure 3.7. While power and temperature traces are seen to be quite close, the thermionic properties are substantially different. The somewhat stepwise nature of the thermionic responses of the present method follow because this analysis is not used at every integration point, as discussed earlier.

It is interesting to note that both the diode current and output voltage deviations as predicted by the present treatment are lower than those of the R-D model. A more complete discussion of these results is delayed until the comparisons of the second transient case are shown.

Change in Load. With the system again at its reference equilibrium, a $33 \%$ decrease in resistive load $\left(-0.02 \Omega-\mathrm{cm}^{2}\right)$ is made. Transient results are shown in Figure 3.8. Since no correction is made to maintain equilibrium power, it consequently fluctuates slightly due to thermal reactivity feedback.

The temperature responses are similar qualitatively, but the final emitter values differ by close to $20^{\circ} \mathrm{K}$. Again, the thermionic traces vary significantly, but here the $R-D$ current and output voltage change less than those of the present analysis.

Discussion of Results

As shown by the static comparisons, the present thermionic analysis causes larger deviations in $I-V$ curves than the $R-D$ model for similar temperature fluctuations. Thus, for a given emitter temperature increase, the current and voltage increase more, while for 

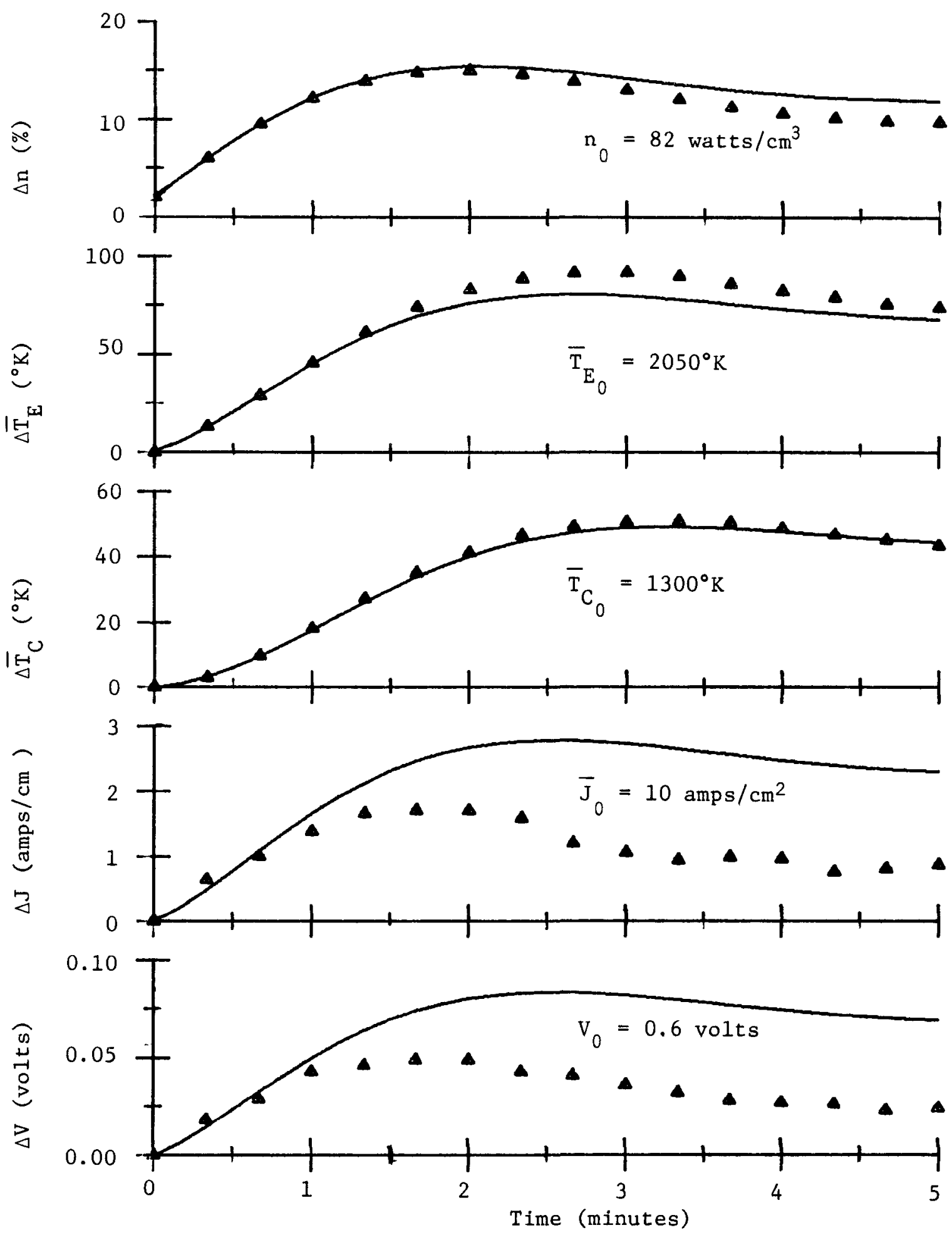

R-D Thermionics

$\Delta \Delta \Delta$ Present Thermionics

Figure 3.7. Comparison of Present Thermionic Analysis to R-D Approach for +2 c Step 

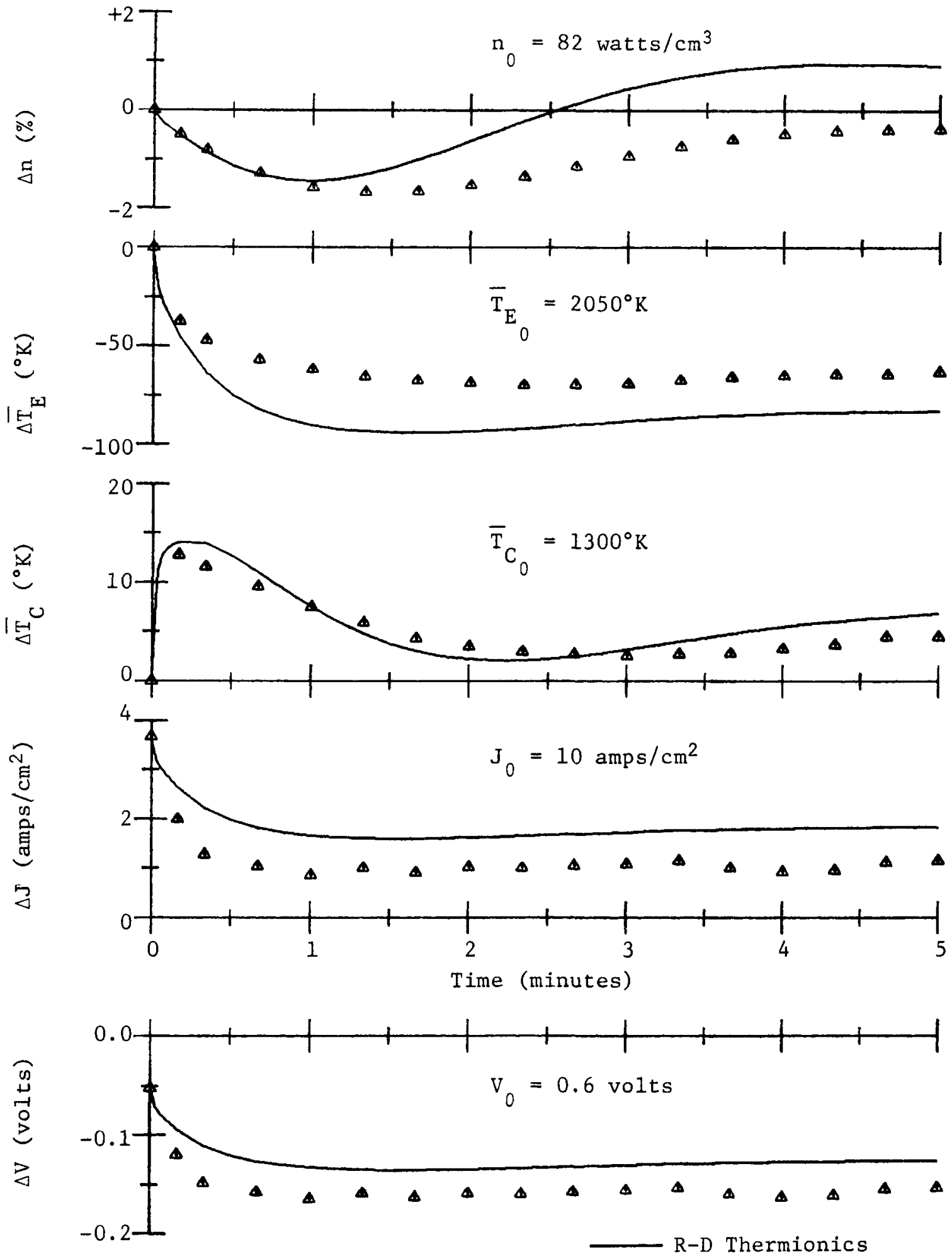

$\Delta \Delta \Delta$ Present Thermionics

Figure 3.8. Comparison of Present Thermionic Analysis to R-D Approach for Change in Load 
a given collector temperature increase, the current and voltage decrease more than the $R-D$ model would predict.

For the case of a positive step in power, both the emitter and collector temperatures increase. Due to the factors just mentioned, the I-V curve deviations tend to cancel each other. The net overall effect is to make the current and voltage changes for the present model less than those for the $R-D$ approach. Also, since less current is being drawn, the emitter temperature for the present system must be slightly higher to account for the heat removal differential.

For a case of load decrease, an immediate large increase occurs in the current. This has the initial effect of cooling the emitter and heating the collector. However, since the total heat removed by the coolant does not change appreciably, the collector eventually returns essentially to its initial value. The emitter, on the other hand, remains at a lower temperature due to the increased effect of electron cooling.

Since the emitter is altered while the collector is relatively unchanged, the current and voltage fluctuations of the present analysis are larger than the R-D model would predict. From Figure 3.8, this initially seems incorrect from two points.

First, the emitter temperature has changed more with the $R-D$ model. However, the magnitude of the differences as shown by the static I-V curve studies overrides this effect.

In the second place, the current change appears to be less with the new approach than with the R-D method. However, with a 
decrease in load, the diode current is perturbed from its equilibrium value. Thus, the current predicted by the present analysis has actually changed more by returning closer to its original value than that of the R-D model.

Also, since electron cooling effects become less as the current lowers toward its original value, the resulting emitter temperature must be hotter. This accounts for the fact that the negative emitter change is less for the present analysis than with the R-D mode1. Therefore, it becomes evident that significant discrepancies exist between the two diode treatments as might well be expected. They are a direct consequence of the inaccuracies inherent in the R-D approach, as indicated by the steady-state comparisons with the present approach. The validity of the present approach has been well established by Wilkins (1968) through comparisons with experimental results of static I-V characteristics. As will be illustrated in the succeeding chapter, the present thermionic analysis also results in good agreement with transient experimental data over wide operating ranges.

The $R-D$ model is very easy to use and does produce a reasonable approximation for the emitter heat $f 1 u x\left(q_{E}^{\prime \prime}\right)$ as witnessed by the relative closeness of the power and temperature comparisons. However, the inadequacies in the calculation of current and voltage values limits the validity of the results to small deviations from equilibrium. Revisions in the approach, notably improvements to account for temperature dependent work functions and collector effects, would be necessary for any large operating condition changes, such as experienced during start-up. In view of the stronger theoretical foundation 
of the present approach, this would hardly be warranted and modification of the $R-D$ model is not recommended. 


\section{CHAPTER IV}

\section{EXPERIMENTAL、STUDIES}

A reactor system model was developed in Chapter II and the required analysis to describe transient converter operations in Chapter III. Before the total reactor system is studied, it is of interest to test the validity of the proposed thermionic analysis method.

As seen in Chapter III, simplified models may be utilized to produce suitable diode results only if the system is restricted to a small region of operation about a given equilibrium point. Coverage of such conditions as start-up, however, entails representation of generalized, not localized transient conditions. Consequently, the analytical description becomes more complex and a number of assumptions and suspected limitations are of necessity involved in the derivation. As a consequence, confidence in the validity of the computational results can only be accomplished from direct verification by experimental data. For these reasons, this chapter deals with the correlation of analytical system response incorporating the thermionic description presented in Chapter III with data obtained from an actual experimental assembly. First, the experimental facility and corresponding set of analytical equations are discussed. Then, some typical comparisons of steady-state and transient results are reported. Also, the 
description of a simplified multiple diode analysis is included. The development of this model is necessary in order to represent a particular transient system response observed experimentally and of considerable interest to the thermionic reactor program.

\section{Experimental Arrangement}

The experimental apparatus is located at the Jet Propulsion Laboratory, Pasadena, California. Initial installation and testing of the facility have been reported (Peelgren, Gronroos, Davis, and Ernst, 1969). Although there are presently four thermionic diodes operating in the enclosed sodium-potassium (NaK) liquid metal coolant loop, these investigations deal with only one. The experimental data analyzed in this chapter were obtained during August 1969.

The experimental assembly is shown schematically in Figure 4.1. The geometry is cylindrical, and the radif indicated are referenced to the centerline.

Power is supplied internally to the diode by an electron bombardment heater. This heat is conducted through a thick rhenium emitter and transported to the collector by the various thermionic processes discussed in Chapter III. The emitter surface area is slightly over $30 \mathrm{~cm}^{2}$. The niobium collector is vacuum-coated with a thin layer of molybdenum on the inner surface and has a 0.010 inch spacing from the emitter.

The outward diode regions include an alumina insulator, niobium sheath, inner Kovar sheath, helium gas-filled gap, and outer 


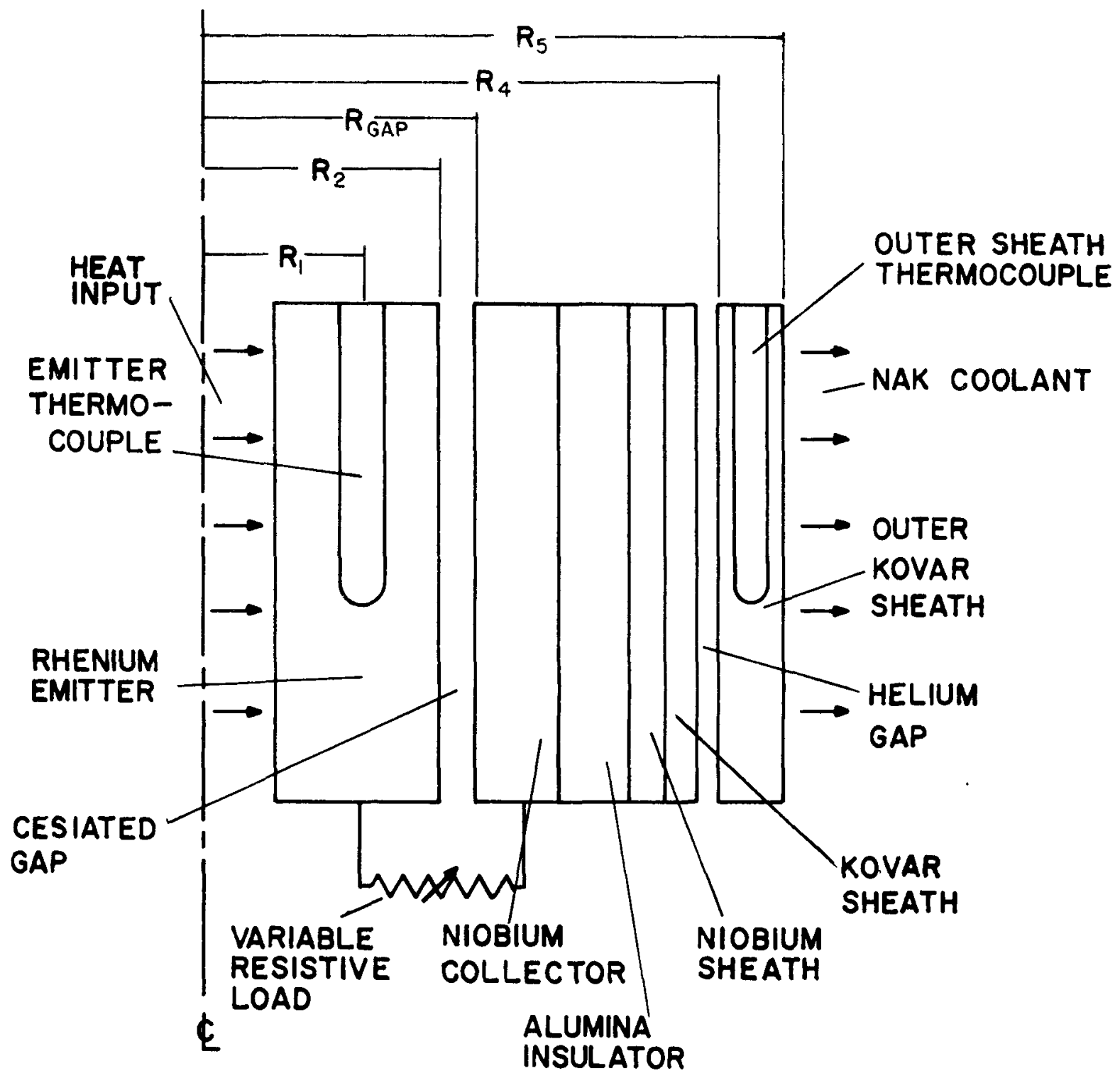

Figure 4.1. Experimental Diode Assembly 
Kovar sheath. The helium spacing permits converter exchanges without opening the liquid metal coolant system.

The exterior surface of the device is cooled by NaK passing in crossflow. The coolant temperature is externally controlled to maintain a constant outer Kovar sheath temperature. Three thermocouples are located at various depths midway through the rhenium emitter, and similarly in the outer sheath. The three rhenium block thermocouples are spaced $120^{\circ}$ apart and placed at approximately 0.35 , 1.05, and 1.75 inch depths into the 2.00 inch long emitter. These are denoted as shallow, middle, and deep thermocouples respectively. This same situation applies to the outer Kovar sheath thermocouples. The electrical circuit for the diode is completed by a mercury resistive load. This load may be changed by pneumatically varying the height of the glass-enclosed mercury column.

The interelectrode gas used is cesium. Its temperature and pressure are controlled separately by an individual cesium reservoir heater.

\section{Analytical Mode1}

For the digital simulation of this system, a one dimensional heat transfer model is developed. The method is analogous to that discussed in Chapter II for the determination of the thermionic reactor equations. A schematic description of the analytical model is shown in Figure 4.2.

The resulting simulation contains five thermal regions. The radii indicated in Figure 4.2 are again referenced to the centerline. 


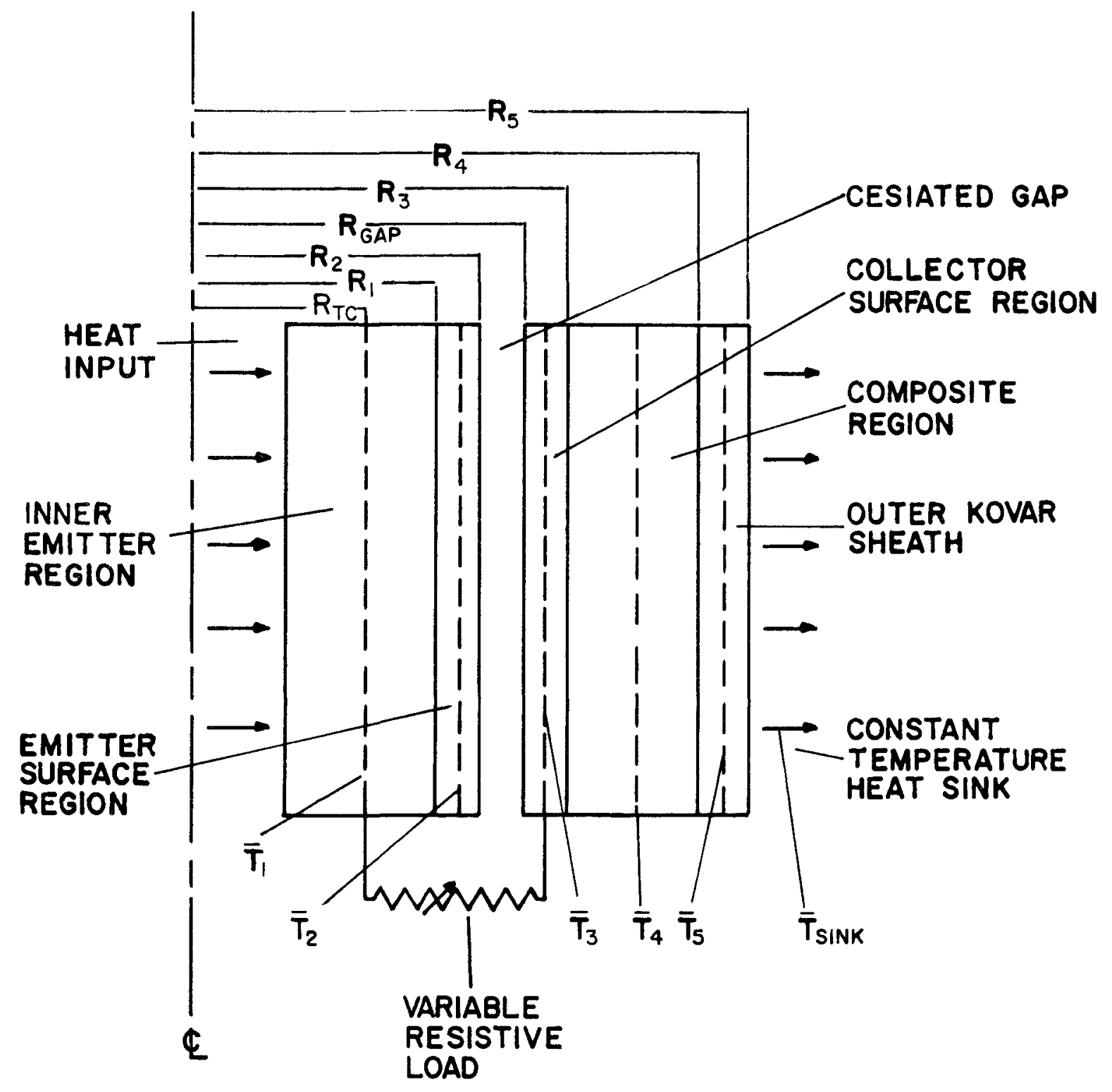

Figure 4.2. Simulated Diode Assembly 
The temperatures shown represent the spatially-averaged values to be determined from the transient analysis.

The thick rhenium emitter is divided into two sections. The emitter block thermocouples are assumed to depict the spatiallyaveraged temperature distribution for the large inner region. A thin ten mil outer portion is retained whose average temperature $\left(\bar{T}_{2}\right)$ is taken to be the emitter surface temperature. This procedure is followed since a difference of up to $50^{\circ} \mathrm{K}$ can occur between the thermocouple and surface temperatures. Thus, this analysis yields a better estimate of the true surface condition for use in the thermionic computations.

Similarly, on the collector side, the inner ten mil thickness of niobium is treated separately in order to represent the average collector surface temperature. The remainder of the niobium, insulator, two sheaths, and helium gap are combined into a single thermal region. The fifth section is the outer Kovar sheath whose average temperature distribution is assumed to be given by the respective thermocouple readings. The coolant is dealt with as a constant temperature heat sink.

Writing expressions in the form of Equation (2-7) for each region, the resulting equation for the inner emitter section becomes

$$
\begin{aligned}
& P V_{1} c_{P_{1}} \frac{d \bar{T}_{1}}{d t}=P-h_{1,2} A_{1}\left(\bar{T}_{1}-\bar{T}_{2}\right) \\
& \bar{T}_{1} \equiv T_{1}\left(R_{T C}\right) ; \bar{T}_{2} \equiv \frac{1}{2}\left[T_{1}\left(P_{1}\right)+T_{2}\left(R_{2}\right)\right] ; A_{1}=2 \pi R_{1} L_{1}
\end{aligned}
$$


where

$$
\begin{aligned}
P & =\text { power supplied to emitter from electron bombardment } \\
\bar{T}_{j} & =\text { spatially-averaged temperature for the } j \text { th } \text { region } \\
h_{1,2} & =\text { effective heat transfer coefficient coupling region } 1 \\
\text { to } 2 & \\
T\left(R_{j}\right) & =\text { spatially-varying temperature evaluated at } R_{j} \\
R_{j} & =\text { values of radii indicated in Figure } 4.2 \\
L_{1} & =\text { length of emitter }
\end{aligned}
$$

The power supplied ( $P$ ) is dictated by the experimental data. However, the total electron bombardment heat input is not transported by thermionic effects because a sizable fraction is lost due to end and structural losses for this particular diode. This point is discussed in more detail later.

$T_{1}$ is taken to represent the linear average of the three emitter block thermocouple readings. While this is the simplest approximation possible, it still yields acceptable results under certain conditions, as will be shown in the subsequent sections.

The emitter surface transient description is written

$$
\begin{aligned}
& P_{2} V_{2} c_{P_{2}} \frac{d \bar{T}_{2}}{d t}=h_{1,2} A_{1}\left(\bar{T}_{1}-\overline{\bar{T}}_{2}\right) \ldots A_{2} q_{E}^{\prime \prime} \\
& A_{2}=2 \pi R_{2} L_{2}
\end{aligned}
$$

where $q_{E}^{\prime \prime}$ is the emitter heat flux as calculated from the thermionic analysis. 
The collector surface temperature response may be expressed

$$
\begin{aligned}
& P_{3} V_{3} c_{2} \frac{d T_{3}}{d t}=A_{2} q^{\prime \prime}-h_{3,4} A_{2}\left(\bar{T}_{3}-\overline{T_{4}}\right) \\
& \bar{T}_{3} \equiv \frac{1}{2}\left[T_{3}\left(R_{j A P}\right)+T_{4}\left(R_{3}\right): T_{4}^{T} \equiv \frac{1}{2} T_{4}\left(Q_{3}\right)+T_{5}\left(R_{4}\right)\right] ; A_{3}=2 \pi R_{3} L_{3}
\end{aligned}
$$

where

$$
\begin{aligned}
& q_{C}^{\prime \prime}=\text { the emitter heat flux less the electrical power } \\
& \text { produced } \\
& h_{3,4}=\text { effective heat transfer coefficient coupling region } 3 \\
& \text { to the composite region } 4
\end{aligned}
$$

The expression for $h_{3,4}$ contains terms for all the materials represented in the single composite portion.

The average transient temperature for this collapsed region is of the form

$$
\begin{aligned}
& P_{4} V_{4} c_{p_{4}} \frac{d \overline{T_{4}}}{d t}=h_{34} A_{3}\left(\overline{T_{3}}-\overline{T_{4}}\right)-h_{4,5} A_{4}\left(\bar{T}-\overline{T_{5}}\right) \\
& \bar{T} \equiv \frac{1}{2}\left[T_{5}\left(R_{4}\right)+T_{5}\left(R_{5}\right) ; A_{4}=2 \pi R_{4} L_{4}\right.
\end{aligned}
$$

where $h_{4,5}$ is the effective heat transfer coefficient coupling region 4 to 5. The term, $P_{4} V_{4} C_{P_{4}}$, consists of a summation of these individual products for the materials contained in region 4.

The equation for the final section, whose average temperature typifies the 1inearly-averaged value of the three outer Kovar sheath thermocouples, may be described 
${ }_{5} V_{5} c_{F_{5}} \frac{d \bar{T}_{5}}{d t}=h_{4,5} \hat{A}_{5}\left(\bar{T}_{4}-\bar{T}_{,}\right)-r_{5, N A \mu} s_{5}\left(T_{b}-\bar{T}_{S, 1 k}\right)$

$$
L_{5}=2 \pi F_{5} L_{5}
$$

where $h_{5 \mathrm{NaK}}$ is the effective heat transfer coefficient coupling

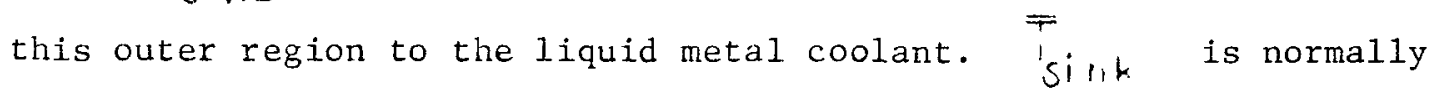
maintained at $800^{\circ} \mathrm{K}$.

All the pertinent parameters required for the determination of the various equations are listed in Table 4.1. The resulting set of equations with the respective time constants is shown in Table 4.2.

\section{Comparison Studies}

An appropriate analytical model is now fully described. The actual comparison studies may be divided into two categories; steadystate and transient correlations. As seen in Chapter III, the closeness of the transiert. matching is a direct consequence of the accuracy to which the steady-state I-V curves are determined.

Steady State Analysis

Whenever an experimental steady-state converter operating condition was to be examined, the diode was initially brought to the desired temperature and output current values by varying the input power and the resistive load. Data measurements obtained for the point under analysis included diode current, diode voltage, load resistance, input power, cesium temperature, three emitter block thermocouple readings, and three outer Kovar sheath thermocouple readings. 
TABLE 4.1

Parameters for Experimental Studies

\begin{tabular}{|c|c|c|c|c|c|}
\hline & $\rho_{j}$ & $\mathrm{C}_{\mathrm{P}_{j}}$ & $\mathrm{~K}_{j}$ & $\mathrm{R}_{j}$ & $\mathrm{~L}_{j}$ \\
\cline { 2 - 6 } & $\mathrm{gm} / \mathrm{cm}^{3}$ & $\mathrm{w}-\mathrm{s} / \mathrm{gm}^{\circ} \mathrm{K}$ & $\mathrm{w} / \mathrm{cm}^{\circ} \mathrm{K}$ & $\mathrm{cm}$ & $\mathrm{cm}$ \\
\hline $\mathrm{Re}$ & 20.3 & 0.17 & 0.47 & 0.95 & 5.08 \\
$\mathrm{Nb}$ & 8.42 & 0.27 & 0.56 & 1.13 & 5.08 \\
$\mathrm{Al}_{2} \mathrm{O}_{3}$ & 3.2 & 1.24 & 0.061 & 1.28 & 5.08 \\
$\mathrm{Nb}$ & 8.44 & 0.27 & 0.54 & 1.31 & 5.08 \\
Kovar & 8.0 & 0.51 & 0.11 & 1.359 & 5.08 \\
He (gap) & - & - & 0.003 & 1.361 & 5.08 \\
Kovar & 8.0 & 0.51 & 0.11 & 1.61 & 5.08 \\
\hline
\end{tabular}


TABLE 4.2 .

Dynamic Equations for Experimental Studies

$$
\begin{aligned}
& \frac{d \bar{T}_{1}}{d t}=0.0242 T^{\prime}-0.93\left(\bar{T}_{1}-\bar{T}_{2}\right) \\
& \frac{d \bar{T}_{2}}{d t}=14.28\left(\bar{T}_{1}-\bar{T}_{2}\right)-11.74 q_{E}^{\prime \prime} \\
& \frac{{ }^{T_{3}}}{d t}=16.547_{c}{ }^{\prime \prime}-10.35\left(\bar{T}_{3}-\overline{T_{4}}\right) \\
& \frac{d T_{4}}{d t}=0.43\left(\bar{T}_{3}-\bar{T}_{4}\right)-0.207\left(\bar{T}_{4}-\bar{T}_{5}\right) \\
& \frac{d T_{5}}{d t}=0.268\left(T_{4}-F_{5}\right)-0.034\left(T_{5}-T_{\operatorname{sink}}\right)
\end{aligned}
$$


The experimental capability existed to superimpose a sixty cycle voltage sweep onto the converter output terminals. When this was done, and the resulting oscilloscope trace photographed, I-V curves like that shown in Figure 3.4 could be produced.

By varying the diode conditions, such plots could be tabulated over wide ranges of converter operation. These steady-state data were subsequently used to "calibrate" the thermionic analysis.

The experimental voltage values of the resulting traces, however, do not represent the true diode output voltage since the voltage taps are located external to the actual converter structure. Thus, these internal lead losses must be accounted for.

A temperature dependent emitter-collector structural

resistance is calculated using tabulated values for the materials involved (Kohl, 1967). The resultant resistance, calculated for the specific experimental assembly, ranges from 500 to 600 micro-ohms $(\mu-\Omega)$. Consequently, experimental comparisons to the analytical voltage computations are expressed

$$
V_{0}=V_{D}+J \cdot R_{E, C}
$$

where

$$
\begin{aligned}
& V_{O}=\text { experimental voltage for comparison to that calculated } \\
& \text { from thermionic analysis } \\
& V_{D}=\text { experimentally measured diode voltage } \\
& J=\text { experimental converter current } \\
& R_{E C}=\text { emitter-collector lead resistance }
\end{aligned}
$$


The emitter surface temperature is assumed uniform for diode calculations. However, the three experimental thermocouple responses are in fact quite spread out due to nonuniform electron bombardment heating. These temperature differences are frequently as much as $150-200^{\circ} \mathrm{K}$ per axial inch over the two inch long emitter. Still, for the steady-state studies, the linear average of these three readings is taken to represent the bulk emitter temperature ( $T_{1}$ in Figure 4.2). Similarly, the surface temperature $\left(T_{2}\right.$ in Figure 4.2) varies in this manner, but is considered identical at each point. Further treatment of the emitter temperature analysis is discussed in the next section. The collector surface temperature $\left(\bar{T}_{3}\right)$ is much less affected by this uneven heating and is almost uniform.

The steady-state studies can now proceed once the required input parameters for cesium gas, rhenium emitter, and molybdenum collector are set for the converter physics analysis. Even though the bulk collector material is niobium, it exhibits the thermionic properties of molybdenum since a thin layer of this substance has been vacuumcoated onto the inner surface.

With the specification of surface and cesium temperatures, known diode spacing, and calculated lead loss resistance, the steadystate "calibrations" may be accomplished. By varying certain parameters, as mentioned in Chapter III, the thermionic analysis results can be adjusted to enable better matching to experimental $\mathrm{I}-\mathrm{V}$ data. Typical comparisons obtained in this study are shown in Figure 4.3. The actual analytically calculated points are represented 


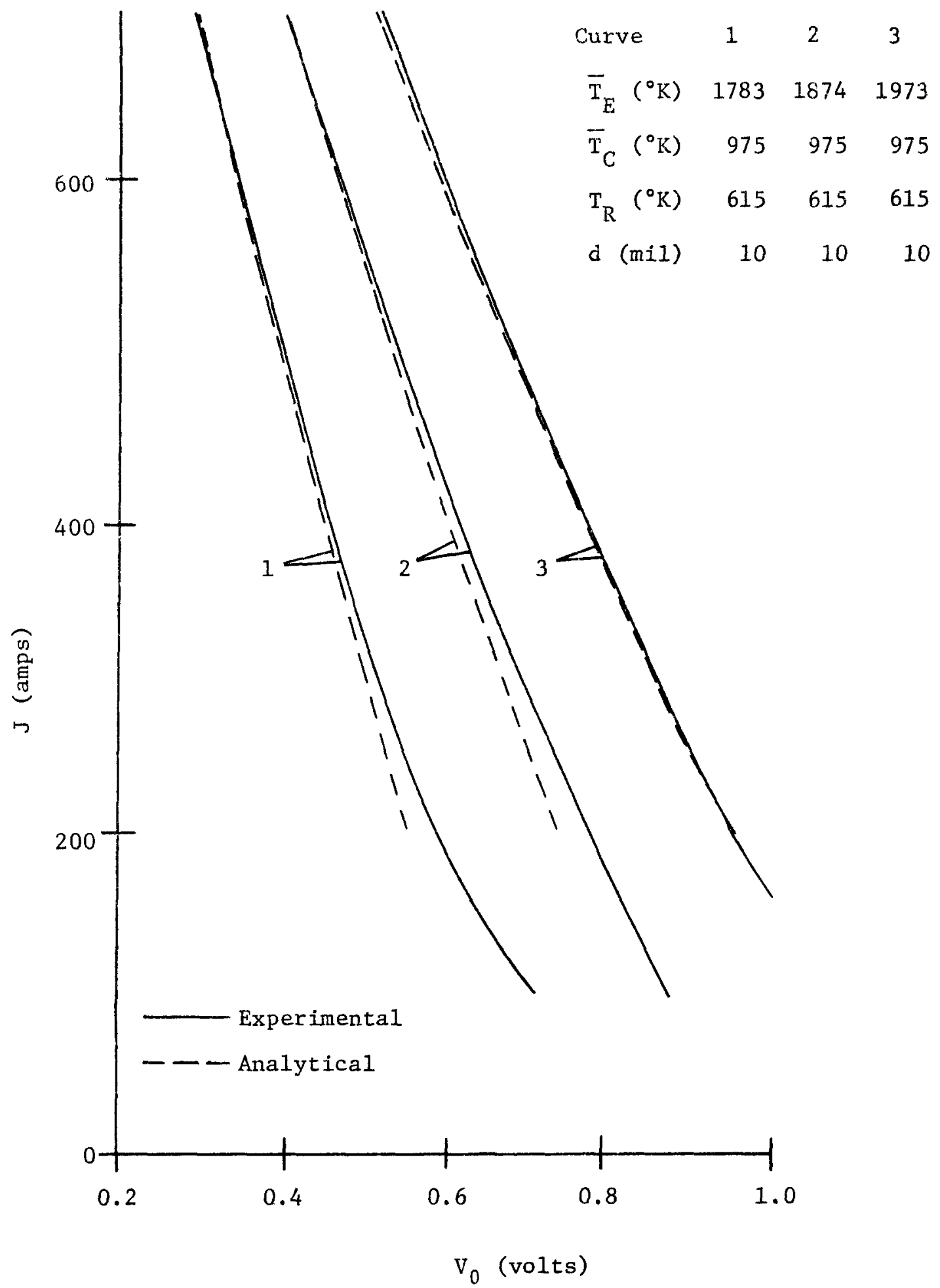

Figure 4.3. Steady-State Comparisons of Experimental and Analytical I-V Curves 
while the experimental voltage values are corrected by Equation (4-6) to yield the true diode output voltage.

The correlations are excellent at higher currents, falling off slightly at lower levels. However, load lines of interest all run through the mid-range. Thus, with the thermionic analysis properly matched to steady-state experimental response, applications to transient cases can now be investigated.

Transient Analysis

The procedure for recording experimental data during any transient case involved three steps. First, the initial conditions of all pertinent parameters were obtained. Next, the perturbation was introduced and the transient responses of the important variables were monitored to produce real-time traces. These plotted quantities included the three bulk emitter thermocouple readings, middle kovar sheath thermocouple, total diode current, and output voltage as measured across the external taps.

Finally, the final equilibrium operating conditions were measured when the system reached its new steady state. The results of a number of such experimental cases were tabulated for analytical comparisons.

Three Parallel Diode Model. Preliminary studies indicated that the transient analytical diode current and load voltage results produced good matches to experimental values. However, the average bulk emitter temperature correlation was inferior, particularly for cases involving changes in the resistive load. 
Since the representation of the emitter surface by a single temperature was known to be in substantial error as indicated by the three emitter block thermocouples, this poor correlation was not too surprising. A first attempt at improvement consisted of formulating a diode representation in which the emitter region was divided into three equal volume sections. Each of these regions had an individual bulk emitter temperature depicted by the respective thermocouple reading.

Analytically, the representation is identical to the case of three diodes operating in parallel (see Fig. 4.4). The diode current from each region adds to the total current, but the output voltage for each section must be equal, since the converters are coupled in parallel. Thus, the thermionic iterative condition to be satisfied (comparable to Eq. (3-23) for one diode) becomes

$$
\begin{aligned}
& V_{O_{j}}=\left(J_{D}+J_{M}+J_{S}\right) R_{T} \quad(j=0, M, s) \\
& R_{T}=K_{E, C}+R_{L}
\end{aligned}
$$

where

$$
\begin{aligned}
& \text { subscript } D=\text { deep; } M=\text { middle; } S=\text { shallow } \\
& V_{O_{j}}=\text { analytically calculated output voltage for } j^{\text {th }} \text { region } \\
& J_{j}=\text { analytically calculated output current for } j^{\text {th }} \text { region } \\
& R_{L}=\text { load resistance }
\end{aligned}
$$




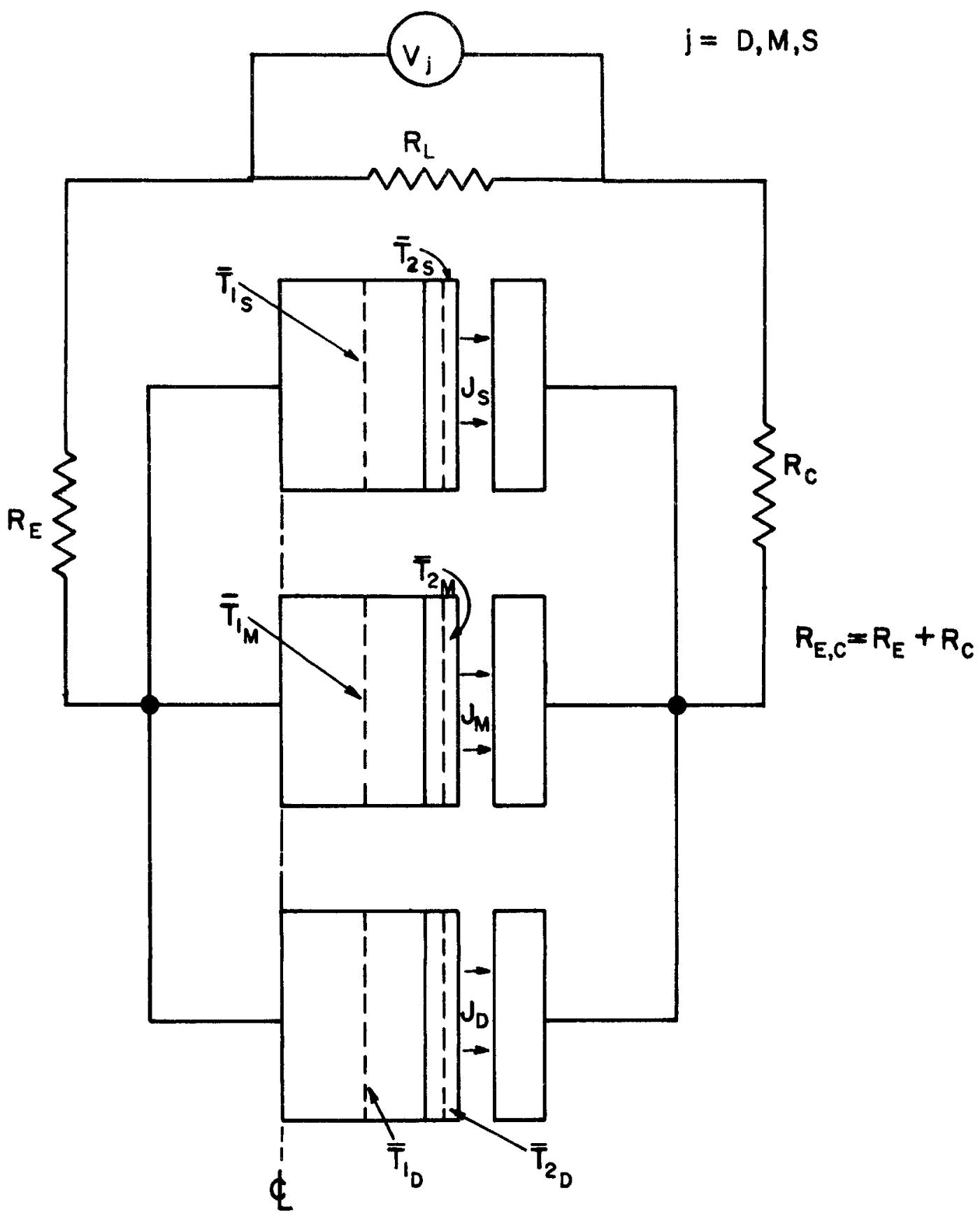

Figure 4.4. Three Paralle1-Connected Diode Model 
To simplify the required analysis for this three diode model, the collector surface temperature was assumed Invariant at all times. Since the coolant was controlled to maintain a constant outer sheath temperature, preliminary transient analytical studies showed that the

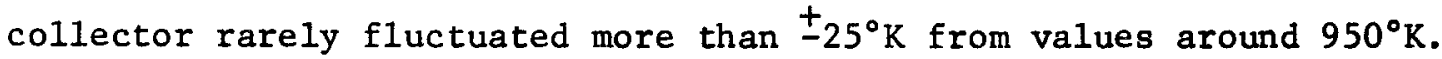
Further steady-state calculations showed that for a given emitter surface temperature and diode current, the output voltage changed about $\pm_{0.02}$ volts by varying collector temperatures in this range. Also, the resulting emitter heat flux $\left(q_{E}^{\prime \prime}\right)$ was affected less than 0.5 watts $/ \mathrm{cm}^{2}$. Thus, a fixed collector surface temperature of $950^{\circ} \mathrm{K}$ was felt to be a reasonable approximation for this diode under controlled coolant operation.

If the transient cases to which the multiple converter model is compared are restricted to those involving constant reservolr temperature, the complex thermionic analysis may be replaced by a doubly dimensioned library routine. For constant collector and cesium temperatures, and given values of diode spacing and load resistance, the remaining variables required to specify a thermionic operating point are the emitter temperature and output current. Once these are set, the diode voltage and emitter heat flux may be determined.

Thus an extensive but simple cataloging procedure was incorporated replacing the detalled thermionic analysis. Steady-state I-V curves were calculated holding the collector temperature at $950^{\circ} \mathrm{K}$ and the cesium reservoir at $615^{\circ} \mathrm{K}$. For each plot, the current was varied from 1.0 to 25.0 amps $/ \mathrm{cm}^{2}$ by increments of $1.0 \mathrm{amp} / \mathrm{cm}^{2}$. Also, I-V curves were determined for emitter temperatures from $1700^{\circ} \mathrm{K}$ 
to $2300^{\circ} \mathrm{K}$ by increments of $10^{\circ} \mathrm{K}$. The resulting computations of voltage and emitter heat flux were stored in doubly dimensioned arrays. Linear interpolations were employed to calculate these variables for given current and emitter temperature values.

The constant collector approximation also reduces the transient thermal analysis. Equations (4-3) to (4-5) for each diode region may be neglected. Thus, the variables to be specified for each section are a bulk emitter temperature $\left(\bar{T}_{1 j} ; J=0, M, s\right)$ and an emitter surface temperature $\left(\bar{T}_{2 j} ; J=C, M, S\right)$. The appropriate describing equations may be written

$$
\begin{aligned}
& P_{1} \frac{V_{1}}{3} c_{p_{1}} \frac{d \bar{T}}{d t} \bar{T}_{1}=f_{j}-h_{1,2} \frac{A_{1}}{3}\left(\bar{T}_{1 j}-\bar{T}_{2 j}\right) \\
& P_{2} \frac{V_{2}}{3} c_{p_{2}} \frac{d \bar{T}_{2 j}}{d t}=h_{1,2} \frac{A_{1}}{3}\left(\bar{T}_{1 j}-\bar{T}_{2 j}\right)-\frac{A_{2}}{3} q_{E j}^{\prime \prime}
\end{aligned}
$$

where $f_{j}$ is the fraction of input power supplied to the $j{ }^{\text {th }}$ region. These fractions $\left(f_{j}\right)$ are not equal due to the nonuniform electron bombardment heating. Indeed, an important result of this multiple diode analysis shows that the amount of heat conducted through each region actually varies during a transient. This fact is clearly evident when the initial and final equilibrium experimental values for any transient case were examined with the three diode model. To account properly for the resultant experimental emitter temperature distributions, it was found that the fraction of input 
heat transported from each region at the end of a given transient run was not necessarily the same as the initial fraction. This was especially true for cases involving changes in the resistive load during which the diode current varied significantly.

It should be noted here that in all experimental tests conducted, the middle emitter temperature always remained hotter than either the deep or shallow. Correspondingly, the current from this region was higher.

As mentioned previously, the total power supplied by the electron bombardment heater is not all transported across the gap by thermionic processes. A certain portion is dissipated from the extremities of the diode by radiation and other effects. It is assumed that this fraction must be a function of the prevailing diode current as we11 as temperature.

To illustrate, consider a transient case in which the resistive load is suddenly decreased. This drop in resistance is immediately countered by an increase in diode current. This in turn cools the emitter, particularly the middle section, since it is producing more current. Presumably this has the overall effect of drawing more power into the diode which otherwise would have been lost off the ends.

The validity of this presumed sequence was borne out by a systematic check of measured experimental data. Analysis showed that the total fractional heat removed by thermionic processes varied consistent1y from about $65 \%$ at 250 amperes to $80 \%$ at 425 amperes for the transient cases recorded. 
With this additional insight, the comparison studies were rerun. Now, however, the experimental data at both initial and final conditions were used to determine the respective fractions of thermal power to be accounted for by thermionic effects. The results of three such cases for both the single and triple diode analyses are discussed next.

Change in Power. With the experimental converter operating at equilibrium, a $35 \%$ change in total input power was ramped in over a thirty-second period. This was accomplished manually by increasing the electron bombardment heater's filament voltage. The load resistance was held constant during the transient. Analtyical comparisons to experimental response are shown in Figures 4.5 and 4.6 .

Figure 4.5 illustrates the results of employing the three diode model. The temperature-time traces compare the actual experimental bulk emitter temperature deviations to the analytically calculated responses of $\bar{T}_{\jmath}(j=0, M, S)$, as determined from Equations (4-9). The computed current curve represents the sum of $J_{j}(J=L, M, S)$ resulting from the thermionic routine. The analytical voltage plot depicts the variation of $V_{M}$ as set by Equation (4-7), but corrected for lead resistive losses to produce the proper values for matching to experimental data. The initial conditions for the respective temperature traces are indicated.

For comparison, the results of employing the single diode analysis are shown in Figure 4.6. The temperature graph represents the comparison of the linearly-averaged change in the emitter 

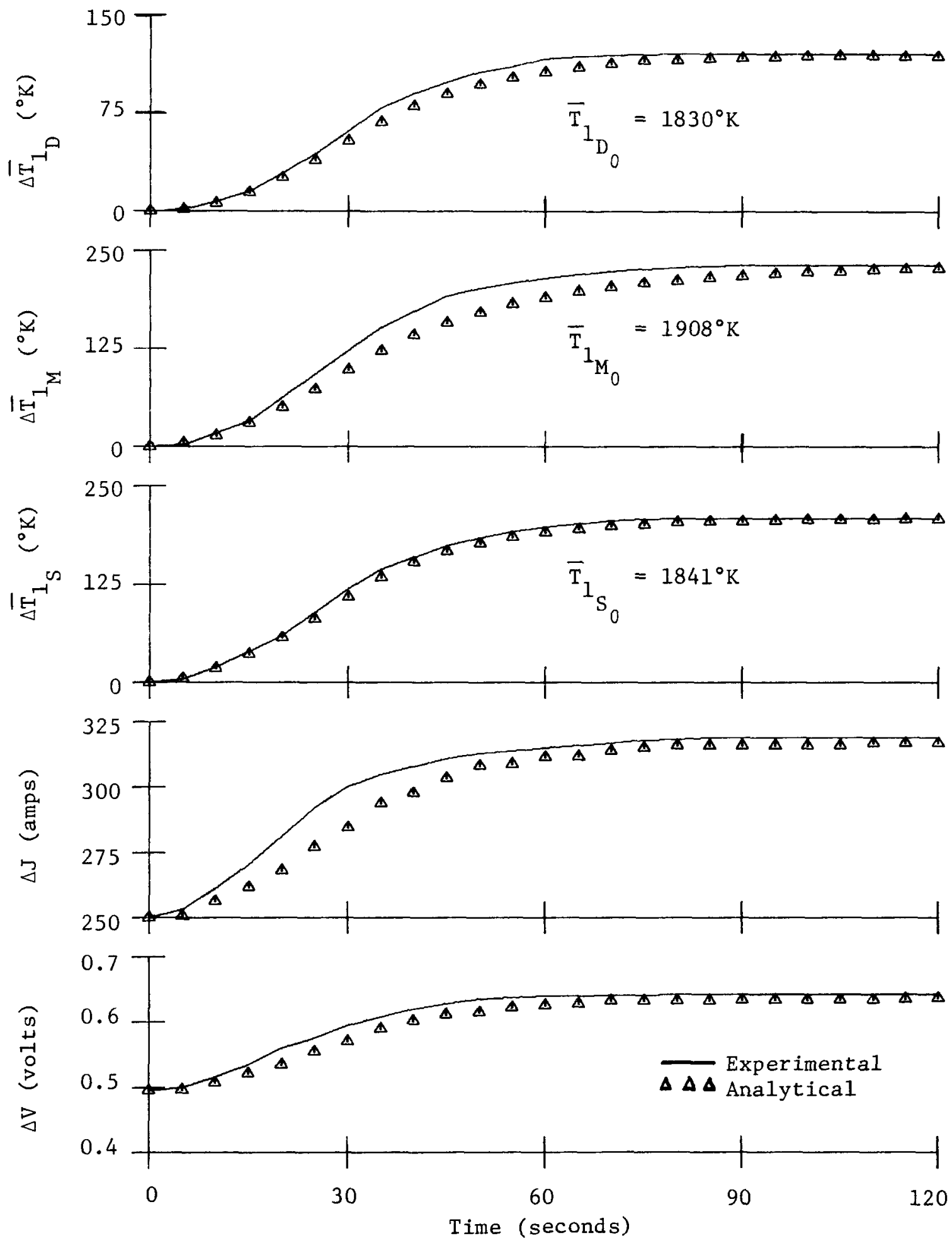

Figure 4.5. Comparison of Three Parallel Diode Model and Experimental System Responses for + Power Ramp 

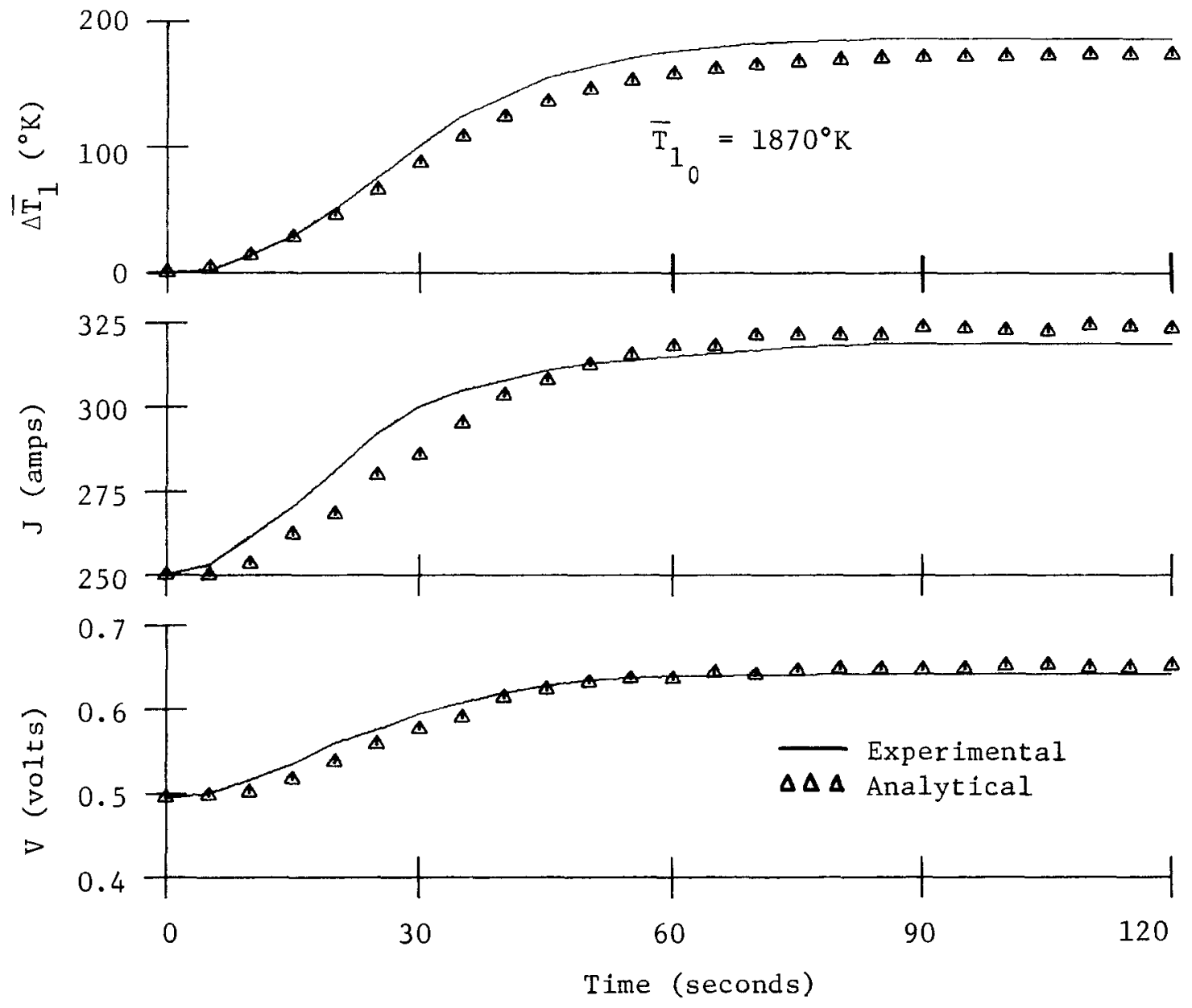

Figure 4.6. Comparison of Single Average Diode Model and Experimenta1 System Responses for + Power Ramp 
thermocouples to that of $\bar{T}_{1}$, as determined from Equation (4-1). For this model, the plotted diode current and lead-loss corrected voltage values are calculated using the transient thermionic analysis described in Chapter III, rather than the cataloging routine employed with the three diode representation.

As can be seen, the correlation with experimental data is very good for both analytical models. It may be noted that this power transient produces a substantial change of almost $200^{\circ} \mathrm{K}$ in the average emitter temperature. A further discussion of results follows the presentation of two more typical comparison cases.

Change in Load. With the system operating at steady state under constant total input power, the pneumatically controlled mercury load was rapidly decreased by $50 \%$. The resulting transients are depicted in Figures 4.7 and 4.8. Although the resistance perturbation actually occurred as a fast ramp, it was programmed analytically as a step.

As expected, the increase in electron cooling following the large positive change in diode current causes the emitter temperature to decrease. The close comparison between the temperature responses is a consequence of the preliminary analysis with the three diode model utilizing the initial and final operating points. For this case involving a significant increase in output current,(50\%) it is found that the fraction of total power actually conducted through the diode rises from $69 \%$ to $79 \%$ during the ensuing transient. As with the previous case, both analytical representations provide excellent simulations of the transient response. 

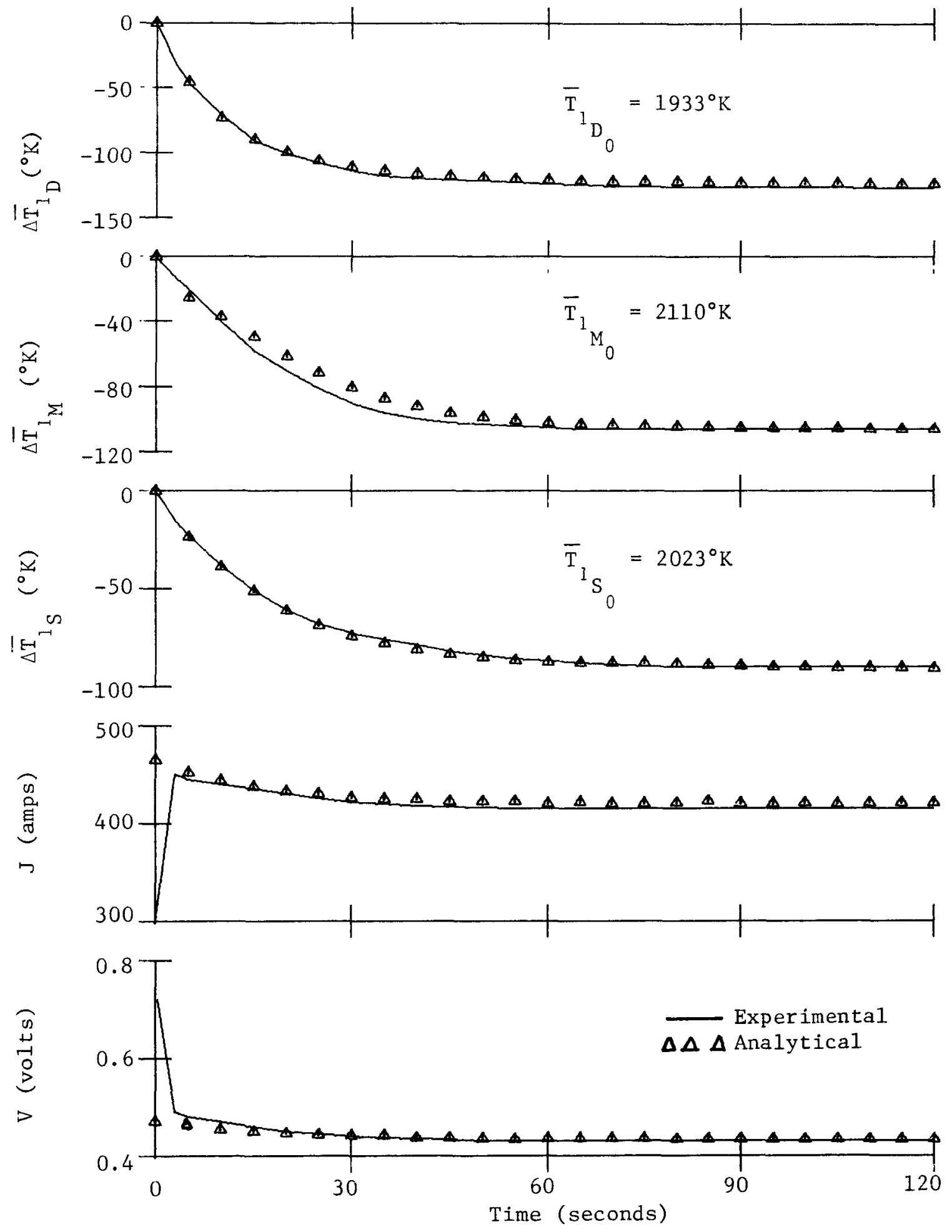

Figure 4.7. Comparison of Three Parallel Diode Model and Experimental System Responses for Decrease in Load 

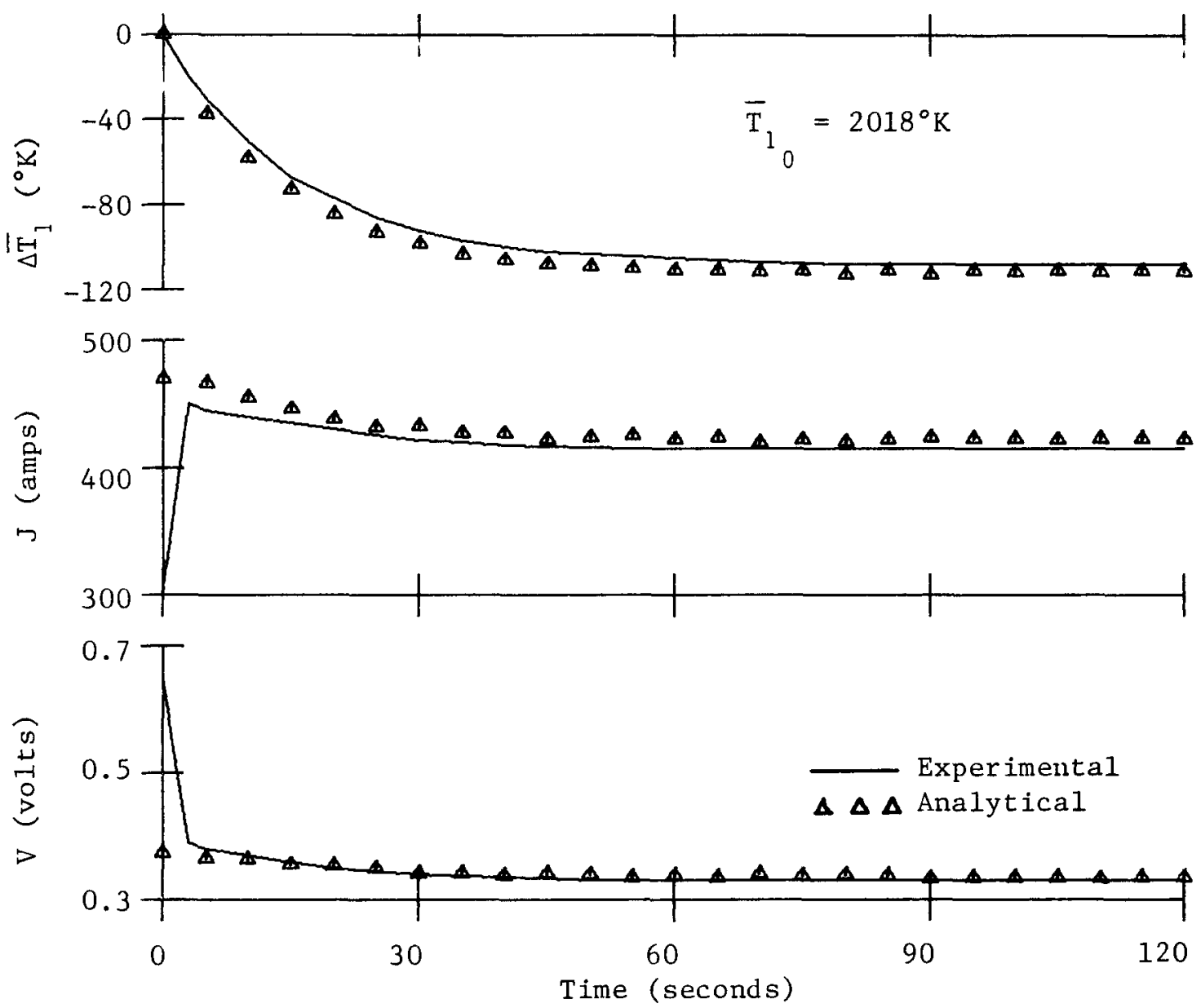

Figure 4.8. Comparison of Single Average Diode Model and Experimental System Responses for Decrease in Load 
Thermionic Burnout. Before the results of this particular case are shown, a brief discussion of thermionic burnout is warranted. Since this potentially dangerous situation may arise in any cesiated diode, a converter model should properly account for its occurrence Whenever mentioned previously in this study, it has been tacitly assumed that diode current and, consequently, emitter heat flux are monotonically increasing functions with rising emitter temperature. This condition is valid over most of the converter's operating range of interest. However, an upper limit is reached where additional heat input causes the actual output current to drop. Because of the loss of electron cooling, the emitter temperature can increase significantly. This phenomenon, called thermionic burnout (Schock, 1968), is represented for a typical case in Figure 4.9. For a diode operating under constant load and cesium temperature conditions, the direct effect of raising the emitter temperature is to increase the current density. However, the cesium coverage is continually decreasing with increasing emitter temperature, thereby raising the emitter work function toward its bare surface value, and indirectly lowering the current density basically through equation 3-13. Above some critical emitter temperature this indirect effect predominates with the end result of decreasing the total heat transported.

In order to counter this situation of diminishing electron cooling, the emitter temperature must raise drastically to balance the required heat removal with increased thermal radiation. This unstable 


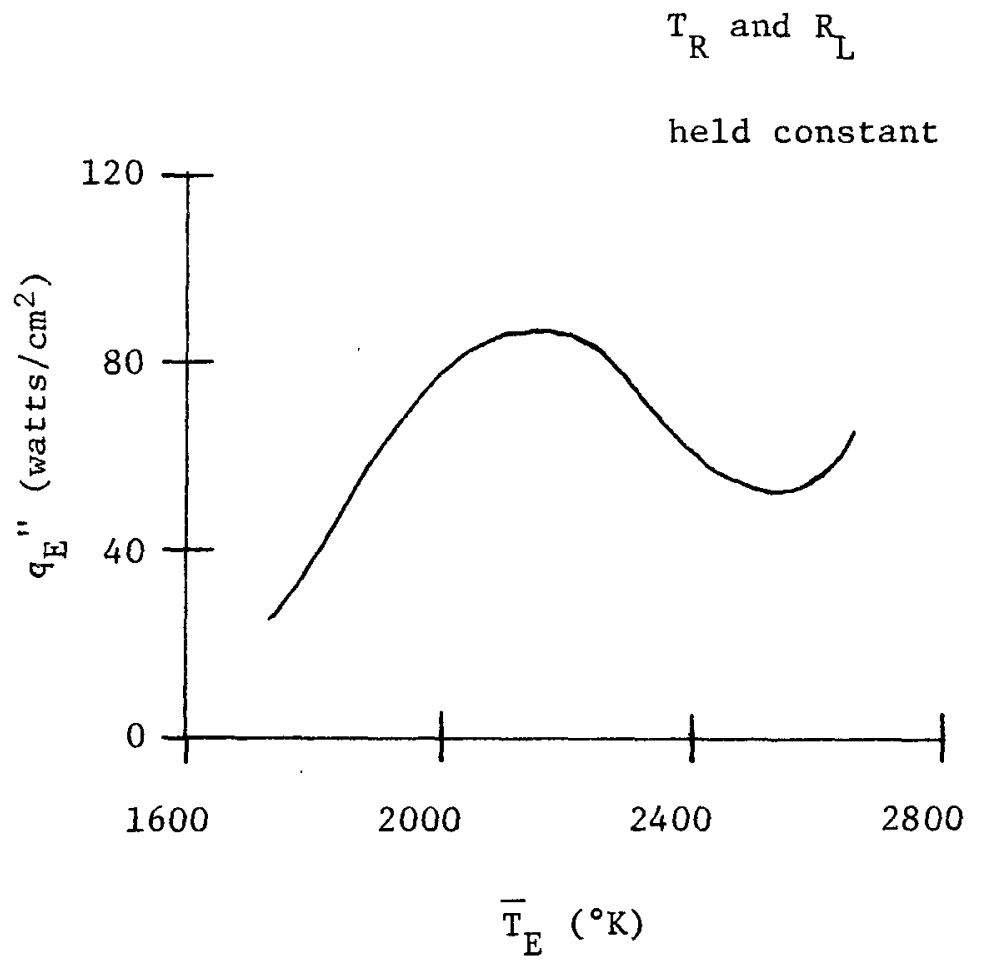

Figure 4.9. Effect of Heat Input on Emitter Temperature at Constant Reservoir Temperature 
response is very undesirable as it can lead to material meltdown, analogous to the burnout condition encountered in boiling heat transfer.

The last case studied here deals with a diode transient during which thermionic burnout inadvertently began to occur. The initial perturbation was a duplicate of that previously discussed for the power change run. However, the $35 \%$ increase in power was inserted as a step instead of a ramp, as previously.

The resulting rapid rise in emitter temperature eventually caused the automatic controller for the experimental power supply to become unstable, and at twenty-two seconds into the transient this caused an additional $15 \%$ in power to be inserted over a twenty-second period. Before this situation was finally noticed and the operation safely terminated, the diode, led by the hotter middle emitter region, passed into the initial stages of a burnout excursion.

The comparison studies are illustrated in Figures 4.10 and 4.11. It is of major importance to note that while the triple diode model substantiates the ensuing trend toward thermionic burnout, the single converter system does not. The actual burnout is evidenced analytically in Figure 4.10 by the rapid increase in middle emitter temperature while the total diode current is simultaneously diminishing.

The results of the single diode response (Fig. 4.11) indicate that a non-burnout equilibrium is achieved. Further tests were conducted with this model. It was found that an additional increase 

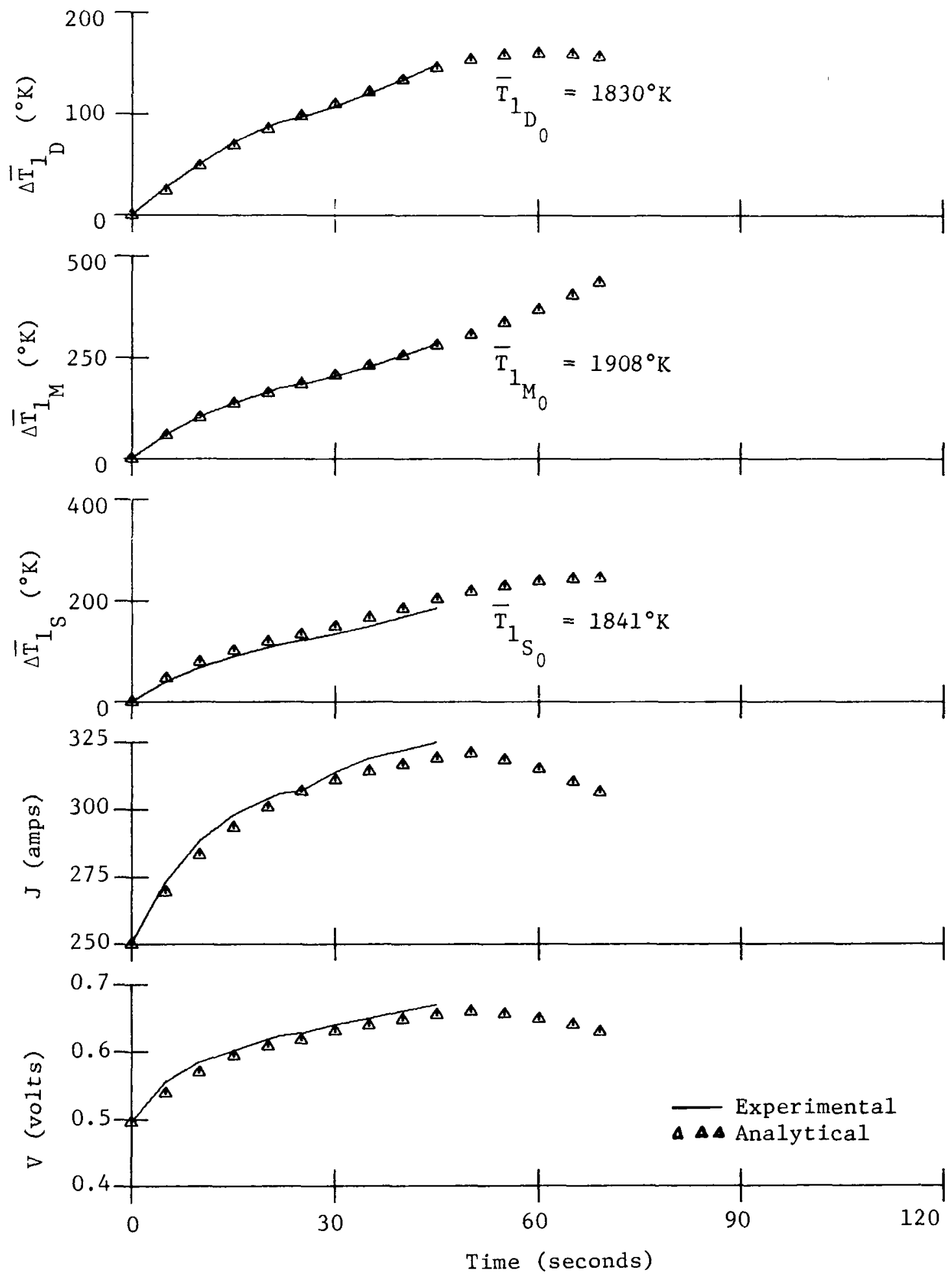

Figure 4.10. Comparison of Three Parallel Diode Model and Experimental System Responses for Thermionic Burnout 

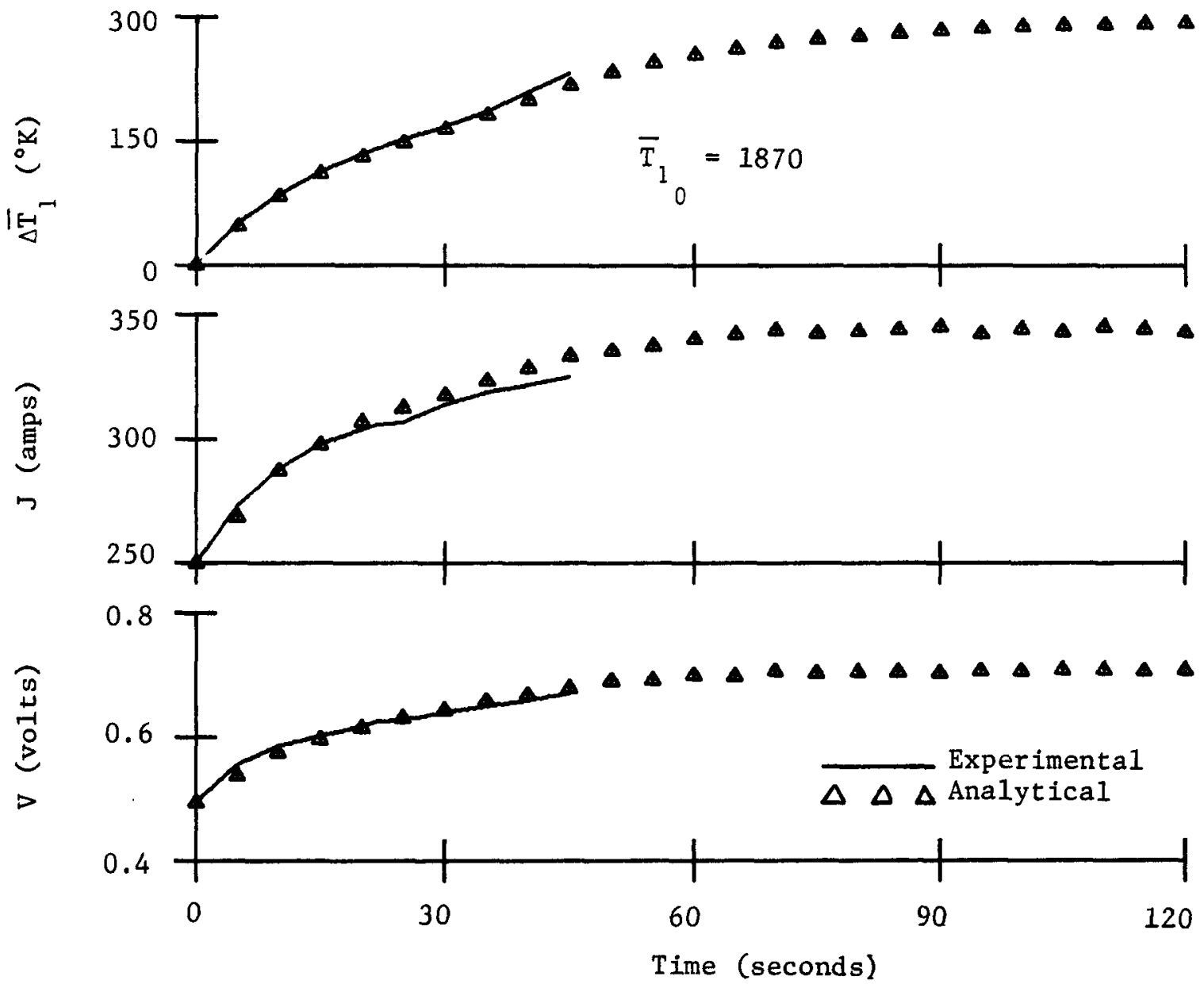

Figure 4.11. Comparison of Single Average Diode Model and Experimental System Responses for Thermionic Burnout 
in input power of more than $5 \%$ (around 150 watts) was required to drive the transient thermionic analysis into the burnout regime. The fact that the discrepancies shown in Figures 4.10 and 4.11 exist is an interesting and important consequence. Thus, in some instances, a constant surface temperature approximation to describe an actual thermionic converter with nonuniform emitter temperature distribution can be inadequate.

Discussion of Results

The conclusions to be drawn from the above comparison cases are twofold. First, the thermal response of a thermionic diode may be reasonably approximated by a model incorporating spatiallyaveraged temperatures and one dimensional heat flow. The values of the resulting time constants for transient analysis produce good qualitative and quantitative estimates to an actual system operation. This fact is evidenced not only by the clear correlation of the final values for the temperature traces but also by the close agreement of the rate of change during transients.

Second, these studies demonstrate convincingly that the transient thermionic analysis developed in Chapter III can indeed accurately predict experimental diode fata. The comparisons are excellent over wide ranges of possible system response and are equally valid for any given type of perturbation. The assumption of uniform surface temperature is shown to be a reasonable approximation for converters even with significantly varying emitter temperature distributions, except for possible transients involving thermionic burnout. 
This last point is by itself an important result. Individual diodes in a reactor core will probably not experience substantially nonuniform surface temperature deviations. However, several diodes are usually connected in series, as discussed in Chapter II, and even with power flattening, some axial power and temperature variations are inevitable. Thus, the simplifying assumption that all elements in any given stack have identical surface temperatures may in some instances lead to erroneous results. Possible extensions to multiple series-connected converter studies are discussed in Chapter VI. The following chapter describes some applications of the one diode reactor model to system start-up analysis. 


\title{
CHAPTER V
}

\author{
SIMULATED REACTOR SYSTEM START-UP
}

This chapter illustrates the use of the single thermionic diode reactor model as applied to two postulated start-up sequences. The method of start-up of an actual reactor containing in-core thermionic devices has as yet not been specified. However, there are currently two main approaches being considered in this area. These consist of maintaining constant diode output voltage or constant emitter temperature during start-up. Examples of both cases are investigated here.

\section{Reactor System Description}

The reactor model used is comparable to that discussed in Chapter II and illustrated in Figures 2.2 and 2.3. One exception is made in that the insulator and cladding are now collapsed into a single region with a composite describing equation.

During preliminary studies, it was found that one of the limiting factors in the speed of the integration routine was due to the large time constant associated with the electrical insulator region. Computer running time was reduced considerably when the insulator and cladding regions were combined. The collector could likewise have been collapsed into this region, but a more accurate description for the thermionic analysis and transient response is obtained with the collector separate. The loss of information and 
generality of the resulting model were found to be negligible when compared to the considerable improvement in computational time.

For the reactor model start-up cases considered here, the ratio of simulation time to computer time required was almost 10:1. Thus, a simulated start-up transient of 300 seconds duration required a corresponding computational time of approximately 30 seconds.

The reactor regions of the resulting single diode model include fuel, emitter, collector, insulator-cladding, and primary reactor coolant. No coolant transport delay or piping energy loss is assumed for the primary and secondary coolant loops. Thus, as described in Chapter II, the reactor coolant and primary-side heat exchanger equations may be collapsed into one with no loss of accuracy for this case of no coolant lag. Similarly, the secondaryside heat exchanger and bulk radiator equations are combined.

Each of these regions is characterized by its spatiallyaveraged temperature. For these simulations the materials used are: uranium-carbide fuel, tungsten emitter, molybdenum collector, aluminum dioxide insulator, niobium cladding, lithium coolant in both primary and secondary loops, and a stainless steel radiator.

The neutron dynamics are described by the prompt jump approximation. Reactivity feedbacks are included for the fuel, emitter, collector, insulator, and reactor coolant.

The surface and interelectrode gas parameters used are those specified by Wilkins (1968). In that report, Wilkins listed the 
appropriate data to calibrate the thermionic analysis to experimental current-voltage characteristics obtained for a polycrystalline heattreated tungsten emitter and a polycrystalline molybdenum collector.

\section{Start-up Cases}

The procedure used in analyzing the start-up cases was first to select a desired final system operating point. Steady-state calculations were then carried out for this full power equilibrium condition using equations comparable to those listed in Table 2.1 with the time derivatives set to zero.

The conditions chosen for this full power situation were an emitter temperature of $2000^{\circ} \mathrm{K}$, collector temperature of $1100^{\circ} \mathrm{K}$, diode output voltage of 0.7 volt, and a cesium reservoir temperature of $620^{\circ} \mathrm{K}$. The conditions for the thermionic analysis were thus prespecified so that a steady-state analysis could be conducted to determine the appropriate current and load resistance values to yield an output voltage of 0.7 volt. This consequently set the required emitter heat flux as well. This steady-state heat flux requirement was then used to size the radiator.

The resulting steady-state operating conditions and system parameters used are listed in Table 5.1. The indicated reactivity feedback coefficients correspond to a typical set used by Gronroos (1967). The dynamic equations and corresponding time constants are shown in Table 5.2 .

Although the scope of this study does not encompass general control system applications, the analysis of the two start-up cases 
TABLE 5.1

Full Power Equilibrium Conditions and System Parameters for Start-up Studies

\begin{tabular}{|c|c|c|c|c|c|c|c|c|}
\hline \multicolumn{9}{|c|}{ System Material Parameters } \\
\hline Item & Unit & $\begin{array}{l}\text { Fuel } \\
\text { UC }\end{array}$ & $\begin{array}{c}\text { Emitter } \\
W\end{array}$ & $\begin{array}{l}\text { Collector } \\
\text { Mo }\end{array}$ & $\begin{array}{c}\text { Insulator } \\
\mathrm{Al}_{2} \mathrm{O}_{3} \\
\end{array}$ & $\begin{array}{l}\text { Cladding } \\
\mathrm{Nb}\end{array}$ & $\begin{array}{r}\text { Coolant } \\
\mathrm{L} i-7\end{array}$ & $\begin{array}{l}\text { Radiator } \\
\text { SS }\end{array}$ \\
\hline$\overline{\mathrm{T}}_{i}$ & ${ }^{\circ} \mathrm{K}$ & 2123 & 2000 & 1100 & 1028 & 1028 & 988 & 962 \\
\hline$\rho_{0}$ & $\mathrm{gm} / \mathrm{cm}^{3}$ & 9.90 & 18.00 & 10.24 & 3.20 & 8.30 & 0.44 & 8.35 \\
\hline $\mathrm{c}_{\mathrm{P}}$ & $\mathrm{w}-\mathrm{s} / \mathrm{gm}-{ }^{\circ} \mathrm{K}$ & 0.268 & 0.188 & 0.294 & 1.19 & 0.31 & 4.14 & 0.837 \\
\hline $\mathrm{k}_{j}^{\mathrm{j}}$ & $\mathrm{w} / \mathrm{cm}-{ }^{\circ} \mathrm{K}$ & 0.230 & 1.590 & 1.160 & 0.0347 & 0.630 & & \\
\hline $\mathrm{R}_{j}$ & $\mathrm{~cm}$ & 1.50 & 1.60 & 1.725 & 1.775 & 1.850 & & 1.10 \\
\hline $\mathrm{L}_{i}$ & $\mathrm{~cm}$ & 5.0 & 5.0 & 5.0 & 5.0 & 6.0 & & 250.0 \\
\hline$\alpha_{j}^{j}$ & $\mathrm{~S} /{ }^{\circ} \mathrm{K}$ & $+4 \times 10^{-5}$ & $-8 \times 10^{-5}$ & $-1.5 \times 10^{-4}$ & 0 & $-1.5 \times 10^{-4}$ & $-1.5 \times 10^{-4}$ & 0 \\
\hline
\end{tabular}

\begin{tabular}{|c|c|c|c|c|c|c|c|c|c|c|c|}
\hline \multicolumn{6}{|c|}{ Thermionic Parameters } & \multicolumn{6}{|c|}{ Other Parameters } \\
\hline Item & Unit & Value & Item & Unit & Value & Item & Unit & Value & Item & Unit & Value \\
\hline $\mathrm{J}$ & $\mathrm{amp} / \mathrm{cm}^{2}$ & 19.8 & $\mathrm{~N}_{\mathrm{S}}$ & none & 5 & $\mathrm{n}_{0}$ & watt $/ \mathrm{cm}^{3}$ & 99 & $U_{H} A_{H}$ & watt $/{ }^{\circ} \mathrm{K}$ & 16200 \\
\hline $\mathrm{q}_{\mathrm{E}}^{\prime \prime}$ & watt $/ \mathrm{cm}^{2}$ & 69.6 & $\mathrm{~N}_{\mathrm{p}}$ & none & 30 & h & $\mathrm{w} / \mathrm{cm}^{2}-{ }^{\circ} \mathrm{K}$ & 6.0 & $N_{R P}$ & none & 50 \\
\hline $\mathrm{v}_{0}$ & volt & 0.7 & & & & $M_{p}$ & Kgm & 29.5 & $\varepsilon_{\mathrm{R}}$ & none & 0.8 \\
\hline $\mathrm{R}_{\mathrm{L}}$ & $\Omega-\mathrm{cm}^{2}$ & 0.035 & & & & $c_{p}$ & $\mathrm{w}-\mathrm{s} / \mathrm{gm}-{ }^{\circ} \mathrm{K}$ & 0.837 & $\lambda$ & $\sec ^{-1}$ & 0.1 \\
\hline $\mathrm{T}_{\mathrm{R}}$ & ${ }^{\circ} \mathrm{K}$ & 615 & & & & ${ }^{M_{S}}{ }^{P}$ & Kgm & 29.5 & $\beta$ & none & 0.0064 \\
\hline d & mil & 10 & & & & $\mathrm{C}_{\mathrm{P}}$ & $\mathrm{w}-\mathrm{s} / \mathrm{gm}-{ }^{\circ} \mathrm{K}$ & 0.837 & & & \\
\hline
\end{tabular}


TABLE 5.2.

Dynamic Equations for Start-up Studies

$$
n=\frac{.10}{1-\rho} ; \rho=\rho_{0}+\sum_{j} \alpha_{j}\left(\bar{T}_{j}-\bar{T}_{c o}\right)
$$

$\frac{d C}{d t}=n-0.1 C$

$\frac{d \bar{T}_{F}}{d t}=0.37 \cdot n-0.303\left(\bar{T}_{F}-\bar{T}_{E}\right)$

$\frac{d \bar{T}_{E}}{d t}=1.722\left(\bar{T}_{F}-\bar{T}_{E}\right)-3.050 q_{E}^{\prime \prime}$

$\frac{d \bar{T}_{C}}{d t}=3.172 q_{C}^{\prime \prime}-4.196\left(\bar{T}_{C}-\bar{T}_{I-C l}\right)$

$\frac{d \bar{T}_{I-C l}}{d t}=2.803\left(\bar{T}_{C}-\bar{T}_{I-C l}\right)-2.971\left(\bar{T}_{I-C l}-\bar{T}_{C O}\right)$

$\frac{d \bar{T}_{C O}}{d t}=0.390\left(\bar{T}_{I-C l}-\bar{T}_{C O}\right)-0.598\left(\bar{T}_{C O}-\bar{T}_{R}\right)$

$\frac{d \bar{T}_{R}}{1 t}=0.131\left(\bar{T}_{C O}-\bar{T}_{R}\right)-3.158 \times 10^{-12} \bar{T}_{R}^{4}$ 
investigated does necessitate the use of an approximate control scheme. The two cases of interest involve a constant diode voltage and a constant emitter temperature start-up respectively. In order to maintain these parameters to within some desired margin, changes in other system variables are required. The variable selected here for contro1 purposes is the load resistance.

For the cases of simulated start-up, the sequence proceeds as follows: a 1 cent step in reactivity is inserted. The reactor power undergoes an initial "prompt jump" and the system experiences a transient while adjusting to a new equilibrium. During the transient, the desired parameter (i.e., either diode voltage or emitter temperature) is sensed and the load resistance incremented appropriately if the parameter strays outside a prespecified tolerance level. Once the input reactivity is compensated by the inherent thermal feedback mechanisms, an additional 1 cent step is inserted. Thus, the start-up is a series of reactivity "bumps" until the system reaches the desired full power operating point. The two start-up cases investigated are discussed next.

\section{Constant Output Voltage Start-up}

The desire to maintain a constant diode output voltage arises from the electrical output requirements of the overall system. With respect to the required power conditioner which converts the reactor output electrical power to usable levels, it may be advantageous to operate the thermionic diodes at a constant voltage. 
For this example case, the reactor is assumed to be at an initial low power steady state with an emitter temperature of $1700^{\circ} \mathrm{K}$. This allowed the emitter temperature to remain within the region of validity of the thermionic analysis (as 1isted in Table 3.1) and still demonstrate system response for a sizeable change in operating conditions.

However, the appropriate collector temperature necessary to obtain a diode output voltage of 0.7 volt was below the region of applicability for the thermionic analysis. This resulted from the very low diode current and emitter heat flux values at the corresponding 0.7 volt point. In order to raise the collector temperature to a more reasonable value for thermionic calculations, the desired diode voltage condition of 0.7 volt was initially sacrificed. An initial collector temperature of $700^{\circ} \mathrm{K}$ could then be attained, and the resulting conditions for all important parameters are 1isted in Table 5.3.

The transient results of the simulated start-up are shown in Figure 5.1. Initial conditions for the temperature traces are indicated.

For the first three 1 cent steps in reactivity, no control is placed on the load resistance so that sufficient current would be drawn to maintain an elevated collector temperature. With the insertion of the fourth 1 cent step (at about 16 minutes), the load was slowly altered to bring the output voltage to 0.7 volt. The resulting increased resistance at first lowered the current and electron 
TABLE 5.3.

Initial Conditions for Constant Voltage Start-up

$$
\begin{aligned}
& \text { Parameter } \\
& \text { Value } \\
& \mathrm{n} \cdot . \quad . \quad . \quad . \quad . \quad 15.9 \text { watts } / \mathrm{cm}^{3} \\
& \overline{\mathrm{T}}_{\mathrm{F}} \quad \cdot \quad \cdot \quad \cdot \quad \cdot \quad \cdot \quad \cdot \quad \cdot 1720^{\circ} \mathrm{K} \\
& \overline{\mathrm{T}}_{\mathrm{E}} \cdot \cdot \quad \cdot \quad \cdot \quad \cdot \quad \cdot \quad \cdot \quad \cdot 1700^{\circ} \mathrm{K} \\
& \overrightarrow{\mathrm{T}}_{\mathrm{C}} \cdot \cdot \cdot \cdot \cdot \cdot \cdot \cdot \cdot 700^{\circ} \mathrm{K} \\
& \overline{\mathrm{T}}_{\mathrm{I}-\mathrm{C} 1} \quad \cdot \quad \cdot \quad \cdot \quad \cdot \quad \cdot 686^{\circ} \mathrm{K} \\
& \overline{\mathrm{T}}_{\mathrm{Co}} \cdot \cdot \cdot \cdot \cdot \cdot \cdot \cdot \cdot \cdot 678^{\circ} \mathrm{K} \\
& \overline{\mathrm{T}}_{\mathrm{R}} \quad \cdot \quad \cdot \quad \cdot \quad \cdot \quad \cdot \quad \cdot \quad \cdot 673^{\circ} \mathrm{K} \\
& \mathrm{q}_{\mathrm{E}}{ }^{\prime \prime} \cdot \text {. } . \quad . \quad . \quad .11 .2 \text { watts } / \mathrm{cm}^{2} \\
& \mathrm{~J} \quad . \quad . \quad . \quad . \quad . \quad .27 \mathrm{amps} / \mathrm{cm}^{2} \\
& \mathrm{v}_{\mathrm{o}} \cdot \cdot \cdot \cdot \cdot \cdot \cdot \cdot \quad \cdot \quad \cdot 0.21 \text { volts } \\
& \mathrm{R}_{\mathrm{L}} \quad \cdot \quad \cdot \quad \cdot \quad \cdot \quad \cdot \quad \cdot 0.094 \Omega-\mathrm{cm}^{2} \\
& \mathrm{~T}_{\mathrm{R}} \quad \cdot \quad \cdot \quad \cdot \quad \cdot \quad \cdot \quad \cdot 620^{\circ} \mathrm{K} \\
& \text { d . . . . . . } 10 \mathrm{mils}
\end{aligned}
$$



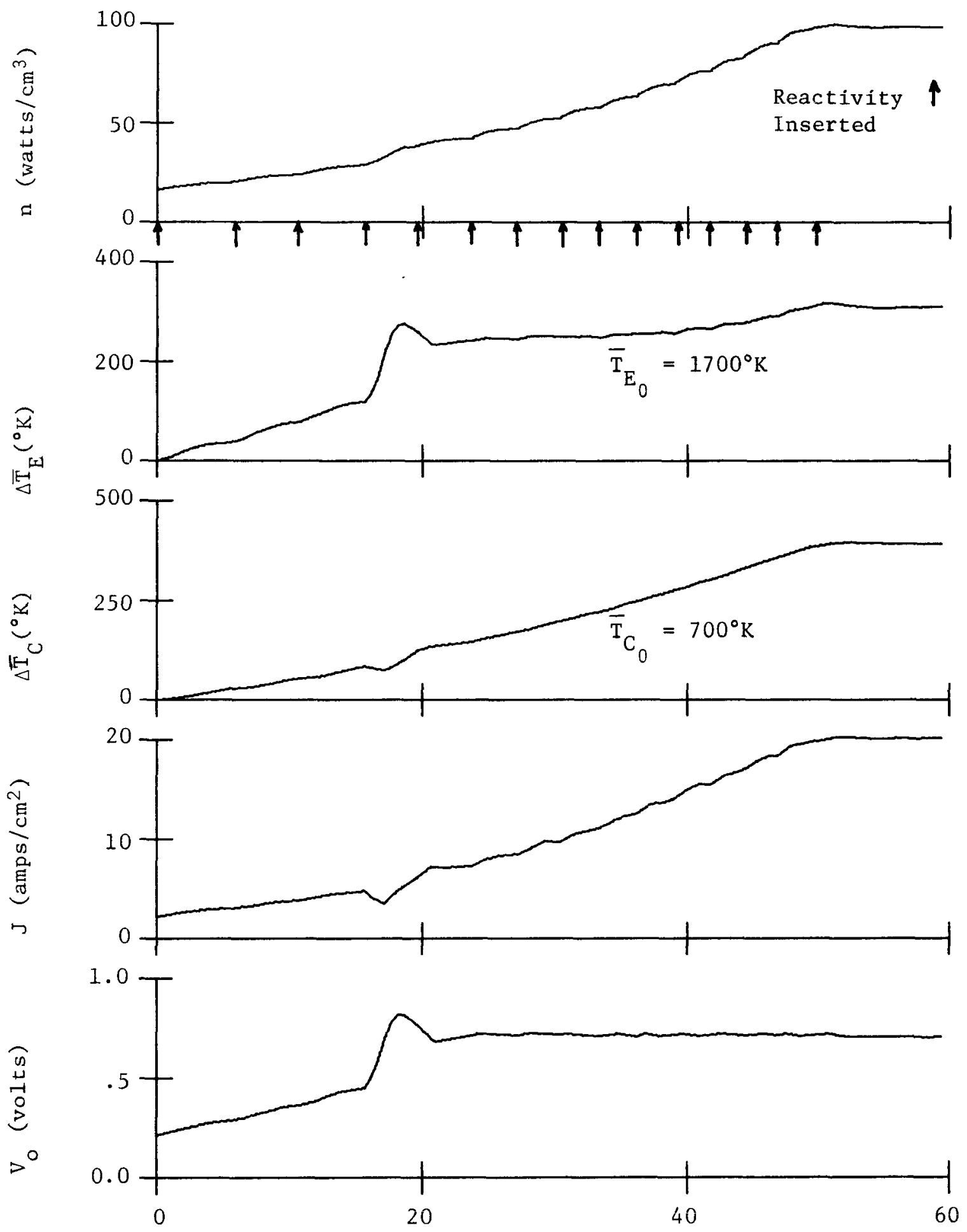

Time (minutes)

Figure 5.1. Constant Voltage Start-up 
cooling, thus rapidly raising the emitter temperature and lowering the collector.

As the voltage was finally brought to 0.7 volt and maintained within \pm 0.02 volt of that point, the current recovered and began increasing. For this particular set of circumstances, the subsequent rate of current increase was sufficient to maintain the emitter at about $1950^{\circ} \mathrm{K}$ for a substantial period of time while the collector temperature slowly rose. A total input of 15 cents in reactivity was adequate to bring the reactor system to its final operating point after almost one hour.

Constant Emitter Temperature Start-up

Interest in maintaining a constant emitter temperature stems from the desire to avoid thermal cycling within the emitter region. Large and sudden changes in the emitter surface temperature may lead to the formation of cracks or distortions in the emitter. These might result in a diode short circuit or produce adverse or unpredictable effects on performance. Also, by maintaining a constant emitter temperature, the possibility of thermionic burnout, as discussed in Chapter IV, may be minimized.

For this case, the reactor power was assumed to have been brought to a level where the emitter temperature was at its desired full power value of $2000^{\circ} \mathrm{K}$. The converter was assumed to be at open circuit so that no electron cooling was present. At this emitter temperature, radiation cooling and cesium conduction across the diode gap were sufficient to produce an initial collector temperature of 
almost $700^{\circ} \mathrm{K}$. The various initial conditions for important parameters are listed in Table 5.4. Transient results are shown in Figure 5.2.

When the initial $1 c$ step in reactivity was inserted, the diode electrical circuit was assumed to be simultaneously phased in at a high load resistance value. Because the current was low, the emitter was not substantially cooled initially. The corresponding output voltage was almost 2 volts.

During this and all subsequent insertions, the load resistance was continually incremented to maintain the emitter temperature within a $\pm 10^{\circ} \mathrm{K}$ band about the $2000^{\circ} \mathrm{K}$ point. The emitter oscillations were rapid and pronounced at first due to the large resistance increments required during the initial stages of the start-up. As the full power operating point was approached, the necessary load changes to maintain the desired emitter temperature became substantially smaller.

As seen in Figure 5.2., the emitter temperature remained in the 0 to $+10^{\circ} \mathrm{K}$ band for the majority of the start-up transient. Since the thermal power was increasing, the emitter temperature also rose. Each time it exceeded $2010^{\circ} \mathrm{K}$, a load resistance decrease resulted, which increased the current and electron cooling to trim the emitter temperature back toward $2000^{\circ} \mathrm{K}$. However, the continually rising input power soon caused the emitter temperature to increase again.

The collector temperature rose very slowly at first due to the large heat sink resulting from the fixed radiator size designed to accommodate the full power heat rejection requirements. The collector 
TABLE 5.4 .

Initial Conditions for Constant Emitter Temperature Start-up

$$
\begin{aligned}
& \text { Parameter } \\
& \text { Value } \\
& \mathrm{n} \quad . \quad . \quad . \quad . \quad . \quad .15 .1 \text { watts } / \mathrm{cm}^{3} \\
& \overline{\mathrm{T}}_{\mathrm{F}} \cdot \cdot \quad \cdot \quad \cdot \quad \cdot \quad \cdot \quad \cdot \quad \cdot 2019^{\circ} \mathrm{K} \\
& \overline{\mathrm{T}}_{\mathrm{E}} \cdot \cdot \cdot \cdot \cdot \cdot \cdot \cdot 2000^{\circ} \mathrm{K} \\
& \overline{\mathrm{T}}_{\mathrm{C}} \cdot \cdot \cdot \cdot \cdot \cdot \cdot 698^{\circ} \mathrm{K} \\
& \overline{\mathrm{T}}_{\mathrm{I}-\mathrm{Cl} 1} \cdot \cdot \cdot \cdot \cdot \cdot \cdot \cdot 684^{\circ} \mathrm{K} \\
& \overline{\mathrm{T}}_{\mathrm{Co}} \cdot \quad \cdot \quad \cdot \quad \cdot \quad \cdot \quad \cdot 677^{\circ} \mathrm{K} \\
& \overline{\mathrm{T}}_{\mathrm{R}} \cdot \cdot \quad \cdot \quad \cdot \quad \cdot \quad \cdot \quad \cdot \quad \cdot 672^{\circ} \mathrm{K} \\
& \mathrm{q}_{\mathrm{E}}{ }^{\prime \prime} \quad \cdot \quad \cdot \quad \cdot \quad \cdot \quad \cdot \quad \cdot 10.6 \text { watts } / \mathrm{cm}^{2}
\end{aligned}
$$

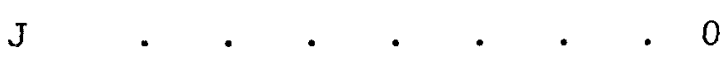

$$
\begin{aligned}
& \mathrm{V}_{\mathrm{o}} \cdot \cdot \quad \cdot \quad \cdot \quad \cdot \quad \cdot \quad \cdot \quad \cdot \text { open circuit } \\
& \mathrm{R}_{\mathrm{L}} \cdot \cdot \quad \cdot \quad \cdot \quad \cdot \quad \cdot \quad \text { infinite } \\
& \mathrm{T}_{\mathrm{R}} \quad \cdot \quad \cdot \quad \cdot \quad \cdot \quad \cdot \quad \cdot \quad \cdot 620^{\circ} \mathrm{K} \\
& \mathrm{d} . \quad . \quad . \quad . \quad 10 \mathrm{mils}
\end{aligned}
$$



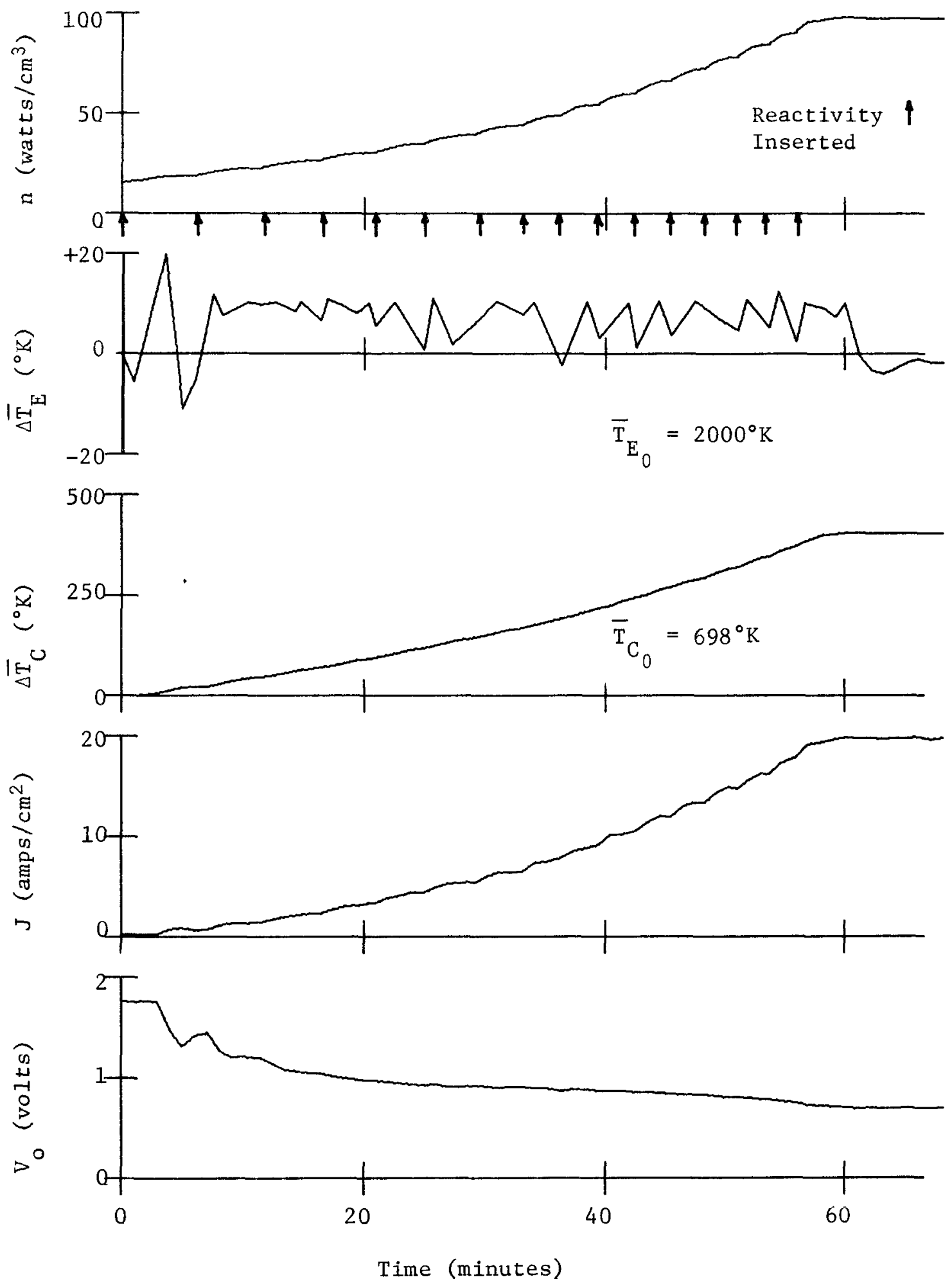

Figure 5.2. Constant Emitter Temperature Start-up 
temperature and other system variables slowly increased in a generally smooth manner to the full power equilibrium level. The simulated start-up for this particular case required the insertion of $16 \mathrm{c}$ worth of reactivity during a period of over one hour.

\section{Discussion of Results}

While this method of control by load resistance used for these example start-up cases may or may not be utilized in an actual thermionic system, the results obtained are encouraging and instructive. The earlier contention that thermionic reactors should be relatively easy to control is supported by the results shown here. Indeed, these two cases demonstrate that very reasonable responses during start-up may be obtained even with the crude control scheme used here. They indicate that this slow responding thermionic reactor system may not require a fast dynamically reacting controller to insure stable operation.

The refinement of incorporating other possible control schemes could be an important extension of this work. For example, if the thermal stresses in the emitter for the case depicted in Figure 5.2 are too severe, the magnitude of the emitter fluctuations could be reduced through use of a refined control system or possibly simply smaller reactivity insertions. If the overshoot on voltage, as shown in Figure 5.1 when the load control is initiated, is not satisfactorily damped for a specific application, the same type of analysis just mentioned could be utilized. Also, the "bumps" in the diode current 
response, as shown in both Figures 5.1 and 5.2 , could be smoothed out by a more refined control method.

These "bumps" in diode current are a direct result of the power surges following the stepwise reactivity insertions. As the start-up progresses, the time between reactivity insertions continually decreases. This situation results from the more pronounced initial jumps in the reactor power density as the magnitude of the power level increases. Consequently, the required compensating reactivity feedback from the various thermal regions is achieved quicker. The difference of $1 c$ in total reactivity insertion between the two startaup cases arises from the different initial conditions present for each case.

Thus, meaningful applications employing the single diode reactor model have been demonstrated. Constructive insight into the actual dynamic system behavior during start-up or other situations involving major changes in operating conditions may be obtained. An important result demonstrated by these simulated start-up cases was to indicate that even simple control methods seem adequate to produce smooth and orderly system transitions during transients over wide ranges of reactor operations. 
CHAPTER VI

CONCLUSIONS

A reasonably detailed single diode model to investigate the dynamic behavior of a nuclear reactor containing in-core thermionic devices has been formulated. The resulting representation is applicable to situations involving substantial changes in system operating conditions as would be experienced during start-up transients. The validity of both the modeling of the system describing equations and the converter physics analysis are tested over wide ranges of anticipated reactor operation.

It is demonstrated that the solution of the nonlinear system transient equations by numerical techniques on a digital computer is indeed accurate and possibly better than comparable analog computer simulations. The computational running times for transient studies are shown to be reasonable. The application of the overall digital computer routine is quite versatile and may be adapted to represent a variety of desired system representations by merely specifying the proper transient equations and appropriate system parameters.

The response of the proposed analytical converter physics model is proven to be valid over wide ranges of actual diode operations. The results are accurate for many types of anticipated thermionic perturbations. Comparison studies conducted here further indicate that this more complex description for the thermionic 
processes involved is indeed required to obtain realistic values for the diode characteristics during large changes in operating conditions. Use of the method is quite general and applications to any typical diode representation are possible by simply supplying the appropriate input data.

The resulting single diode thermionic reactor model may be employed to obtain insight into the overall system dynamic behavior during large changes in the system operating conditions, as for example during the start-up sequence. Utilization of this method to include more refined external controls or to study such aspects as coolant transport delay effects is a logical extension of this work. The use of spatial-averaging techniques to describe dynamic response is demonstrated to be generally acceptable. One exception concerns cases involving thermionic burnout for diodes with substantially varying axial emitter temperature distributions. This is an important finding in that the actual temperature distributions in a thermionic reactor core will indeed be varying axially to some extent. Thus, the assumption that all diodes in the reactor may be represented by an equal and uniform emitter temperature could possibly lead to erroneous results in some situations.

The analysis of the single diode representation developed in this work may be extended to investigate the dynamic response of a stack of series-connected diodes such as would be present in an actual thermionic reactor core. Applications of a simplified multiple parallel-connected diode treatment have been shown to be feasible 
during this study. The comparable thermionic iterative condition for a series-connected stack would be

$$
\sum_{j} V_{o_{j}}=J \cdot R_{L}
$$

where the summation combines the resulting voltages for each converter in the stack. With the specification of the appropriate axiallyvarying thermal describing equations, transient studies employing this more realistic system model could be conducted. 
APPENDIX

SYSTEM DYNAMICS PROGRAM

The Appendix is omitted in this copy. 


\section{LIST OF REFERENCES}

Abramowitz, A., and Stegun, I. A., Handbook of Mathematical Functions, New York: Dover Publications, 1965.

Angrist, S. W., Direct Energy Conversion, Boston: Allyn and Bacon, Inc., 1965.

Brehm, R. L., Hetrick, D. L., and Schmidt, T. R., "Stability Study of In-core Reactor Concepts," Nuclear Applications and Technology, I, 117, August 1969.

Chapman, S., and Cowling, T. G., The Mathematical Theory of NonUniform Gases, London: Cambridge University Press, 1961.

Gronroos, H. G., "Analog Studies of Thermionic Reactor Dynamics," JPL SPS 37-45, Vol. IV, pp. 136-162 (1967).

Gronroos, H. G., and Davis, J. P., "Stability and Control Considerations for Thermionic Reactors," Proceedings of the Symposium on Thermionic Electrical Power Generation, Stressa, Italy, May 1968 .

Hansen, L. K., "Ion Current Effect in Cesium Diodes," Proceedings of the 25 th Annual Phys. Elec. Conference, Cambridge, Mass., March $1965 a$,

Hansen, L. K., "Some Effects of Ion Generation and Transport Processes on Ignited Mode Diode Parameters," Proceedings of the Thermionic Conversion Specialist Conference, San Diego, Calif., October $1965 \mathrm{~b}$.

Hansen, L. K., "Ion-Current and Schottky Effects in Thermionic Diodes," J. Applied Phys., 38, 4345 (1967).

Hansen, L. K., and Warner, C., "Transport Effects in the Unignited Mode of Thermionic Diodes," Proceedings of the 23rd Annual Phys. Elec. Conference, Cambridge, Mass., March 1963a.

Hansen, L. K., and Warner, C., "The Electron-Rich, Unignited Mode of Thermionic Converters," Proceedings of the Thermionic Conversion Specialist Conference. Gatlinburg, Tenn., October 1963b. 
Hansen, L. K., and Warner, C., "Uni.gnited and Ignited Mode Characteristics of a Guard Ring Converter," Proceedings of the Thermionic Conversion Specialist Conference, Cleveland, o., October 1964.

Hetrick, D. L., Dynamics of Nuclear Reactors, Chicago: University of Chicago Press, 1970.

Kitrilakis, S., and Meeker, M., "Experimental Determination of the Heat Conduction of Cesium Gas," J. Adv. Energy Conversion, $\underline{3}$ 59 (1963).

Kohl, W. H., Handbook of Materials and Techniques for Vacuum Devices, New York: Reinhold, 1967.

Krogh, F. T., "Variable Order Integrators for the Numerical Solution of Ordinary Differential Equations," JPL Sec. 314 (to be published), 1969.

Landrot, J. P., Bliaux, J., and List, D., "Analog Studies of a Nuclear Heated Thermionic Converter," Proceedings of the Symposium on Thermionic Electrical Power Generation, London, September 1965.

Levine, J. D., and Gyftopoulos, E. P., "Adsorption Physics of Metallic Particles," Surface Science, 1, 171 (1964).

Lewis, H. R., and Stovall, E. J., "Fortran Version of Nordsieck's Scheme for Numerical Integration of Differential Equations," LA-3292, March 1965.

Peelgren, M., Gronroos, H. G., Davis, J. P., and Ernst, D., "Thermionic Diode Kinetics Experiment - Design and Initial Operation," Proceedings of the Thermionic Conversion Specialist Conference, Carmel, Calif., October 1969.

Rasor, N. S., "Analytical Description of Cesium Diode Phenomenology," Proceedings of the Symposium on Thermionic Electrical Power Generation, London, September 1965.

Rasor, N. S., and Warner, C., "Correlation of Emission Processes for Adsorbed Alkali Films on Metal Surfaces," J. Applied Phys., 35, 2589 (1964).

Schock, A., "Effect of Cesium Pressure on Thermionic Stability," Proceedings of the Symposium on Thermionic Electrical Power Generation, Stressa, Italy, May 1968. 
Shavit, A., and Hatsopoulos, G. N., "Operation of a Thermionic Converter for the Ion-Rich Unignited Mode," Proceedings of the Thermionic Conversion Specialist Conference, Cleveland, 0., October 1964 .

Steiner, D., and Gyftopoulos, E. P., "An Equation for the Prediction of Bare Work Functions," Proceedings of the 27 th Annual Phys. Elec. Conference, Cambridge, Mass., March 1967.

Warner, C., "Theory of the Ignited Mode with Electron Temperature Variations $\mathrm{T}(\mathrm{x})$," Proceedings of the Thermionic Conversion Specialist Conference, San Diego, Calif., October 1965.

Weaver, L. E., Gronroos, H. G., Guppy, J. G., and Davis, J. P., "A Control Study for an In-Core Thermionic Reactor," JPL TR 321355, January 1969.

Wilkins, D. R., "SIMCON - A Digital Computer Program for Computing Thermionic Converter Performance Characteristics," GESR-2109, February 1968 .

Wilkins, D. R., and Gyftopoulos, E. P., "Thermionic Converters Operating in the Ignited Mode, Part I: Theoretical Output Current Characteristics, Part II: A Quasi-Equilibrium Model for the Interelectrode Plasma," J. Applied Phys., 37, 2888 (1966a).

Wilkins, D. R., and Gyftopoulos, E. P., "Transport Phenomena in LowEnergy Plasmas," J. Applied Phys., 37, 3533 (1966b).

Wilkins, D. R., and Gyftopoulos, E. P., "Theory of Thermionic Converter Extinguished-Mode Operation with Applications to Converter Diagnostics," J. Applied Phys., 38, 12 (1967).

Wilkins, D. R., and McCandless, R. J., "Thermionic Converter Plasma AnaLysis, Interim Report," GESP-9004, February 1969. 